\title{
Decarboxylative N-Alkylation of Azoles Through Visible-Light-Mediated Organophotoredox Catalysis
}

Rino Kobayashi, ${ }^{\dagger}$ Shotaro Shibutani, ${ }^{\dagger}$ Kazunori Nagao, ${ }^{* \dagger}$ Zenichi Ikeda, ${ }^{\ddagger}$ Junsi Wang, Ignacio Ibáñez, ${ }^{\ddagger}$ Matthew Reynolds, ${ }^{\ddagger}$ Yusuke Sasaki, ${ }^{\star}$ and Hirohisa Ohmiya ${ }^{* \dagger}{ }^{\S}$

†Division of Pharmaceutical Sciences, Graduate School of Medical Sciences, Kanazawa University, Kakuma-machi, Kanazawa 920-1192, Japan

\$esearch, Takeda Pharmaceutical Company Limited,

2-26-1, Muraoka-Higashi, Fujisawa, Kanagawa 251-8555, Japan

§JST, PRESTO, 4-1-8 Honcho, Kawaguchi, Saitama 332-0012, Japan

*E-mail: Kazunori Nagao: nkazunori@p.kanazawa-u.ac.jp

Hirohisa Ohmiya: ohmiya@p.kanazawa-u.ac.jp

\section{Table of Contents}

\section{- Supplementary Methods}

1. Instrumentation and Chemicals

2. Synthesis of Precursur of Protein Degrader-like Molecule (2q)

3. Characterization Data for Redox Active Ester

4. Preparation of Collidine $\cdot \mathrm{HBF}_{4}$

5. Procedures for Decarboxyaltive N-Alkylation of Azoles

S5-S6

6. Characterization Data for N-Alkylated Azoles

7. Results with Other Class of Photoredox Catalysts

S17

8. Effect of Collidine $\cdot \mathrm{HBF}_{4}$

9. Cyclic Voltammetry (CV) Experiments

S18-S19

10. UV-Vis Spectra of Reaction Mixtures 


\section{- Supplementary Methods}

\section{Instrumentation and Chemicals}

NMR spectra were recorded on a Bruker AVANCE NEO 400N spectrometer, operating at 400 $\mathrm{MHz}$ for ${ }^{1} \mathrm{H} \mathrm{NMR}, 100.6 \mathrm{MHz}$ for ${ }^{13} \mathrm{C} \mathrm{NMR}$ and Bruker Ultra Shield-300 (300 MHz), operating at ng at $300 \mathrm{MHz}$ for ${ }^{1} \mathrm{H} \mathrm{NMR}, 75 \mathrm{MHz}$ for ${ }^{13} \mathrm{C}$ NMR. Chemical shift values for ${ }^{1} \mathrm{H}$ and ${ }^{13} \mathrm{C}$ are referenced to $\mathrm{Me}_{4} \mathrm{Si}$ and the residual solvent resonances, respectively. Chemical shifts were reported in $\delta$ ppm. Mass spectra were obtained with JMS-T100TD (DART and ESI-TOF). TLC analyses were performed on commercial glass plates bearing 0.25-mm layer of Merck Silica gel $60 \mathrm{~F}_{254}$. Silica gel (Wakosil ${ }^{\circledR}$ 60, 64 210 $\mu \mathrm{m}$ ) was used for column chromatography. Biotage Selekt was used for purification. IR spectra were measured with a Thermo Scientific iD7 ATR Accessory for the Thermo Scientific Nicolet iS5 FT-IR Spectrometer. Melting points were measured on a Stanford Research Systems MPA100. CV measurements were recorded with a Hokuto Denko HZ-7000 potentiostat. UV-Vis absorption spectra were recorded on a Shimadzu UV-1900. Fluorescence spectra were recorded on a Shimadzu RF-6000. Kessil A160W Tuna Blue (highest blue and intensity setting) was used as a light source. TEKNOS MG9 was used as a fan.

All reactions were carried out under nitrogen atmosphere. Materials were obtained from commercial suppliers or prepared according to standard procedures unless otherwise noted. $\mathrm{LiBF}_{4}$ 1,2-dichloroethane and 1,2-dichlorobenzene were purchased from Sigma-Aldrich Japan., and used as received. MeCN, THF and DCM were purchased from FUJIFILM Wako Pure Chemical Co., stored under nitrogen, and used as received.

Azoles 1a-c and 1g-l were purchased from Tokyo Chemical Industry Co., stored under nitrogen, and used as received. Azoles 1d and 1f were purchased from BLD Pharmatech Ltd., stored under nitrogen, and used as received. Azoles $1 \mathbf{e}$ and $\mathbf{1 i}$ were prepared by protection of the corresponding amine, respectively. Redox active esters 2 were prepared by prepared by the reported procedure. ${ }^{1}$ PTH1-5 were prepared by the reported procedure..$^{2-4}$ A1-3 were prepared by the reported procedure. ${ }^{5}$ 
2. Synthesis of Precursur of Protein Degrader-like Molecule (2q)<smiles>CCCCOC(=O)C(C)C</smiles>

S1

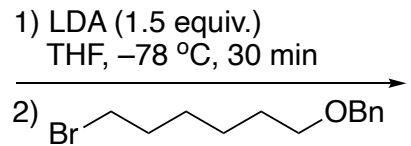

THF, $-78^{\circ} \mathrm{C}$ to rt. o.n.

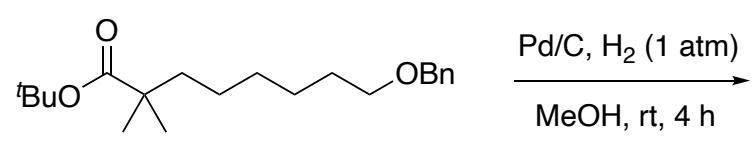

S2, $47 \%$<smiles>CCCCOC(=O)C(C)(C)CCCCCCO</smiles>

S3, $95 \%$

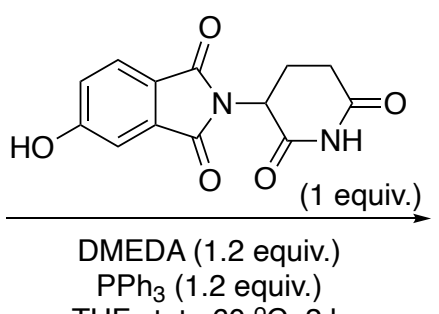

THF, rt. to $60^{\circ} \mathrm{C}, 2 \mathrm{~h}$<smiles>CC(C)(C)OC(=O)C(C)(C)CCCCCCOc1ccc2c(c1)C(=O)N(C1CCC(=O)NC1=O)C2=O</smiles>

1) TFA, rt, $1 \mathrm{~h}$

2) NHPI (1.5 equiv.), DIC (1.4 equiv.) DMAP (5 mol \%), THF, rt, o.n.

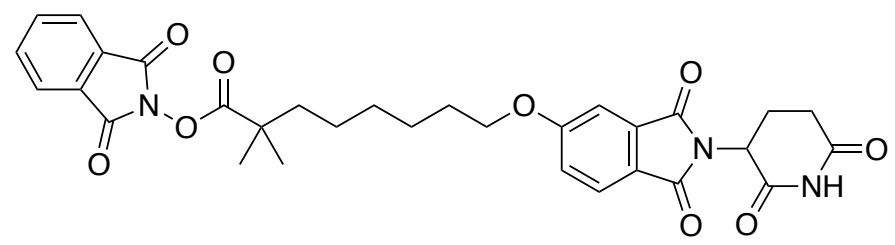

2q, $65 \%$ (in 2 steps)

Figure S1. Synthesis of 2q.

Synthesis of S2. To a solution of lithium diisopropylamide (10.4 ml, $10.4 \mathrm{mmol})(1 \mathrm{M})$ in THF (20 $\mathrm{mL})$ at $-78{ }^{\circ} \mathrm{C}$ was added tert-butyl isobutyrate $\mathbf{S 1}(1 \mathrm{~g}, 6.93 \mathrm{mmol})$ in THF $(5 \mathrm{~mL})$ dropwise. The mixture was stirred at $-78{ }^{\circ} \mathrm{C}$ under Ar for $30 \mathrm{~min}$. $\{[(6$-bromohexyl)oxy $]$ methyl $\}$ benzene $(2.02 \mathrm{ml}$, $9.01 \mathrm{mmol})$ in THF $(2 \mathrm{~mL})$ was added dropwise to the solution at $-78^{\circ} \mathrm{C}$. The mixture was allowed to warm to room temperature overnight. The mixture was quenched with sat. $\mathrm{NH}_{4} \mathrm{Cl}$ aq. and extracted with EtOAc. The organic layer was separated, washed with brine, dried over $\mathrm{MgSO}_{4}$, filtered and concentrated in vacuo. The residue was purified by column chromatography on silica gel (100:0-93:7, hexane/EtOAc) to give tert-butyl 8-(benzyloxy)-2,2-dimethyloctanoate S2 (1.090 g, $3.26 \mathrm{mmol}$, $47.0 \%$ ) as a colorless oil. IR (neat) 697, 735, 853, 1142, 1366, 1474, 1722, 2858, 2934, $2975 \mathrm{~cm}^{-1}$. ${ }^{1} \mathbf{H}$ NMR $\left(300 \mathrm{MHz}, \mathrm{CDCl}_{3}\right) \delta 7.36-7.26(\mathrm{~m}, 5 \mathrm{H}), 4.53-4.44(\mathrm{~m}, 2 \mathrm{H}), 3.46(\mathrm{t}, J=6.6 \mathrm{~Hz}, 2 \mathrm{H}), 1.68-$ $1.54(\mathrm{~m}, 2 \mathrm{H}), 1.50-1.40(\mathrm{~m}, 11 \mathrm{H}), 1.40-1.18(\mathrm{~m}, 6 \mathrm{H}), 1.10(\mathrm{~s}, 6 \mathrm{H}) .{ }^{13} \mathbf{C ~ N M R}\left(75 \mathrm{MHz}, \mathrm{CDCl}_{3}\right) \delta$ $177.4,138.7,128.3(\times 2 \mathrm{C}), 127.6(\times 2 \mathrm{C}), 127.5,79.5,72.9,70.5,42.6,40.7,30.0,29.7,28.0(\times 3 \mathrm{C})$, 26.1, $25.2(\times 2 \mathrm{C}), 24.9$. HRMS-DART $(\mathrm{m} / \mathrm{z}):\left[\mathrm{M}+\mathrm{NH}_{4}\right]^{+}$calcd for $\mathrm{C}_{21} \mathrm{H}_{38} \mathrm{NO}_{3}{ }^{+}, 352.2846$; found, 352.2846 .

Synthesis of S3. Pd/C (347 mg, $0.33 \mathrm{mmol})$ was added to a solution of S2 (1.09 g, $3.26 \mathrm{mmol})$ in $\mathrm{MeOH}(30 \mathrm{ml})$ at room temperature under Ar. The mixture was stirred at room temperature under $\mathrm{H}_{2}$ for $4 \mathrm{~h}$. The mixture was filtered and concentrated to give tert-butyl 8-hydroxy-2,2-dimethyloctanoate S3 (758 mg, $3.10 \mathrm{mmol}, 95 \%$ ) as a colorless oil. IR (neat) 852, 1057, 1147, 1255, 1367, 1390, 1474, 1723, 2860, 2934, 2975, $3380 \mathrm{~cm}^{-1} .{ }^{1} \mathbf{H}$ NMR (300 MHz, $\left.\mathrm{CDCl}_{3}\right) \delta 3.70-3.59$ (m, $\left.2 \mathrm{H}\right) .1 .61-1.45$ $(\mathrm{m}, 4 \mathrm{H}), 1.43$ (s, $9 \mathrm{H}), 1.40-1.23(\mathrm{~m}, 5 \mathrm{H}), 1.20$ (m, $1 \mathrm{H}), 1.10(\mathrm{~s}, 6 \mathrm{H}) .{ }^{13} \mathbf{C}$ NMR $\left(75 \mathrm{MHz}, \mathrm{CDCl}_{3}\right)$ $\delta 177.4,79.6,63.1,42.7,40.7,32.8,30.0,28.1(\times 3 \mathrm{C}), 25.6,25.2(\times 2 \mathrm{C}), 24.9$. HRMS-DART $(\mathrm{m} / \mathrm{z})$ : $\left[\mathrm{M}+\mathrm{NH}_{4}\right]^{+}$calcd for $\mathrm{C}_{14} \mathrm{H}_{32} \mathrm{NO}_{3}{ }^{+}, 262.2377$; found, 262.2378 . 
Synthesis of S4. Bis(2-methoxyethyl) (E)-diazene-1,2-dicarboxylate (DMEDA) (460 mg, 1.96 $\mathrm{mmol}$ ) was added to a solution of $\mathbf{S 3}$ (400 mg, $1.64 \mathrm{mmol})$, triphenylphosphane (515 mg, $1.96 \mathrm{mmol})$, 2-(2,6-dioxopiperidin-3-yl)-5-hydroxyisoindoline-1,3-dione (449 mg, $1.64 \mathrm{mmol})$ in THF (10 ml) at room temperature. The mixture was stirred at room temperature overnight. After being stirrd at $60{ }^{\circ} \mathrm{C}$ for $2 \mathrm{~h}$, the mixture was concentrated in vacuo. The residue was purified by column chromatography on silica gel (95:5-50:50, hexane/EtOAc) to give tert-butyl 8-\{[2-(2,6-dioxopiperidin-3-yl)-1,3dioxoisoindolin-5-yl]oxy \}-2,2-dimethyloctanoate $\mathbf{S 4}(315 \mathrm{mg}, 0.629 \mathrm{mmol}, 38.4 \%)$ as a colorless amorphous solid. IR (neat) 749, 1143, 1199, 1257, 1289, 1387, 1617, 1713, 1774, $2938 \mathrm{~cm}^{-1} .{ }^{1} \mathbf{H}$ NMR $\left(300 \mathrm{MHz}, \mathrm{CDCl}_{3}\right) \delta 8.15(\mathrm{~s}, 1 \mathrm{H}), 7.77(\mathrm{~d}, J=8.4 \mathrm{~Hz}, 1 \mathrm{H}), 7.33(\mathrm{~d}, J=2.1 \mathrm{~Hz}, 1 \mathrm{H}), 7.18(\mathrm{dd}$, $J=8.4,2.1 \mathrm{~Hz}, 1 \mathrm{H}), 4.96(\mathrm{dd}, J=12.0,5.4 \mathrm{~Hz}, 1 \mathrm{H}), 4.11-4.04(\mathrm{~m}, 2 \mathrm{H}), 2.95-2.72(\mathrm{~m}, 3 \mathrm{H}), 2.14(\mathrm{~m}$, 1H), 1.88-1.77 (m, 2 H), 1.52-1.45 (m, 4 H), 1.45-1.41 (m, 9 H), 1.39-1.27 (m, 4 H), 1.12 (s, $6 \mathrm{H})$. ${ }^{13}$ C NMR $\left(75 \mathrm{MHz}, \mathrm{CDCl}_{3}\right) \delta 177.3,171.0,168.1,167.2,167.0,164.6,134.3,125.5,123.3,120.7$, 108.9, 79.6, 69.1, 49.3, 42.6, 40.6, 31.4, 29.8, 28.9, $28.02(\times 3 \mathrm{C}), 25.8,25.2(\times 2 \mathrm{C}), 24.8,22.7$. HRMS-DART $(\mathrm{m} / \mathrm{z})$ : $\left[\mathrm{M}+\mathrm{NH}_{4}\right]^{+}$calcd for $\mathrm{C}_{27} \mathrm{H}_{40} \mathrm{~N}_{3} \mathrm{O}_{7}{ }^{+}, 518.2861$; found, 518.2859.

Synthesis of 2q. The mixture of $\mathbf{S 4}(300 \mathrm{mg}, 0.60 \mathrm{mmol})$ and TFA (3 mL, $38.9 \mathrm{mmol})$ was stirred at room temperature for 1 hour. The mixture was concentrated in vacuo to give 8-\{[2-(2,6dioxopiperidin-3-yl)-1,3-dioxoisoindolin-5-yl]oxy\}-2,2-dimethyloctanoic acid (246 mg, $0.55 \mathrm{mmol}$, $92 \%)$ as a dark yellow oil. This product was subjected to the next reaction without further purification. ${ }^{1} \mathbf{H}$ NMR $\left(300 \mathrm{MHz}, \mathrm{CDCl}_{3}\right) \delta 8.35(\mathrm{~m}, 1 \mathrm{H}), 7.77(\mathrm{~d}, J=8.40 \mathrm{~Hz}, 1 \mathrm{H}), 7.34(\mathrm{~m}, 1 \mathrm{H}), 7.18(\mathrm{~m}, 1 \mathrm{H})$, $4.96(\mathrm{dd}, J=12.0,5.4 \mathrm{~Hz}, 1 \mathrm{H}), 4.08(\mathrm{~s}, 2 \mathrm{H}), 2.96-2.70(\mathrm{~m}, 4 \mathrm{H}), 2.22-2.10(\mathrm{~m}, 1 \mathrm{H}), 1.90-1.75(\mathrm{~m}$, $2 \mathrm{H}), 1.61-1.26(\mathrm{~m}, 9 \mathrm{H}), 1.26-1.14(\mathrm{~m}, 6 \mathrm{H})$.

To a solution of 8-\{[2-(2,6-dioxopiperidin-3-yl)-1,3-dioxoisoindolin-5-yl] oxy $\}-2,2-$ dimethyloctanoic acid (246 mg, $0.55 \mathrm{mmol}$ ) and isoindole-1,3-dione (NHPI) (135 mg, $0.83 \mathrm{mmol})$ in THF (10 ml) was added DMAP (3.38 mg, $0.03 \mathrm{mmol})$ and N,N'-Diisopropylcarbodiimide (DIC) $(0.119 \mathrm{ml}, 0.77 \mathrm{mmol})$ and the reaction mixture was stirred overnight at room temperature. The mixture was poured into water and extracted with EtOAc. The organic layer was separated, washed with water and brine, dried over $\mathrm{MgSO} 4$ and concentrated in vacuo. The residue was purified by column chromatography on silica gel (100:0-80:20, DCM/EtOAc) to give 1,3-dioxoisoindolin-2-yl 8-\{[2-(2,6-dioxopiperidin-3-yl)-1,3-dioxoisoindolin-5-yl] oxy $\}$-2,2-dimethyloctanoate $2 \mathbf{q}$ (253 mg, $0.429 \mathrm{mmol}, 78 \%$ ) as an off-white amorphous solid. IR (neat) 698, 750, 1050, 1199, 1256, 1289, 1382, 1615, 1713, 1742, 1779, 2968, $3338 \mathrm{~cm}^{-1}$. ${ }^{\mathbf{1}} \mathbf{H}$ NMR (400 MHz, CDCl 3$) \delta 7.93-7.76(\mathrm{~m}, 6 \mathrm{H})$, 7.35 (s, 1H), 7.19 (d, $J=8.4 \mathrm{~Hz}, 1 \mathrm{H}), 4.96(\mathrm{dd}, J=10.8,4.8 \mathrm{~Hz}, 1 \mathrm{H}), 4.12-4.08(\mathrm{~m}, 2 \mathrm{H}), 2.93-2.70$ (m, 3H), $2.15(\mathrm{~m}, 1 \mathrm{H}), 1.89-1.85(\mathrm{~m}, 2 \mathrm{H}), 1.74-1.72(\mathrm{~m}, 2 \mathrm{H}), 1.51-1.40(\mathrm{~m}, 12 \mathrm{H}) .{ }^{13} \mathbf{C}$ NMR $(100.6$ $\left.\mathrm{MHz}_{2} \mathrm{CDCl}_{3}\right) \delta 173.8,171.0,168.1,167.2,167.0,164.6,162.1,134.7,134.2,129.0,125.4,123.8$, $123.2,120.7,108.9,69.0,49.2,42.1,40.6,31.4,29.4,28.7,25.6,25.1,24.5,22.6$. HRMS-DART $(\mathrm{m} / \mathrm{z}):\left[\mathrm{M}+\mathrm{NH}_{4}\right]^{+}$calcd for $\mathrm{C}_{31} \mathrm{H}_{35} \mathrm{~N}_{4} \mathrm{O}_{9}{ }^{+}, 607.2399$; found, 607.2399. 


\section{Characterization Data for Redox Active Ester}

\section{1,3-Dioxoisoindolin-2-yl 3-Phenyloxetane-3-carboxylate (2l)}

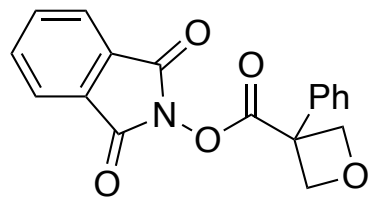

2)

The redox active ester $\mathbf{2 l}$ was purified by flash chromatography on silica gel (Biotage Selekt, 93:760:40, hexane/EtOAc). $1.0 \mathrm{mmol} \mathrm{scale;} 0.24 \mathrm{~g}, 0.74 \mathrm{mmol}, 74 \%$ isolated yield. White solid. M.p. 109-112 ${ }^{\circ} \mathrm{C}$. IR (neat) 695, 877, 967, 990, 1036, 1186, 1361, 1752, 1785, $1810 \mathrm{~cm}^{-1}$. ${ }^{\mathbf{1}} \mathbf{H}$ NMR $\left(400 \mathrm{MHz}, \mathrm{CDCl}_{3}\right) \delta 7.90-7.88(\mathrm{~m}, 2 \mathrm{H}), 7.81-8.79(\mathrm{~m}, 2 \mathrm{H}), 7.53-7.46(\mathrm{~m}, 4 \mathrm{H}), 7.40(\mathrm{~m}, 1 \mathrm{H}), 5.48$ $(\mathrm{d}, J=6.4 \mathrm{~Hz}, 2 \mathrm{H}), 5.10$ (d, $J=6.8 \mathrm{~Hz}, 2 \mathrm{H}) .{ }^{13} \mathbf{C ~ N M R}\left(100.6 \mathrm{MHz}, \mathrm{CDCl}_{3}\right) \delta 169.8,161.6,137.4$, 134.9, 129.1, 128.9, 128.4, 126.1, 124.1, 79.5, 51.8. HRMS-DART $(\mathrm{m} / \mathrm{z}):\left[\mathrm{M}+\mathrm{NH}_{4}\right]^{+}$calcd for $\mathrm{C}_{18} \mathrm{H}_{17} \mathrm{~N}_{2} \mathrm{O}_{5}, 341.1132$; found, 341.1120 .

\section{Preparation of Collidine $\cdot \mathrm{HBF}_{4}$}

To a $30 \mathrm{ml}$ round bottom flask with a stirring bar was added collidine $(1.32 \mathrm{ml}, 10.0 \mathrm{mmol})$ and $\mathrm{HBF}_{4}$ solution $\left(42 \mathrm{wt} \%, 2.09 \mathrm{~g}\right.$ ) at $0{ }^{\circ} \mathrm{C}$. After stirring at $0{ }^{\circ} \mathrm{C}$ for $20 \mathrm{~min}$, the water was removed under reduced pressure. The obtained white solid was washed with ether and dried under reduced pressure to afford collidine $\cdot \mathrm{HBF}_{4}(\mathbf{A 4}, 1.99 \mathrm{~g}, 9.5 \mathrm{mmol}, 95 \%$ isolated yield $)$.

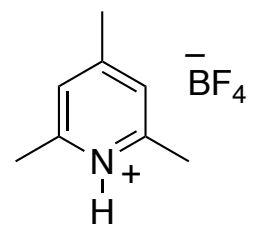

White solid. M.p. 236-237 ${ }^{\circ} \mathrm{C}$. IR (neat) 1057, 1398, 1634, 2342, 2359, 2986, 3088, 3181, 3134 , $3287 \mathrm{~cm}^{-1} .{ }^{1} \mathbf{H}$ NMR $\left(400 \mathrm{MHz}, \mathrm{DMSO}-d_{6}\right) \delta 7.58$ (s, 2H), 2.63 (s, 6H), 2.43 (s, 3H). ${ }^{13} \mathbf{C}$ NMR $\left(100.6 \mathrm{MHz}, \mathrm{DMSO}-d_{6}\right) \delta 158.5,151.9,125.0,21.3,19.0$. HRMS-DART $(\mathrm{m} / \mathrm{z}):\left[\mathrm{M}-\mathrm{BF}_{4}\right]^{+}$calcd for $\mathrm{C}_{8} \mathrm{H}_{12} \mathrm{~N}^{+}, 122.0964$; found, 122.0967 .

\section{Procedures for Decarboxyaltive N-Alkylation of Azoles}

Procedure A. In a glovebox, to an oven-dried vial with a stirring bar was added PTH1 (3.3 mg, 0.01 mmol), lithium tetrafluoroborate $(1.9 \mathrm{mg}, 0.02 \mathrm{mmol})$ and azole $1(0.2 \mathrm{mmol})$ and redox active ester $2(0.3 \mathrm{mmol})$. Then, ethyl acetate $(500 \mu \mathrm{L})$ was added to the reaction mixture. The reaction was stirred and irradiated with a $34 \mathrm{~W}$ blue LED $(0.5 \mathrm{~cm}$ away $)$ with a cooling fan to keep the temperature around $40{ }^{\circ} \mathrm{C}$ (Figure S1). After $24 \mathrm{~h}$, the reaction was quenched with a short plug of silica gel using ethyl acetate. After volatiles were removed under reduced pressure, purification by flash column chromatography on silica gel gave the $\mathrm{N}$-alkylated azole 3.

Procedure B. In a glovebox, to an oven-dried vial with a stirring bar was added PTH1 (3.3 mg, 0.01 mmol), 2,4,6-collidine $\cdot \mathrm{HBF}_{4} \mathbf{A 4}(83.6 \mathrm{mg}, 0.4 \mathrm{mmol})$ and azole $1(0.2 \mathrm{mmol})$ and redox active ester $2(0.4 \mathrm{mmol})$. Then, 1,2-dichloroethane $(500 \mu \mathrm{L})$ was added to the reaction mixture. The reaction was 
stirred and irradiated with a $34 \mathrm{~W}$ blue LED $(0.5 \mathrm{~cm}$ away) with a cooling fan to keep the temperature around $40^{\circ} \mathrm{C}$ (Figure S1). After $24 \mathrm{~h}$, the reaction was quenched with a short plug of silica gel using ethyl acetate. After volatiles were removed under reduced pressure, purification by flash column chromatography on silica gel gave the $\mathrm{N}$-alkylated azole $\mathbf{3}$.

Procedure C. In a glovebox, to an oven-dried vial with a stirring bar was added PTH1 (3.3 mg, 0.01 mmol), lithium tetrafluoroborate $(1.9 \mathrm{mg}, 0.02 \mathrm{mmol})$ and azole $1(0.2 \mathrm{mmol})$ and redox active ester $2(0.4 \mathrm{mmol})$. Then, 1,2-dichloroethane $(500 \mu \mathrm{L})$ was added to the reaction mixture. The reaction was stirred and irradiated with a $34 \mathrm{~W}$ blue LED $(0.5 \mathrm{~cm}$ away) with a cooling fan to keep the temperature around $40{ }^{\circ} \mathrm{C}$ (Figure S1). After $24 \mathrm{~h}$, the reaction was quenched with a short plug of silica gel using ethyl acetate. After volatiles were removed under reduced pressure, purification by flash column chromatography on silica gel gave the $\mathrm{N}$-alkylated azole $\mathbf{3}$.

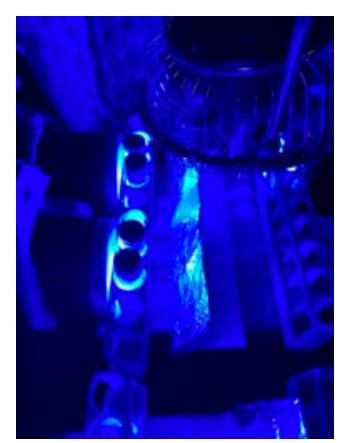

Figure S1. Light set up

1 mmol scale reaction of 3aa. In a glovebox, to an oven-dried vial with a stirring bar was added PTH1 (16.3 mg, $0.05 \mathrm{mmol}$ ), 2,4,6-collidine $\cdot \mathrm{HBF}_{4}$ (418 mg, $2.0 \mathrm{mmol}$ ) and azole 1a (197 mg, 1.0 mmol) and redox active ester $\mathbf{2 a}(495 \mathrm{mg}, 2.0 \mathrm{mmol})$. Then, 1,2-dichloroethane (2.5 $\mathrm{mL})$ was added to the reaction mixture. The reaction was stirred and irradiated with a $34 \mathrm{~W}$ blue LED ( $0.5 \mathrm{~cm}$ away) with a cooling fan to keep the temperature around $40{ }^{\circ} \mathrm{C}$ (Figure S2). After $24 \mathrm{~h}$, the reaction was quenched with a short plug of silica gel using ethyl acetate. After volatiles were removed under reduced pressure, purification by flash column chromatography (100:0-95:5, hexane/EtOAc) on silica gel gave the N-alkylated azole 3aa (135.4 mg, $0.53 \mathrm{mmol}, 53 \%$ isolated yield).

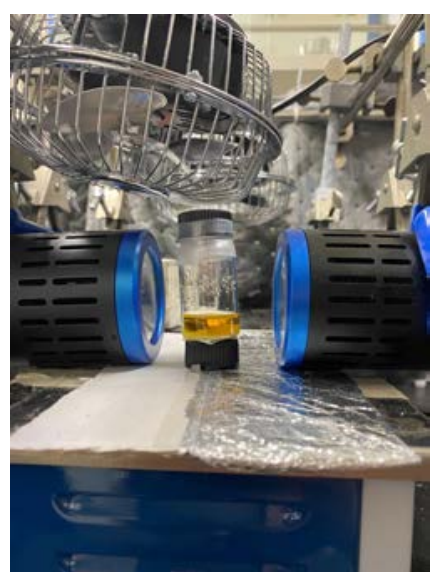

Figure S2. Light set up for scale-up reaction 


\section{Characterization Data for N-Alkylated Azoles}

\section{6-Bromo-1-(tert-butyl)-1H-indazole (3aa)}<smiles>CC(C)(C)n1ncc2ccc(Br)cc21</smiles>

The reaction was carried out according to procedure B and the reaction time was modified to $8 \mathrm{~h}$. The product 3aa was purified by flash chromatography on silica gel (100:0-95:5, hexane/EtOAc) (Table 1, entry 14; $29.9 \mathrm{mg}, 0.12 \mathrm{mmol}, 59 \%$ isolated yield). Yellow solid. M.p. $83-85{ }^{\circ} \mathrm{C}$. IR (neat) 593, 804, 920, 1036, 1112, 1205, 1371, 1457, 1621, $2977 \mathrm{~cm}^{-1} .{ }^{1} \mathbf{H}$ NMR (400 MHz, $\left.\mathrm{CDCl}_{3}\right) \delta 8.03$ (s, 1H), $7.91(\mathrm{~s}, 1 \mathrm{H}), 7.52(\mathrm{~d}, J=8.8 \mathrm{~Hz}, 1 \mathrm{H}), 7.13(\mathrm{dd}, J=8.8 \mathrm{~Hz}, 1.2 \mathrm{~Hz}, 1 \mathrm{H}), 1.73(\mathrm{~s}, 9 \mathrm{H}) .{ }^{13} \mathbf{C}$ NMR $\left(100.6 \mathrm{MHz}, \mathrm{CDCl}_{3}\right) \delta 149.0,125.0,121.6,119.9,119.8(\times 2 \mathrm{C}), 119.5,60.4,30.1$. HRMSDART $(\mathrm{m} / \mathrm{z})$ : $[\mathrm{M}+\mathrm{H}]^{+}$calcd for $\mathrm{C}_{11} \mathrm{H}_{14} \mathrm{BrN}_{2}, 253.0335$; found, 253.0340

\section{1-(tert-Butyl)-1H-indazole (3ba)}

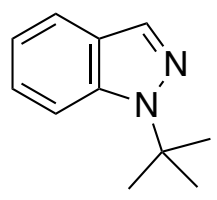

The reaction was carried out according to procedure B and the reaction time was modified to $8 \mathrm{~h}$. The product 3 ba was purified by flash chromatography on silica gel (100:0-95:5, hexane/EtOAc) (Table 2; $24.1 \mathrm{mg}, 0.14 \mathrm{mmol}, 69 \%$ isolated yield). Yellow oil. IR (neat) 756, 786, 1112, 1211, 1279, 1384, 1395, 1458, 1515, $1626 \mathrm{~cm}^{-1} .{ }^{1} \mathbf{H}$ NMR (400 MHz, $\left.\mathrm{CDCl}_{3}\right) \delta 8.05$ (s, 1H), $7.74(\mathrm{~d}, J=8.8 \mathrm{~Hz}$, 1H), $7.65(\mathrm{~d}, J=8.4 \mathrm{~Hz}, 1 \mathrm{H}), 7.27(\mathrm{~m}, 1 \mathrm{H}), 7.05(\mathrm{~m}, 1 \mathrm{H}), 1.75(\mathrm{~s}, 9 \mathrm{H}) .{ }^{13} \mathrm{C}$ NMR $(100.6 \mathrm{MHz}$, $\left.\mathrm{CDCl}_{3}\right) \delta 148.3,125.5,121.3,121.2,120.2,119.2,117.5,60.0,30.2$. HRMS-DART $(\mathrm{m} / \mathrm{z}):[\mathrm{M}+\mathrm{H}]^{+}$ calcd for $\mathrm{C}_{11} \mathrm{H}_{15} \mathrm{~N}_{2}$ 175.1230; found, 175.1229 .

\section{Methyl 1-(tert-Butyl)-1H-indazole-6-carboxylate (3ca)}

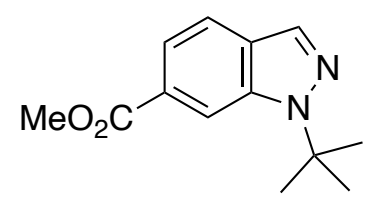

The reaction was carried out according to procedure B. The product $\mathbf{3 c a}$ was purified by flash chromatography on silica gel (100:0-85:15, hexane/EtOAc) (Table 2; $17.0 \mathrm{mg}, 0.07 \mathrm{mmol}, 37 \%$ isolated yield). Yellow solid. M.p. $96-99{ }^{\circ} \mathrm{C}$. IR (neat) 752, 1083, 1204, 1228, 1245, 1303, 1320, 1354, 1435, $1715 \mathrm{~cm}^{-1} .{ }^{1} \mathbf{H}$ NMR (400 MHz, $\left.\mathrm{CDCl}_{3}\right) \delta 8.56(\mathrm{~s}, 1 \mathrm{H}), 8.09$ (s, 1H), 7.69 (s, 2H), 3.94 $(\mathrm{s}, 3 \mathrm{H}), 1.76(\mathrm{~s}, 9 \mathrm{H}) .{ }^{13} \mathrm{C}$ NMR $\left(100.6 \mathrm{MHz}, \mathrm{CDCl}_{3}\right) \delta 167.7,147.5,127.4,123.4,121.5,120.9,120.2$, 119.5, 60.7, 52.1, 30.1. HRMS-DART $(\mathrm{m} / \mathrm{z})$ : $[\mathrm{M}+\mathrm{H}]^{+}$calcd for, $\mathrm{C}_{13} \mathrm{H}_{17} \mathrm{~N}_{2} \mathrm{O}_{2}, 233.1285$; found, 233.1293. 


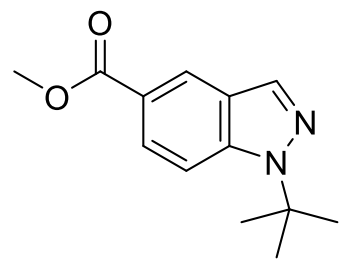

The reaction was carried out according to procedure B. The product 3da was purified by flash chromatography on silica gel (100:0-95:5, hexane/EtOAc) (Table 2; $38.4 \mathrm{mg}, 0.17 \mathrm{mmol}, 83 \%$ isolated yield). Yellow oil. IR (neat) 770, 1088, 1111, 1194, 1243, 1285, 1327,1375, 1435, 1627, $1711 \mathrm{~cm}^{-1} .{ }^{1} \mathbf{H}$ NMR $\left(400 \mathrm{MHz}, \mathrm{CDCl}_{3}\right) \delta 8.50(\mathrm{~s}, 1 \mathrm{H}), 8.19(\mathrm{~s}, 1 \mathrm{H}), 7.89(\mathrm{dd}, J=9.2,1.6 \mathrm{~Hz}, 1 \mathrm{H})$, $7.72(\mathrm{~d}, J=9.2 \mathrm{~Hz}, 1 \mathrm{H}), 3.93$ (s, 3H), $1.76(\mathrm{~s}, 9 \mathrm{H}) .{ }^{13} \mathrm{C}$ NMR $\left(100.6 \mathrm{MHz}, \mathrm{CDCl}_{3}\right) \delta 167.6,149.6$, 125.4, 125.0, 123.2, 121.8, 120.6, 117.2, 60.6, 51.9, 30.1. HRMS-DART $(\mathrm{m} / \mathrm{z}):[\mathrm{M}+\mathrm{H}]^{+}$calcd for $\mathrm{C}_{13} \mathrm{H}_{17} \mathrm{~N}_{2} \mathrm{O}_{2}, 233.1285$; found, 233.1279.

\section{tert-Butyl [1-(tert-Butyl)-1H-indazol-5-yl]carbamate (3ea)}

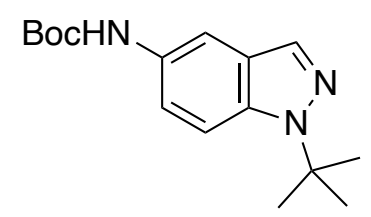

The reaction was carried out according to procedure B. The product 3ea was purified by flash chromatography on silica gel (100:0-90:10 hexane/EtOAc) (Table 2; $15.4 \mathrm{mg}, 0.06 \mathrm{mmol}, 28 \%$ isolated yield). White solid. IR (neat) 743, 809, 1049, 1162, 1241, 1326, 1366, 1520, 1718, $2976 \mathrm{~cm}^{-}$ ${ }^{1} .{ }^{1} \mathbf{H}$ NMR $\left(400 \mathrm{MHz} \mathrm{CDCl}_{3}\right) \delta 7.93(\mathrm{~s}, 1 \mathrm{H}), 7.86(\mathrm{~s}, 1 \mathrm{H}), 7.65(\mathrm{~d}, J=9.2 \mathrm{~Hz}, 1 \mathrm{H}), 7.01(\mathrm{dd}, J=$ $9.2 \mathrm{~Hz}, 2.0 \mathrm{~Hz}, 1 \mathrm{H}), 6.44$ (bs, $1 \mathrm{H}), 1.73$ (s, 9H), 1.53 (s, 9H). ${ }^{13} \mathbf{C ~ N M R}\left(100.6 \mathrm{MHz}, \mathrm{CDCl}_{3}\right) \delta 153.1$, $145.8,131.6,121.3,120.9,118.9,118.2,107.6,80.2,60.0,32.1,30.2,29.7,28.4$. HRMS-DART $(\mathrm{m} / \mathrm{z}):[\mathrm{M}+\mathrm{H}]^{+}$calcd for $\mathrm{C}_{16} \mathrm{H}_{24} \mathrm{~N}_{3} \mathrm{O}_{2}, 290.1863$; found, 290.1863 .

\section{1-(tert-Butyl)-4-Methyl-1H-indazole (3fa)}

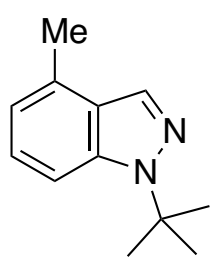

The reaction was carried out according to procedure B. The product $3 \mathrm{fa}$ was purified by flash chromatography on silica gel (100:0-95:5, hexane/EtOAc) (Table 2; $8.6 \mathrm{mg}, 0.05 \mathrm{mmol}, 23 \%$ isolated yield). Yellow soild. M.p. $25-27^{\circ} \mathrm{C}$. IR (neat) 768, 1119, 1211, 1372, 1384, 1397, 1408, 1458, 1517, $2976 \mathrm{~cm}^{-1} .{ }^{1} \mathbf{H}$ NMR $\left(400 \mathrm{MHz}, \mathrm{CDCl}_{3}\right) \delta 8.02(\mathrm{~s}, 1 \mathrm{H}), 7.57(\mathrm{~d}, J=8.8 \mathrm{~Hz}, 1 \mathrm{H}), 7.17(\mathrm{~m}, 1 \mathrm{H}), 6.81$ $(\mathrm{d}, J=6.8 \mathrm{~Hz}, 1 \mathrm{H}), 2.53(\mathrm{~s}, 3 \mathrm{H}), 1.75(\mathrm{~s}, 9 \mathrm{H}) .{ }^{13} \mathbf{C ~ N M R}\left(100.6 \mathrm{MHz}, \mathrm{CDCl}_{3}\right) \delta 148.3,130.2,125.9$, $122.5, \quad 120.4, \quad 118.2, \quad 114.8, \quad 59.9, \quad 30.2, \quad 19.2$. HRMS-DART $(m / z):[\mathrm{M}+\mathrm{H}]^{+}$calcd for $\mathrm{C}_{12} \mathrm{H}_{17} \mathrm{~N}_{2}, 189.1386$; found, 189.1389 . 
<smiles>CC(C)(C)n1nnc2ccccc21</smiles>

$3 g a$<smiles>CC(C)(C)n1nc2ccccc2n1</smiles>

3ga'

The reaction was carried out according to procedure B. The product was purified by flash chromatography on silica gel (100:0-95:5, hexane/EtOAc) (Table 2; 3ga; 9.4 mg, 0.05 mmol, 27\% isolated yield, 3ga'; $11.0 \mathrm{mg}, 0.06 \mathrm{mmol}, 31 \%$ isolated yield). The spectrum data of product 3ga or 3ga' was consistent with the literature, respectively. ${ }^{6}$

\section{Ethyl 1-(tert-Butyl)-1H-pyrazole-4-carboxylate (3ha)}

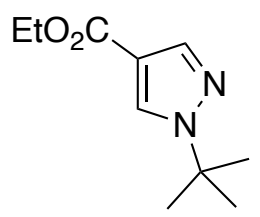

The reaction was carried out according to procedure B with addition of acetonitrile $(10 \mu \mathrm{L})$. The product 3ha was purified by flash chromatography on silica gel (100:0-90:10, hexane/EtOAc). (Table 2; $19.7 \mathrm{mg}, 0.10 \mathrm{mmol}, 50 \%$ isolated yield). The spectrum data of product $\mathbf{3 h a}$ was consistent with the literature. ${ }^{7}$

\section{6-Bromo-1-(2-phenylpropan-2-yl)-1H-indazole (3ab)}<smiles>CC(C)(c1ccccc1)n1ncc2ccc(Br)cc21</smiles>

The reaction was carried out according to procedure A. The product 3ab was purified by flash chromatography on silica gel (100:0-95:5, hexane/EtOAc) (Table 2; $61.4 \mathrm{mg}, 0.19 \mathrm{mmol}, 97 \%$ isolated yield). Yellow oil. IR (neat) 698, 805, 920, 1033, 1249, 1386, 1448, 1621, $2984 \mathrm{~cm}^{-1} .{ }^{1} \mathbf{H}$ NMR (400 MHz, $\left.\mathrm{CDCl}_{3}\right) \delta 7.92(\mathrm{~d}, J=6.0 \mathrm{~Hz}, 2 \mathrm{H}), 7.50(\mathrm{~d}, J=8.8 \mathrm{~Hz}, 1 \mathrm{H}), 7.33-7.26(\mathrm{~m}, 3 \mathrm{H})$, $7.14(\mathrm{~d}, J=8.8 \mathrm{~Hz}, 1 \mathrm{H}), 7.07(\mathrm{~d}, J=7.6 \mathrm{~Hz}, 2 \mathrm{H}), 2.11(\mathrm{~s}, 6 \mathrm{H}) .{ }^{13} \mathbf{C} \mathbf{N M R}\left(100.6 \mathrm{MHz}, \mathrm{CDCl}_{3}\right)$ $\delta 148.9,145.6,128.6,127.6,125.3,125.1,122.1,121.7,120.3,120.0,119.7,65.6,30.0$. HRMSDART $(m / z):[\mathrm{M}+\mathrm{H}]^{+}$calcd for $\mathrm{C}_{16} \mathrm{H}_{16} \mathrm{BrN}_{2}, 315.0491$; found, 315.0484 .

\section{2-[1-(2-Phenylpropan-2-yl)-1H-indazol-5-yl]isoindoline-1,3-dione (3ib)}

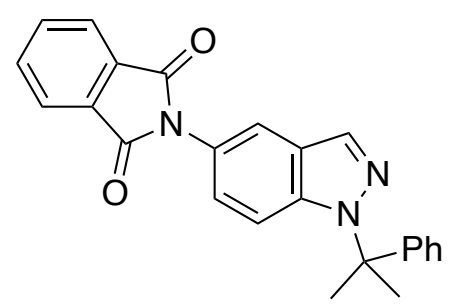


The reaction was carried out according to procedure A. The product $3 \mathbf{i b}$ was purified by flash chromatography on silica gel (100:0-70:30, hexane/EtOAc) (Scheme 3; $38.0 \mathrm{mg}, 0.10 \mathrm{mmol}, 50 \%$ isolated yield). Yellow solid. M.p. $141-146^{\circ} \mathrm{C}$. IR (neat) $638,718,805,1083,1110,1179,1368$, 1523, 1716, $2984 \mathrm{~cm}^{-1} .{ }^{1} \mathbf{H}$ NMR (400 MHz, $\left.\mathrm{CDCl}_{3}\right) \delta 8.03(\mathrm{~s}, 1 \mathrm{H}), 7.97(\mathrm{dd}, J=5.2 \mathrm{~Hz}, 3.2 \mathrm{~Hz}$, 2H), $7.87(\mathrm{~d}, J=8.8 \mathrm{~Hz}, 1 \mathrm{H}), 7.80(\mathrm{dd}, J=5.6 \mathrm{~Hz}, 3.2 \mathrm{~Hz}, 2 \mathrm{H}), 7.69$ (s, 1H), 7.34-7.27 (m, 4H), 7.09 $(\mathrm{d}, J=7.2 \mathrm{~Hz}, 2 \mathrm{H}), 2.14$ (s, 6H). ${ }^{13} \mathbf{C}$ NMR (100.6 MHz, $\left.\mathrm{CDCl}_{3}\right) \delta 167.7,147.3,145.7,134.3,131.9$, 128.6, 127.6, 125.2, 125.1, 124.9, 123.7, 122.6, $121.1119 .1,119.0,65.7,30.1$. HRMS-DART $(\mathrm{m} / \mathrm{z})$ : $[\mathrm{M}+\mathrm{H}]^{+}$calcd for $\mathrm{C}_{24} \mathrm{H}_{20} \mathrm{~N}_{3} \mathrm{O}_{2}, 382.1550$; found, 382.1547 .

\section{Methyl 1-(2-Phenylpropan-2-yl)-1H-indazole-5-carboxylate (3db)}

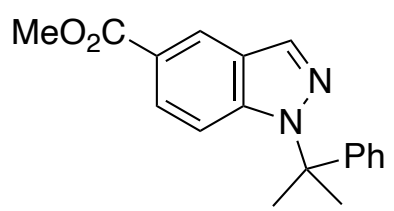

The reaction was carried out according to procedure B. The product $\mathbf{3 d b}$ was purified by flash chromatography on silica gel (100:0-95:5, hexane/EtOAc) (Table 2; $47.5 \mathrm{mg}, 0.16 \mathrm{mmol}, 81 \%$ isolated yield). Yellow oil. IR (neat) 698, 734, 1086, 1165, 1375, 1434, 1496, 1627, 1709, $2984 \mathrm{~cm}^{-}$ ${ }^{1} .{ }^{1} \mathbf{H}$ NMR $\left(400 \mathrm{MHz} \mathrm{CDCl}_{3}\right) \delta 8.48(\mathrm{~s}, 1 \mathrm{H}), 8.08(\mathrm{~s}, 1 \mathrm{H}), 7.90(\mathrm{~d}, J=9.2 \mathrm{~Hz}, 1 \mathrm{H}), 7.74(\mathrm{~d}, J=9.2$ $\mathrm{Hz}, 1 \mathrm{H}), 7.34-7.28(\mathrm{~m}, 3 \mathrm{H}), 7.10(\mathrm{~d}, J=7.2 \mathrm{~Hz}, 2 \mathrm{H}), 3.93(\mathrm{~s}, 3 \mathrm{H}), 2.14(\mathrm{~s}, 6 \mathrm{H}) .{ }^{13} \mathbf{C}$ NMR $(100.6$ $\left.\mathrm{MHz}, \mathrm{CDCl}_{3}\right) \delta 167.6,149.5,145.4,128.7,127.7,125.6,125.1(\times 2 \mathrm{C}), 124.0,123.7,120.9,117.6$, 65.8, 52.0, 30.0. HRMS-DART $(\mathrm{m} / \mathrm{z}):[\mathrm{M}+\mathrm{H}]^{+}$calcd for $\mathrm{C}_{18} \mathrm{H}_{19} \mathrm{~N}_{2} \mathrm{O}_{2}, 295.1441$; found, 295.1438 .

\section{Ethyl 1-(tert-Butyl)-3-(trifluoromethyl)-1H-pyrazole-4-carboxylate (3jb)}

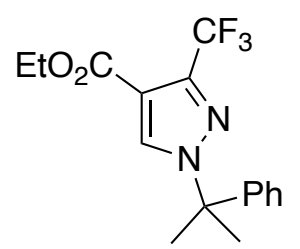

The reaction was carried out according to procedure $\mathrm{A}$ and dichloromethane $(500 \mu \mathrm{L})$ was instead of ethyl acetate $(500 \mu \mathrm{L})$. The product $\mathbf{3 j b}$ was purified by flash chromatography on silica gel (100:095:5, hexane/EtOAc) (Table 2; $23.5 \mathrm{mg}, 0.07 \mathrm{mmol}, 36 \%$ isolated yield). Yellow oil. IR (neat) 699, $775,1051,1141,1183,1211,1183,1304,1497,1540,1732 \mathrm{~cm}^{-1} .{ }^{1} \mathbf{H}$ NMR $\left(400 \mathrm{MHz}, \mathrm{CDCl}_{3}\right) \delta$ $7.92(\mathrm{~s}, 1 \mathrm{H}), 7.37-7.29(\mathrm{~m}, 3 \mathrm{H}), 7.13$ (d, $J=7.2 \mathrm{~Hz}, 2 \mathrm{H}), 4.30$ (q, $J=7.2 \mathrm{~Hz}, 2 \mathrm{H}), 1.99$ (s, 6H), 1.33 $(\mathrm{t}, J=6.8 \mathrm{~Hz}, 3 \mathrm{H}) .{ }^{13} \mathbf{C} \mathbf{N M R}\left(100.6 \mathrm{MHz}, \mathrm{CDCl}_{3}\right) \delta 161.2,144.2,134.2,128.8,128.0,126.9\left(\mathrm{q}, J_{\mathrm{C}-}\right.$ $\mathrm{F}=251.6 \mathrm{~Hz}), 120.5\left(\mathrm{q}, J_{\mathrm{C}-\mathrm{F}}=269.6 \mathrm{~Hz}\right), 65.5,60.8,29.4,14.0$. HRMS-DART $(\mathrm{m} / \mathrm{z}):[\mathrm{M}+\mathrm{H}]^{+}$calcd for $\mathrm{C}_{11} \mathrm{H}_{16} \mathrm{~F}_{3} \mathrm{~N}_{2} \mathrm{O}_{2}, 327.1315$; found, 327.1314 . 


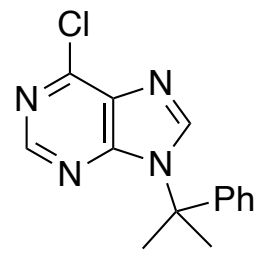

The reaction was carried out according to procedure B. The product $\mathbf{3 k b}$ was purified by flash chromatography on silica gel (100:0-85:15, hexane/EtOAc). (Table 2; $30.0 \mathrm{mg}, 0.11 \mathrm{mmol}, 55 \%$ isolated yield). The spectrum data of product $\mathbf{3 k b}$ was consistent with the literature. ${ }^{8}$

\section{1-(2-Phenylpropan-2-yl)-1H-pyrrolo[3,2-c]pyridine (3lb)}

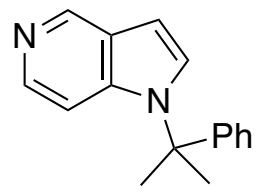

The reaction was carried out according to procedure $\mathrm{C}$. The product 3lb was purified by flash chromatography on silica gel (Biotage Selekt, 93:7-40:60, hexane/EtOAc) (Table 2; $15.9 \mathrm{mg}, 0.07$ mmol, 34\% isolated yield). Yellow oil. IR (neat) 702, 744, 891, 1078, 1128, 1183, 1239, 1312, 1453, $1708 \mathrm{~cm}^{-1} .{ }^{1} \mathbf{H}$ NMR $\left(400 \mathrm{MHz}, \mathrm{CDCl}_{3}\right) \delta 8.81(\mathrm{~s}, 1 \mathrm{H}), 8.53(\mathrm{~s}, 1 \mathrm{H}), 8.41(\mathrm{~d}, J=5.6 \mathrm{~Hz}, 1 \mathrm{H}), 7.40-$ $7.37(\mathrm{~m}, 2 \mathrm{H}), 7.32-7.28(\mathrm{~m}, 3 \mathrm{H}), 6.87(\mathrm{~d}, J=4.0 \mathrm{~Hz}, 1 \mathrm{H}), 6.37(\mathrm{~d}, J=3.6 \mathrm{~Hz}, 1 \mathrm{H}), 1.76(\mathrm{~s}, 6 \mathrm{H}) .{ }^{13} \mathrm{C}$ NMR (100.6 MHz, $\left.\mathrm{CDCl}_{3}\right) \delta 175.8,145.1,144.5,143.3,140.9,129.5,127.4,127.2,125.1,111.8$, 106.4, 49.8, 28.2. HRMS-DART (m/z): $\left[\mathrm{M}+\mathrm{NH}_{4}\right]^{+}$calcd for $\mathrm{C}_{16} \mathrm{H}_{17} \mathrm{~N}_{2}, 237.1386$; found, 237.1381 .

1-(2-Phenylpropan-2-yl)-1H-benzo[d][1,2,3]triazole (3gb) and 2-(2-Phenylpropan-2-yl)-2Hbenzo[d] $[1,2,3]$ triazole $(3 \mathrm{gb}$ ')<smiles>CC(C)(c1ccccc1)n1nnc2ccccc21</smiles>

$3 g b$

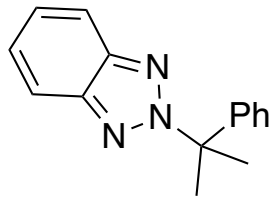

$3 g b^{\prime}$

The reaction was carried out according to procedure A. The product was purified by flash chromatography on silica gel (100:0-90:10, hexane/EtOAc) (Table 2; 3gb; $22.3 \mathrm{mg}, 0.09$ mmol, 47\% isolated yield, 3gb'; $14.2 \mathrm{mg}, 0.06 \mathrm{mmol}, 32 \%$ isolated yield). The spectrum data of product $\mathbf{3} \mathbf{g b}^{7}$ or $\mathbf{3 g b}^{7}{ }^{7}$ was consistent with the literature, respectively.

\section{Methyl 1-(tert-Pentyl)-1H-indazole-5-carboxylate (3dc)}<smiles>CCC(C)(C)n1ncc2cc(C(O)OC)ccc21</smiles> 
The reaction was carried out according to procedure B. The product $\mathbf{3 d c}$ was purified by flash chromatography on silica gel (100:0-95:5, hexane/EtOAc) (Table 2; $34.1 \mathrm{mg}, 0.14 \mathrm{mmol}$, 69\% isolated yield). Yellow oil. IR (neat) 769, 1087, 1228, 1276, 1377, 1434, 1626, 1709, $2972 \mathrm{~cm}^{-1} .{ }^{1} \mathbf{H}$ NMR $\left(400 \mathrm{MHz}, \mathrm{CDCl}_{3}\right) \delta 8.51(\mathrm{~s}, 1 \mathrm{H}), 8.15(\mathrm{~s}, 1 \mathrm{H}), 7.88(\mathrm{dd}, J=8.8 \mathrm{~Hz}, 1.6 \mathrm{~Hz}, 1 \mathrm{H}), 7.72(\mathrm{~d}, J=$ $8.8 \mathrm{~Hz}, 1 \mathrm{H}), 3.93(\mathrm{~s}, 3 \mathrm{H}), 2.08(\mathrm{q}, J=7.6 \mathrm{~Hz}, 2 \mathrm{H}), 1.73(\mathrm{~s}, 6 \mathrm{H}), 0.68(\mathrm{t}, J=7.6 \mathrm{~Hz}, 3 \mathrm{H}) .{ }^{13} \mathbf{C} \mathbf{N M R}$ $\left(100.6 \mathrm{MHz} \mathrm{CDCl}_{3}\right) \delta 167.6,149.6,125.4,125.0,123.2,122.7,120.4,117.2,63.5,51.9,35.5,27.4$, 8.2. HRMS-DART $(\mathrm{m} / \mathrm{z})$ : $[\mathrm{M}+\mathrm{H}]^{+}$calcd for $\mathrm{C}_{14} \mathrm{H}_{19} \mathrm{~N}_{2} \mathrm{O}_{2}, 247.1441$; found,247.1441.

\section{1-(2-Methyl-1-phenylpropan-2-yl)-1H-indazole (3bd)}

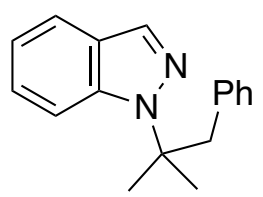

The reaction was carried out according to procedure B. The product $\mathbf{3 b d}$ was purified by flash chromatography on silica gel (100:0-95:5, hexane/EtOAc) (Table 2; $11.8 \mathrm{mg}, 0.05 \mathrm{mmol}, 24 \%$ isolated yield). Yellow solid. M.p. $83-85^{\circ} \mathrm{C}$. IR (neat) 703, 744, 756, 781, 1105, 1137, 1170, 1370, 1387, $1455 \mathrm{~cm}^{-1} .{ }^{1} \mathbf{H}$ NMR $\left(400 \mathrm{MHz}, \mathrm{CDCl}_{3}\right) \delta 7.80(\mathrm{~d}, J=8.8 \mathrm{~Hz}, 1 \mathrm{H}), 7.71(\mathrm{~s}, 1 \mathrm{H}), 7.59(\mathrm{~d}, J=$ $8.4 \mathrm{~Hz}, 1 \mathrm{H}), 7.31(\mathrm{t}, J=8.4 \mathrm{~Hz}, 1 \mathrm{H}), 7.16-7.04(\mathrm{~m}, 4 \mathrm{H}), 6.61(\mathrm{~d}, J=7.6 \mathrm{~Hz}, 2 \mathrm{H}), 3.32(\mathrm{~s}, 2 \mathrm{H}), 1.73(\mathrm{~s}$, $6 \mathrm{H}) .{ }^{13} \mathrm{C}$ NMR $\left(100.6 \mathrm{MHz}, \mathrm{CDCl}_{3}\right) \delta 148.5,136.6,129.9,127.9,126.6,125.7,121.1121 .0(\times 2 \mathrm{C})$, 120.4, 117.4, 62.9, 49.0, 27.7. HRMS-DART $(\mathrm{m} / \mathrm{z})$ : $[\mathrm{M}+\mathrm{H}]^{+}$calcd for, $\mathrm{C}_{17} \mathrm{H}_{19} \mathrm{~N}_{2}, 251.1543$; found, 251.1507 .

\section{1-(1-Methylcyclobutyl)-1H-indazole (3be)}

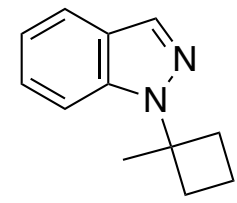

The reaction was carried out according to procedure B. The product 3 be was purified by flash chromatography on silica gel (100:0-97:3, hexane/EtOAc) (Table 2; $20.2 \mathrm{mg}, 0.11 \mathrm{mmol}, 54 \%$ isolated yield). Yellow oil. IR (neat) 755, 782, 1104, 1190, 1284, 1350, 1384, 1514, 1627, $2971 \mathrm{~cm}^{-}$ 1. ${ }^{1} \mathbf{H}$ NMR $\left(400 \mathrm{MHz}, \mathrm{CDCl}_{3}\right) \delta 7.96(\mathrm{~s}, 1 \mathrm{H}), 7.75(\mathrm{dd}, J=8.8 \mathrm{~Hz}, 0.8 \mathrm{~Hz}, 1 \mathrm{H}), 7.65(\mathrm{~d}, J=8.4$ $\mathrm{Hz}, 1 \mathrm{H}), 7.27$ (m, 1H), 7.07 (m, 1H), 2.92-2.84 (m, 2H), 2.41-2.35 (m, 2H), 2.07-1.97 (m, 2H), 1.83 (s, 1H). ${ }^{13} \mathbf{C}$ NMR $\left(100.6 \mathrm{MHz}, \mathrm{CDCl}_{3}\right) \delta$ 148.5, 125.6, 121.5, 121.4, 120.1, 119.3, 117.5, 62.3, 34.7, 27.4, 14.1. HRMS-DART $(\mathrm{m} / \mathrm{z})$ : $[\mathrm{M}+\mathrm{H}]^{+}$calcd for $\mathrm{C}_{12} \mathrm{H}_{15} \mathrm{~N}_{2}, 187.1230$; found, 187.1228 .

\section{1-(1-Methylcyclopentyl)-1H-indazole (3bf)}

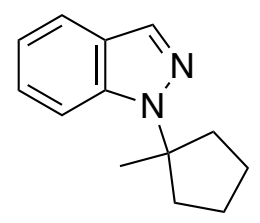


The reaction was carried out according to procedure $\mathrm{B}$. The product $\mathbf{3 b f}$ was purified by flash chromatography on silica gel (100:0-97:3, hexane/EtOAc) (Table 2; $23.6 \mathrm{mg}, 0.12 \mathrm{mmol}, 59 \%$ isolated yield). Yellow oil. IR (neat) 668, 754, 780, 974, 1112, 1212, 1384, 1513, 1627, $2966 \mathrm{~cm}^{-1}$. ${ }^{1} \mathbf{H}$ NMR $\left(400 \mathrm{MHz}, \mathrm{CDCl}_{3}\right) \delta 8.04(\mathrm{~s}, 1 \mathrm{H}), 7.74(\mathrm{~d}, J=8.4 \mathrm{~Hz}, 1 \mathrm{H}), 7.65(\mathrm{~d}, J=8.4 \mathrm{~Hz}, 1 \mathrm{H}), 7.26$ $(\mathrm{m}, 1 \mathrm{H}), 7.05(\mathrm{t}, J=7.6 \mathrm{~Hz}, 1 \mathrm{H}), 2.59-2.52(\mathrm{~m}, 2 \mathrm{H}), 2.10-2.03(\mathrm{~m}, 2 \mathrm{H}), 1.85-1.82(\mathrm{~m}, 4 \mathrm{H}), 1.71(\mathrm{~s}$, $3 \mathrm{H}) .{ }^{13} \mathrm{C}$ NMR $\left(100.6 \mathrm{MHz}, \mathrm{CDCl}_{3}\right) \delta 148.3,125.5,121.4,121.2,120.1,120.0,117.5,70.6,39.8$, 28.5, 23.5. HRMS-DART $(\mathrm{m} / \mathrm{z})$ : $[\mathrm{M}+\mathrm{H}]^{+}$calcd for $\mathrm{C}_{13} \mathrm{H}_{17} \mathrm{~N}_{2}, 201.1386$; found, 201.1381.

\section{1-(1-Methylcyclohexyl)-1H-indazole (3bg)}

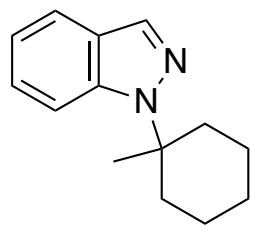

The reaction was carried out according to procedure B. The product $\mathbf{3 b g}$ was purified by flash chromatography on silica gel (100:0-97:3, hexane/EtOAc) (Table 2; $16.8 \mathrm{mg}, 0.08 \mathrm{mmol}, 39 \%$ isolated yield). Colorless oil. IR (neat) 754, 1108, 1142, 1173, 1292, 1386, 1449, 1627, 2862, 2933 $\mathrm{cm}^{-1} .{ }^{1} \mathbf{H}$ NMR $\left(400 \mathrm{MHz}, \mathrm{CDCl}_{3}\right) \delta 8.10(\mathrm{~s}, 1 \mathrm{H}), 7.75(\mathrm{~d}, J=8.0 \mathrm{~Hz}, 1 \mathrm{H}), 7.66(\mathrm{~d}, J=8.0 \mathrm{~Hz}, 1 \mathrm{H})$, $7.26(\mathrm{~m}, 1 \mathrm{H}), 7.06(\mathrm{t}, J=7.6 \mathrm{~Hz}, 1 \mathrm{H}), 2.53-2.47(\mathrm{~m}, 2 \mathrm{H}), 1.97-1.91(\mathrm{~m}, 2 \mathrm{H}), 1.69-1.60(\mathrm{~m}, 3 \mathrm{H})$, $1.58(\mathrm{~s}, 3 \mathrm{H}), 1.56-1.43(\mathrm{~m}, 3 \mathrm{H}) .{ }^{13} \mathbf{C} \mathbf{N M R}\left(100.6 \mathrm{MHz}, \mathrm{CDCl}_{3}\right) \delta 148.1,125.4,121.3,121.2,120.2$, 119.6, 117.6, 62.6, 37.5, 29.1, 25.3, 22.3. HRMS-DART $(\mathrm{m} / \mathrm{z}):[\mathrm{M}+\mathrm{H}]^{+}$calcd for $\mathrm{C}_{14} \mathrm{H}_{19} \mathrm{~N}_{2}$, 215.1543; found, 215.1547.

\section{1-[5-(2,5-Dimethylphenoxy)-2-methylpentan-2-yl]-1H-indazole (3bn)}

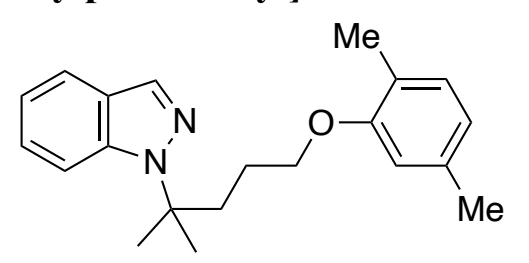

The reaction was carried out according to procedure B. The product $\mathbf{3 b n}$ was purified by flash chromatography on silica gel (100:0-97:3, hexane/EtOAc) (Table 2; $33.8 \mathrm{mg}, 0.10 \mathrm{mmol}, 52 \%$ isolated yield). Pale yellow solid. M.p. $89-90{ }^{\circ} \mathrm{C}$. IR (neat) 755, 804, 1048, 1111, 1129, 1157, 1264, 1284, 1389, $1509 \mathrm{~cm}^{-1} .{ }^{1} \mathbf{H}$ NMR $\left(400 \mathrm{MHz}, \mathrm{CDCl}_{3}\right) \delta 8.07(\mathrm{~s}, 1 \mathrm{H}), 7.74(\mathrm{dd}, J=8.8 \mathrm{~Hz}, 0.8 \mathrm{~Hz}$, 1H), $7.66(\mathrm{~d}, J=8.4 \mathrm{~Hz}, 1 \mathrm{H}), 7.27(\mathrm{~m}, 1 \mathrm{H}), 7.06(\mathrm{~m}, 1 \mathrm{H}), 6.98(\mathrm{~d}, J=7.6 \mathrm{~Hz}, 1 \mathrm{H}), 6.63(\mathrm{~d}, J=7.6$ $\mathrm{Hz}, 1 \mathrm{H}), 6.50(\mathrm{~s}, 1 \mathrm{H}), 3.82(\mathrm{t}, J=8.4 \mathrm{~Hz}, 2 \mathrm{H}), 2.26-2.22(\mathrm{~m}, 5 \mathrm{H}), 2.15(\mathrm{~s}, 3 \mathrm{H}), 1.78(\mathrm{~s}, 6 \mathrm{H}), 1.55-$ $1.50(\mathrm{~m}, 2 \mathrm{H}) .{ }^{13} \mathbf{C ~ N M R}\left(100.6 \mathrm{MHz}, \mathrm{CDCl}_{3}\right) \delta$ 156.8, 148.4, 136.4, 130.2, 125.6, 123.4, 121.3, $121.2,120.7,120.2,120.1,117.5,111.8,67.3,62.3,39.4,28.1,24.1,21.3,15.8$. HRMS-DART $(\mathrm{m} / \mathrm{z}):[\mathrm{M}+\mathrm{H}]^{+}$calcd for $\mathrm{C}_{21} \mathrm{H}_{27} \mathrm{~N}_{2} \mathrm{O}, 323.21179$; found, 322.21228 . 


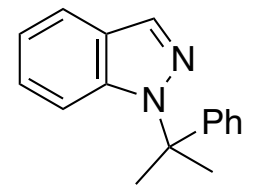

The reaction was carried out according to procedure B. The product $\mathbf{3 b b}$ was purified by flash chromatography on silica gel (100:0-97:3, hexane/EtOAc) (Table 2; $38.8 \mathrm{mg}, 0.16 \mathrm{mmol}, 82 \%$ isolated yield). Yellow oil. IR (neat) 699, 756, 783, 1114, 1181, 1350, 1368, 1388, 1448, $1497 \mathrm{~cm}^{-1}$. ${ }^{1} \mathbf{H}$ NMR $\left(400 \mathrm{MHz}, \mathrm{CDCl}_{3}\right) \delta 7.95(\mathrm{~s}, 1 \mathrm{H}), 7.76(\mathrm{dd}, J=8.8 \mathrm{~Hz}, 0.8 \mathrm{~Hz}, 1 \mathrm{H}), 7.64(\mathrm{dt}, J=8.4 \mathrm{~Hz}$, $0.8 \mathrm{~Hz}, 1 \mathrm{H}), 7.32-7.24(\mathrm{~m}, 4 \mathrm{H}), 7.09-7.05(\mathrm{~m}, 3 \mathrm{H}), 2.13(\mathrm{~s}, 6 \mathrm{H}) .{ }^{13} \mathbf{C} \mathbf{N M R}\left(100.6 \mathrm{MHz}, \mathrm{CDCl}_{3}\right)$ $\delta 148.2,146.0,128.5,127.4,125.7,125.1,121.5,121.4,120.2(\times 2 \mathrm{C}), 117.8,65.2,30.1$. HRMSDART $(\mathrm{m} / \mathrm{z})$ : $[\mathrm{M}+\mathrm{H}]^{+}$calcd for $\mathrm{C}_{16} \mathrm{H}_{17} \mathrm{~N}_{2}, 237.1386$; found, 237.1384 .

\section{Methyl 1-(2-Phenylbutan-2-yl)-1H-indazole-5-carboxylate (3di)}

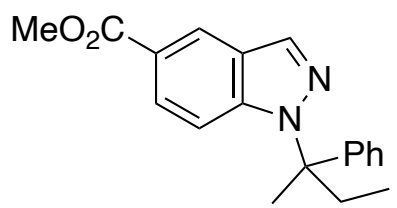

The reaction was carried out according to procedure B. The product 3di was purified by flash chromatography on silica gel (100:0-95:5, hexane/EtOAc) (Table 2; $44.7 \mathrm{mg}, 0.14 \mathrm{mmol}, 72 \%$ isolated yield). Yellow oil. IR (neat) 674, 769, 965, 1087, 1184, 1276, 1434, 1626, 1709, $2972 \mathrm{~cm}^{-1}$. ${ }^{1} \mathbf{H}$ NMR $\left(400 \mathrm{MHz}, \mathrm{CDCl}_{3}\right) \delta 8.49$ (s, 1H), 8.09 (s, 1H), 7.89 (dd, $\left.J=9.2 \mathrm{~Hz}, 1.6 \mathrm{~Hz}, 1 \mathrm{H}\right), 7.74$ (d, $J=9.2 \mathrm{~Hz}, 1 \mathrm{H}), 7.32-7.24(\mathrm{~m}, 3 \mathrm{H}), 7.05-7.07(\mathrm{~m}, 2 \mathrm{H}), 3.92(\mathrm{~s}, 3 \mathrm{H}), 2.73(\mathrm{~m}, 1 \mathrm{H}), 2.45(\mathrm{~m}, 1 \mathrm{H})$, $2.05(\mathrm{~s}, 3 \mathrm{H}), 0.80(\mathrm{t}, J=7.2 \mathrm{~Hz}, 3 \mathrm{H}) .{ }^{13} \mathbf{C} \mathbf{N M R}\left(100.6 \mathrm{MHz}, \mathrm{CDCl}_{3}\right) \delta 167.5,149.4,145.0,128.5$, $127.5,125.5,125.4,125.1,124.6,123.5,120.6,117.6,69.0,51.9,33.9,26.5,8.4$. HRMS-DART $(\mathrm{m} / \mathrm{z}):[\mathrm{M}+\mathrm{H}]^{+}$calcd for $\mathrm{C}_{19} \mathrm{H}_{21} \mathrm{~N}_{2} \mathrm{O}_{2}, 309.1598$; found, 309.1594 .

\section{Methyl 1-(1-Phenylcyclobutyl)-1H-indazole-5-carboxylate (3dj)}

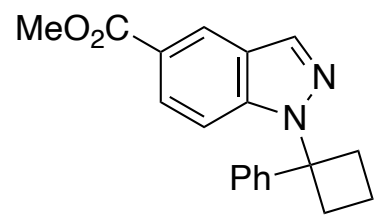

The reaction was carried out according to procedure B. The product 3dj was purified by flash chromatography on silica gel (100:0-95:5, hexane/EtOAc) (Table 2; $44.0 \mathrm{mg}, 0.14 \mathrm{mmol}, 72 \%$ isolated yield). Yellow solid. M.p. $74-76{ }^{\circ} \mathrm{C}$. IR (neat) 771, 1089, 1153, 1243, 1277, 1376, 1434, 1627, 1712, $2945 \mathrm{~cm}^{-1} .{ }^{1} \mathbf{H}$ NMR $\left(400 \mathrm{MHz} \mathrm{CDCl}_{3}\right) \delta 8.43(\mathrm{~s}, 1 \mathrm{H}), 7.90-7.87(\mathrm{~m}, 2 \mathrm{H}), 7.75(\mathrm{~d}, J=$ 9.2 Hz, 1H), 7.39-7.32 (m, 5H), $3.92(\mathrm{~s}, 3 \mathrm{H}), 3.34-3.27(\mathrm{~m}, 2 \mathrm{H}), 3.04-2.97(\mathrm{~m}, 2 \mathrm{H}), 2.17(\mathrm{~m}, 1 \mathrm{H})$, $2.05(\mathrm{~m}, 1 \mathrm{H}) .{ }^{13} \mathbf{C ~ N M R}\left(100.6 \mathrm{MHz}, \mathrm{CDCl}_{3}\right) \delta 167.5,149.7,142.7,128.7,128.0,126.0,125.6,125.0$, 123.9, 123.6, 121.0, 117.5, 68.6, 52.0, 35.0, 14.9. HRMS-DART $(\mathrm{m} / z):[\mathrm{M}+\mathrm{H}]^{+}$calcd for $\mathrm{C}_{19} \mathrm{H}_{19} \mathrm{~N}_{2} \mathrm{O}_{2}, 307.1491$; found, 307.1483. 


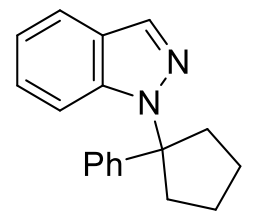

The reaction was carried out according to procedure B. The product $\mathbf{3 b k}$ was purified by flash chromatography on silica gel (100:0-97:3, hexane/EtOAc) (Table 2; $21.8 \mathrm{mg}, 0.08 \mathrm{mmol}, 42 \%$ isolated yield). Yellow solid. M.p. 90-92 ${ }^{\circ} \mathrm{C}$. IR (neat) 698, 754, 780, 1112, 1331, 1388, 1448, 1496, 1513, $2961 \mathrm{~cm}^{-1} .{ }^{1} \mathbf{H}$ NMR $\left(400 \mathrm{MHz}, \mathrm{CDCl}_{3}\right) \delta 8.02(\mathrm{~s}, 1 \mathrm{H}), 7.75(\mathrm{~d}, J=8.8 \mathrm{~Hz}, 1 \mathrm{H}), 7.62(\mathrm{~d}, J=$ $8.4 \mathrm{~Hz}, 1 \mathrm{H}), 7.29-7.20(\mathrm{~m}, 4 \mathrm{H}), 7.17-7.15(\mathrm{~m}, 2 \mathrm{H}), 7.05$ (t, $J=8.4 \mathrm{~Hz}, 1 \mathrm{H}), 3.11-3.05$ (m, 2H), 2.53-2.45 (m, 2H), 1.92-1.78 (m, 4H). ${ }^{13} \mathbf{C}$ NMR (100.6 MHz, $\left.\mathrm{CDCl}_{3}\right) \delta 148.1,144.3,128.4,127.4$, 125.6, 125.5, 121.8, 121.6, 121.5, 120.2, 117.9, 76.4, 39.4, 22.9. HRMS-DART $(\mathrm{m} / \mathrm{z}):[\mathrm{M}+\mathrm{H}]^{+}$calcd for $\mathrm{C}_{18} \mathrm{H}_{19} \mathrm{~N}_{2}, 263.1543$; found, 263.1537.

\section{1-(3-Phenyloxetan-3-yl)-1H-indazole (3bl)}

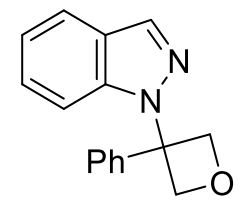

The reaction was carried out according to procedure B. The product $\mathbf{3 b l}$ was purified by flash chromatography on silica gel (100:0-95:5, hexane/EtOAc) (Table 2; $30.2 \mathrm{mg}, 0.12 \mathrm{mmol}, 60 \%$ isolated yield). Yellow oil. IR (neat) 699, 756, 784, 988, 1189, 1383, 1449, 1462, 1515, $1627 \mathrm{~cm}^{-1}$. ${ }^{1} \mathbf{H}$ NMR $\left(400 \mathrm{MHz}, \mathrm{CDCl}_{3}\right) \delta 7.80(\mathrm{~d}, J=10.8 \mathrm{~Hz}, 1 \mathrm{H}), 7.77(\mathrm{~s}, 1 \mathrm{H}), 7.64(\mathrm{~d}, J=8.4 \mathrm{~Hz}, 1 \mathrm{H}), 7.42-$ $7.32(\mathrm{~m}, 4 \mathrm{H}), 7.16-7.10(\mathrm{~m}, 3 \mathrm{H}), 5.70(\mathrm{~d}, J=6.8 \mathrm{~Hz}, 2 \mathrm{H}), 5.32(\mathrm{~d}, J=6.4 \mathrm{~Hz}, 2 \mathrm{H}) .{ }^{13} \mathbf{C}$ NMR $(100.6$ $\left.\mathrm{MHz}_{2} \mathrm{CDCl}_{3}\right) \delta 148.8,140.6,129.0,128.6,126.4,125.4,122.2(\times 2 \mathrm{C}), 121.8,120.2,117.8,82.5$, 67.7. HRMS-DART $(\mathrm{m} / \mathrm{z})$ : $[\mathrm{M}+\mathrm{H}]^{+}$calcd for $\mathrm{C}_{16} \mathrm{H}_{15} \mathrm{~N}_{2} \mathrm{O}, 251.1179$; found, 251.1171.

\section{1-(1-Phenylethyl)-1H-benzo[d][1,2,3]triazole $\quad(3 \mathrm{gm}) \quad$ and 2-(1-Phenylethyl)-2H- benzo[d][1,2,3]triazole (3gm')}<smiles>CC(c1ccccc1)n1nnc2ccccc21</smiles>

$3 g m$<smiles>CC(c1ccccc1)n1nc2ccccc2n1</smiles>

$3 g \mathbf{m}^{\prime}$

The reaction was carried out according to procedure A. The product was purified by flash chromatography on silica gel (100:0-90:10, hexane/EtOAc) (Table 2; 3gm; Yellow oil. 9.1 mg, 0.04 mmol, 20\% isolated yield, 3gm'; Yellow oil. $7.6 \mathrm{mg}, 0.03 \mathrm{mmol}, 17 \%$ isolated yield). The spectrum data of $\mathbf{3 g m}$ or $\mathbf{3 g m}$ ' was consistent with the literature, respectively. ${ }^{9}$ 


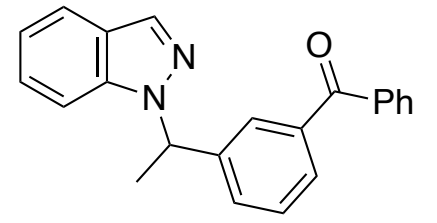

The reaction was carried out according to procedure B. The product $\mathbf{3 b n}$ was purified by flash chromatography on silica gel (100:0-95:5, hexane/EtOAc) (Table 2; $16.3 \mathrm{mg}, 0.05 \mathrm{mmol}, 26 \%$ isolated yield, 36\% NMR yield). Colorless oil. IR (neat) 642, 704, 784, 1155, 1271, 1388, 1446, 1596, 1654, $3058 \mathrm{~cm}^{-1} .{ }^{1} \mathbf{H}$ NMR $\left(400 \mathrm{MHz}, \mathrm{CDCl}_{3}\right) \delta 7.99(\mathrm{~s}, 1 \mathrm{H}), 7.76-7.70(\mathrm{~m}, 5 \mathrm{H}), 7.64(\mathrm{~d}, \mathrm{~J}=8.4 \mathrm{~Hz}$, 1H), $7.56(\mathrm{~m}, 1 \mathrm{H}), 7.46-7.40(\mathrm{~m}, 4 \mathrm{H}), 7.29(\mathrm{~m}, 1 \mathrm{H}), 7.09(\mathrm{t}, \mathrm{J}=7.2 \mathrm{~Hz}, 1 \mathrm{H}), 5.90(\mathrm{q}, \mathrm{J}=7.2 \mathrm{~Hz}, 1 \mathrm{H})$, $2.09(\mathrm{~d}, \mathrm{~J}=7.2 \mathrm{~Hz}, 3 \mathrm{H}) .{ }^{13} \mathbf{C} \mathbf{N M R}\left(100.6 \mathrm{MHz}, \mathrm{CDCl}_{3}\right) \delta 196.2,148.7,141.7,138.0,137.2,132.6$, $130.4,129.8,128.8,128.3,127.9,126.0,121.8,121.4,120.2,117.6,62.3,21.6$. HRMS-DART $(\mathrm{m} / \mathrm{z}):[\mathrm{M}+\mathrm{H}]+$ calcd for $\mathrm{C}_{22} \mathrm{H}_{19} \mathrm{~N}_{2} \mathrm{O}, 327.1492$; found, 327.1493 .

\section{1-[1-(6-Methoxynaphthalen-2-yl)ethyl]-1H-indazole (3bo)}

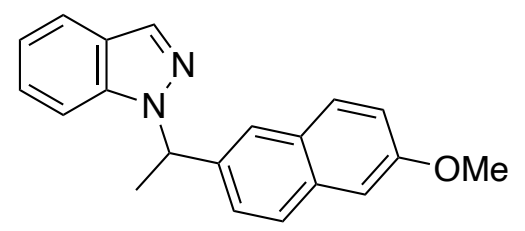

The reaction was carried out according to procedure B. The product $\mathbf{3 b o}$ was purified by flash chromatography on silica gel (100:0-90:10, hexane/EtOAc) (Table 2; $17.9 \mathrm{mg}, 0.06 \mathrm{mmol}, 30 \%$ isolated yield). Pale yellow solid. M.p. $101-104^{\circ} \mathrm{C}$. IR (neat) 475, 753, 853, 1031, 1200, 1266, 1464, 1607, 1634, $2983 \mathrm{~cm}^{-1} .{ }^{1} \mathbf{H}$ NMR $\left(400 \mathrm{MHz}, \mathrm{CDCl}_{3}\right) \delta 8.09(\mathrm{~s}, 1 \mathrm{H}), 7.73(\mathrm{~d}, J=8.0 \mathrm{~Hz}, 1 \mathrm{H}), 7.68-$ $7.63(\mathrm{~m}, 3 \mathrm{H}), 7.33-7.31(\mathrm{~m}, 2 \mathrm{H}), 7.25(\mathrm{~m}, 1 \mathrm{H}), 7.12-7.07(\mathrm{~m}, 3 \mathrm{H}), 5.95(\mathrm{q}, J=6.8 \mathrm{~Hz}, 1 \mathrm{H}), 3.88(\mathrm{~s}$, $3 \mathrm{H}), 2.11(\mathrm{~d}, J=7.2 \mathrm{~Hz}, 3 \mathrm{H}) .{ }^{13} \mathbf{C}$ NMR $\left(100.6 \mathrm{MHz}, \mathrm{CDCl}_{3}\right) \delta 157.7,139.1,137.3,133.9,132.9$, 129.4, 128.6, 127.3, 126.0, 125.0, 124.6, 124.5, 121.0, 120.6, 119.0, 109.6, 105.6, 58.1, 55.3, 21.1. HRMS-DART $(\mathrm{m} / \mathrm{z}):[\mathrm{M}+\mathrm{H}]^{+}$calcd for $\mathrm{C}_{22} \mathrm{H}_{19} \mathrm{~N}_{2} \mathrm{O}, 303.1492$; found, 303.1490 .

\section{1-[1-(Benzyloxy)-2-methylpropyl]-1H-indazole (3bp)}
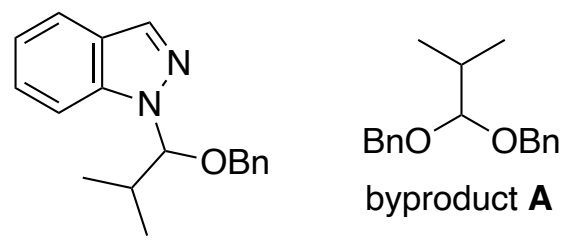

The reaction was carried out according to procedure B. The product $\mathbf{3 b p}$ was purified by flash chromatography on silica gel (Biotage Selekt, 100:0, DCM) (Scheme 2A; $24.7 \mathrm{mg}, 0.09$ mmol, 44\% yield but byproduct A was contaminated.). Yellow oil. IR (neat) 743, 791, 908, 1076, 1171, 1327, 1464, 1615, 2873, $2962 \mathrm{~cm}^{-1} .{ }^{1} \mathbf{H}$ NMR $\left(400 \mathrm{MHz}, \mathrm{CDCl}_{3}\right) \delta 8.04(\mathrm{~s}, 1 \mathrm{H}), 7.76(\mathrm{~d}, J=8.0 \mathrm{~Hz}, 1 \mathrm{H})$, $7.63(\mathrm{~d}, J=8.8 \mathrm{~Hz}, 1 \mathrm{H}), 7.37-7.24(\mathrm{~m}, 6 \mathrm{H}), 7.18(\mathrm{t}, J=7.6 \mathrm{~Hz}, 1 \mathrm{H}), 5.31(\mathrm{~d}, J=9.2 \mathrm{~Hz}, 1 \mathrm{H}), 4.42$ $(\mathrm{d}, J=12.0 \mathrm{~Hz}, 1 \mathrm{H}), 4.36(\mathrm{~d}, J=12.0 \mathrm{~Hz}, 1 \mathrm{H}), 2.65(\mathrm{~m}, 1 \mathrm{H}), 1.18(\mathrm{~d}, J=6.8 \mathrm{~Hz}, 3 \mathrm{H}), 0.56(\mathrm{~d}, J=$ $6.8 \mathrm{~Hz}, 3 \mathrm{H}) .{ }^{13} \mathrm{C}$ NMR $\left(100.6 \mathrm{MHz}, \mathrm{CDCl}_{3}\right) \delta 139.0,137.2,133.5,128.3,128.1,127.8,126.3,124.9$, 
121.1, 121.0, 110.8, 94.8, 70.1, 33.2, 19.3. HRMS-DART $(\mathrm{m} / z):[\mathrm{M}+\mathrm{H}]^{+}$calcd for $\mathrm{C}_{18} \mathrm{H}_{21} \mathrm{~N}_{2} \mathrm{O}$, 281.1648 ; found,281.1641.

\section{5-\{[7-(6-Bromo-1H-indazol-1-yl)-7-methyloctyl]oxy\}-2-(2,6-dioxopiperidin-3-yl)isoindoline-}

\section{1,3-dione (3aq)}<smiles>CC(C)(CCCCCCOc1ccc2c(c1)C(=O)N(C1CCC(=O)NC1=O)C2=O)n1ncc2ccc(Br)cc21</smiles>

The reaction was carried out according to procedure $\mathrm{B}(0.1 \mathrm{mmol}$ scale). The product 3 aq was purified by flash chromatography on silica gel (Biotage Selekt, 80:20-50:50, hexane/EtOAc) (Scheme 2C; $36.9 \mathrm{mg}, 0.06 \mathrm{mmol}, 31 \%$ yield.). Colorless oil. IR (neat) 750, 920, 1113, 1198, 1258, 1288, 1380, 1450, 1490, 1619, 1711, 1773, $2939 \mathrm{~cm}^{-1} .{ }^{1} \mathbf{H}$ NMR (400 MHz, $\left.\mathrm{CDCl}_{3}\right) \delta 8.14$ (br s, $\left.1 \mathrm{H}\right)$, 7.99 (s, 1H), $7.90(\mathrm{~s}, 1 \mathrm{H}), 7.75(\mathrm{~d}, J=8.4 \mathrm{~Hz}, 1 \mathrm{H}), 7.52$ (d, J=8.8 Hz, 1H), 7.29 (s, 1H), 7.14 (s, 1H), $7.12(\mathrm{~s}, 1 \mathrm{H}), 4.96(\mathrm{dd}, J=11.2 \mathrm{~Hz}, 4.8 \mathrm{~Hz}, 1 \mathrm{H}), 4.00(\mathrm{t}, J=6.0 \mathrm{~Hz}, 2 \mathrm{H}), 2.92-2.70(\mathrm{~m}, 3 \mathrm{H})$, $2.14(\mathrm{~m}, 1 \mathrm{H}), 2.04-2.00(\mathrm{~m}, 2 \mathrm{H}), 1.77-1.65(\mathrm{~m}, 8 \mathrm{H}), 1.39-1.27(\mathrm{~m}, 4 \mathrm{H}), 1.04-1.02(\mathrm{~m}, 2 \mathrm{H}) .{ }^{13} \mathrm{C}$ NMR (100.6 MHz, $\left.\mathrm{CDCl}_{3}\right) \delta 170.8,168.0,167.2,167.0,164.5,149.0,134.3,125.5,125.0,123.2$, 121.6, 120.7, 120.6, 120.0, 119.6, 119.5, 108.8, 68.9, 62.9, 49.3, 42.7, 31.4, 29.2, 28.7, 27.9, 25.6, 23.6, 22.7. HRMS-DART $(\mathrm{m} / \mathrm{z})$ : $[\mathrm{M}+\mathrm{H}]^{+}$calcd for $\mathrm{C}_{29} \mathrm{H}_{32} \mathrm{BrN}_{4} \mathrm{O}_{5}, 595.1551$; found,595.1552.

\section{Results with Other Class of Photoredox Catalysts}

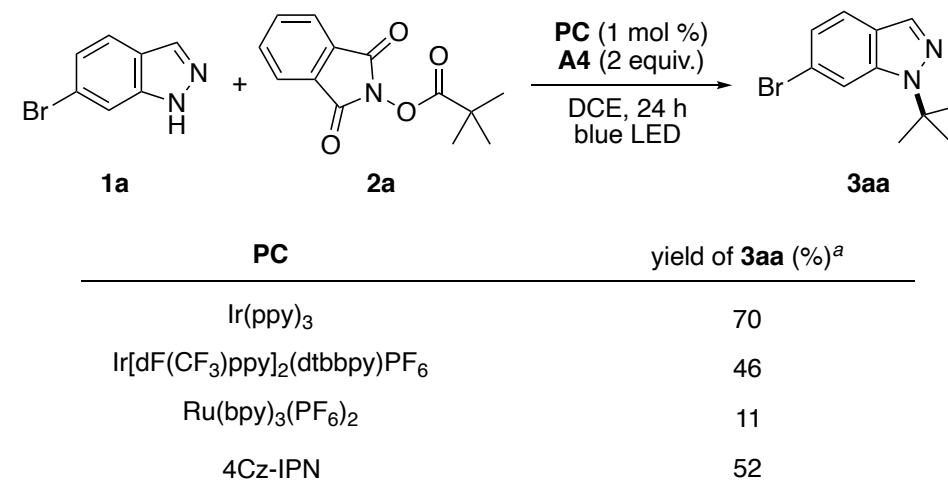

Figure S3. Results with Other Class of Photoredox Catalysts

8. Effect of Collidine $\cdot \mathrm{HBF}_{4}$

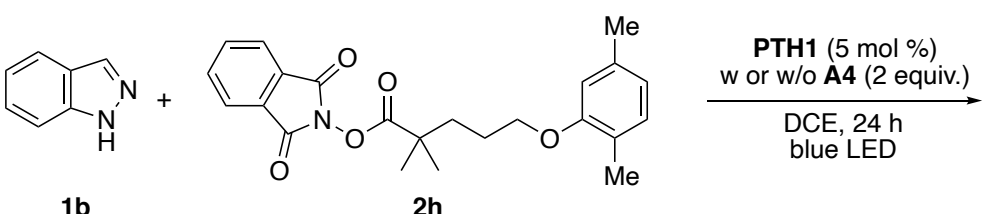

$1 b$

2h

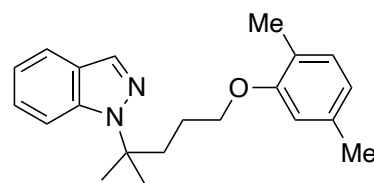

with A4 without A4 3bh, 52\% 3bh, trace

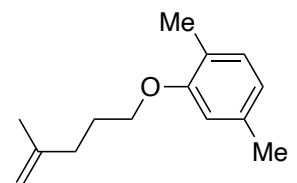

2h-1, 40\% 2h-1, 55\%

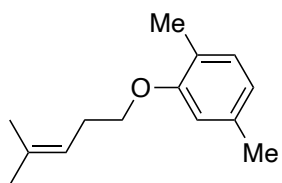

2h-1, 21\% 2h-1, 18\%

Figure S4. Effect of Collidine $\cdot \mathrm{HBF}_{4}$ 


\section{Cyclic Voltammetry (CV) Experiments}

$\mathrm{CV}$ measurements were carried out under nitrogen atmosphere in acetonitrile solutions with $0.1 \mathrm{M}$ of tetrabutylammonium hexafluorophosphate $\left(\mathrm{Bu}_{4} \mathrm{NPF}_{4}\right)$ as a supporting electrolyte. Measurements were made with a glassy carbon electrode ( $\mathrm{area}=0.07 \mathrm{~cm}^{2}$ ), an $\mathrm{Ag} / \mathrm{AgCl}$ reference electrode, and a Pt wire counter electrode. The concentration of the sample solution was fixed at $10 \mathrm{mM}$ and the sweep rates were set to $100 \mathrm{mV} / \mathrm{s}$.

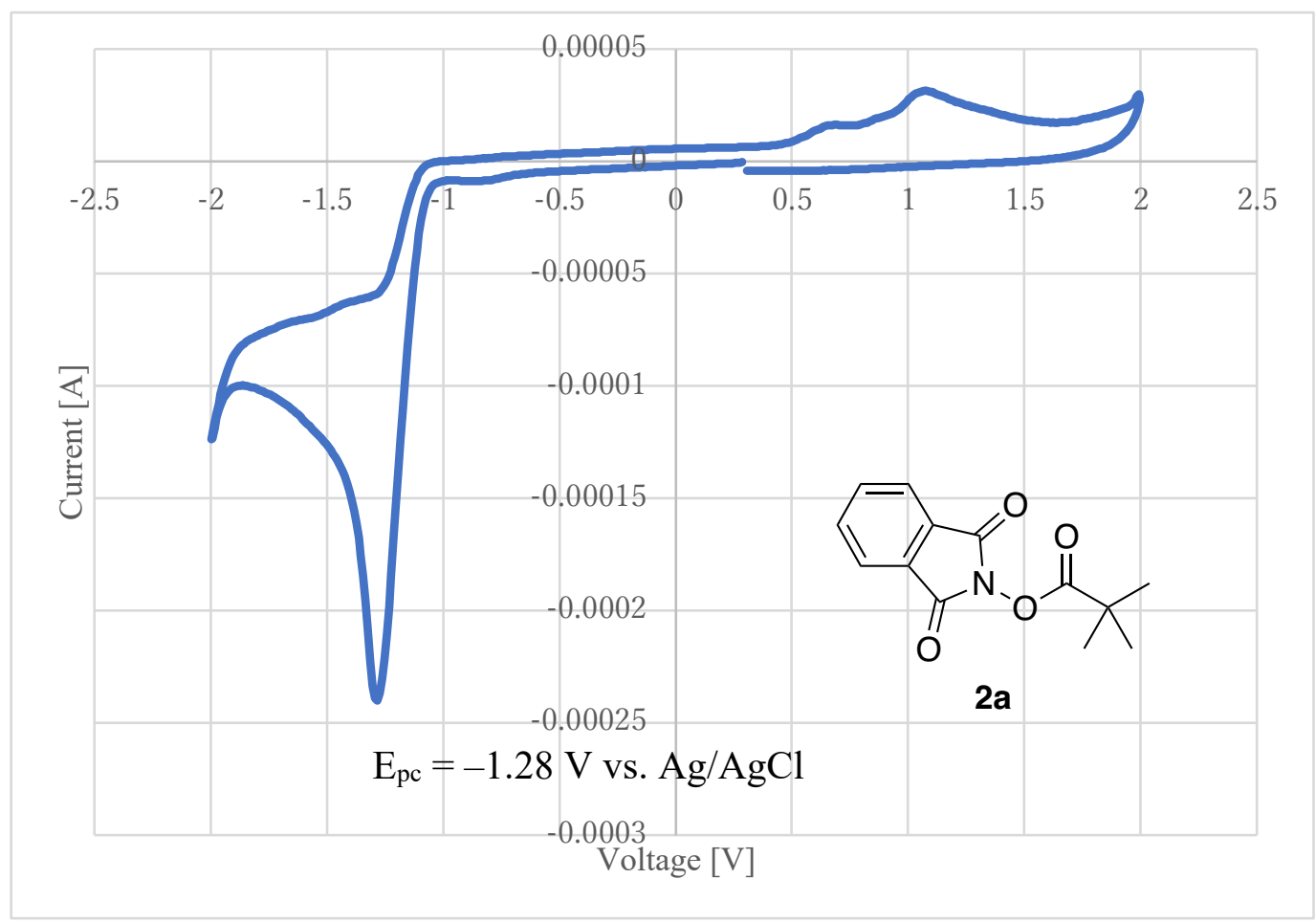

Figure S5. Cyclic voltammogram of $\mathbf{2 a}$.

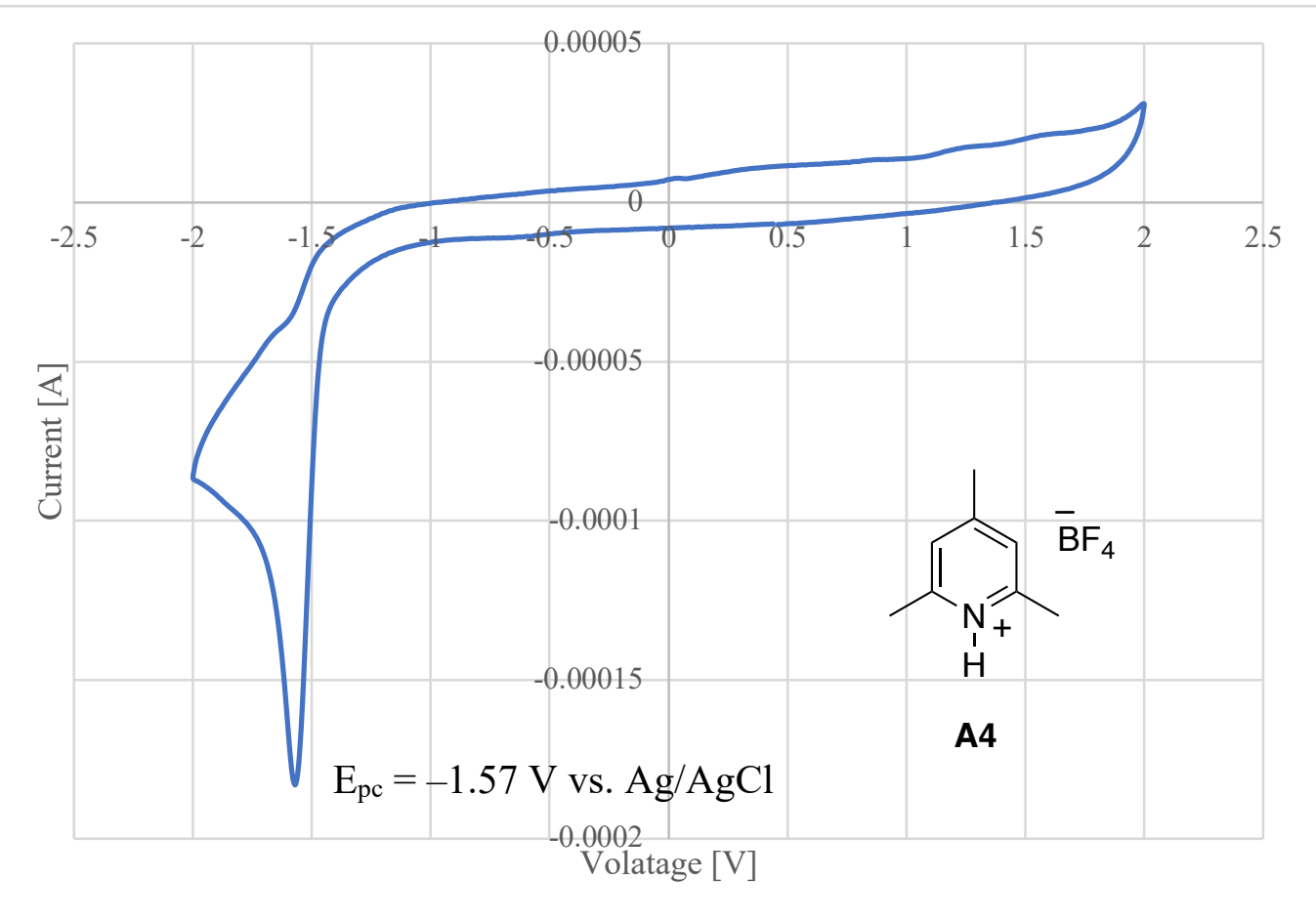

Figure S6. Cyclic voltammogram of A4. 


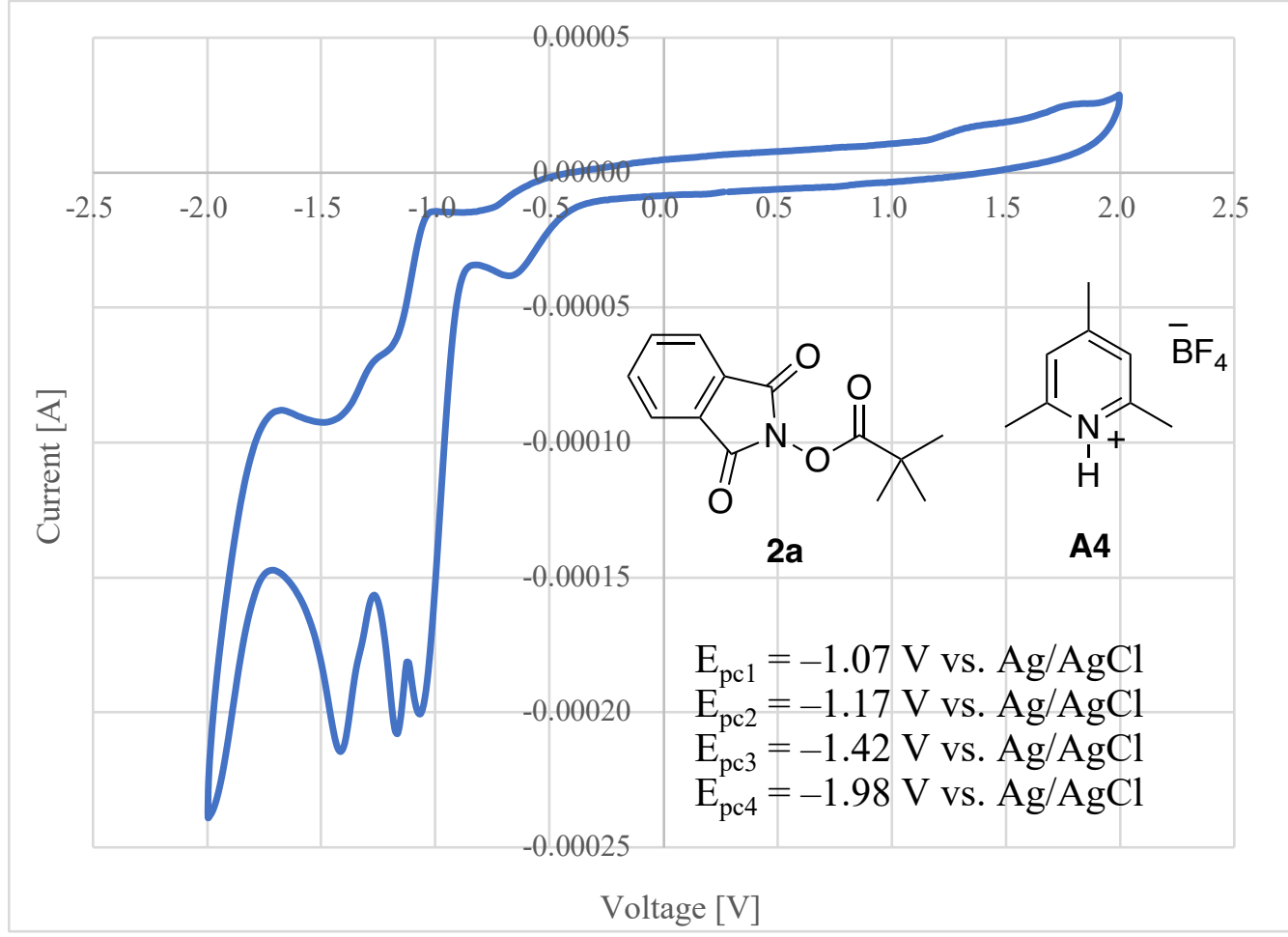

Figure S7. Cyclic voltammogram of $2 \mathbf{a}$ and $4 \mathbf{a}(1: 1)$.

\section{UV-Vis Spectra of Reaction Mixtures}

UV-Vis spectra of the reation mixture were measured under identical concentration to the catalytic reaction conditions [redox active ester $2 \mathbf{2 a}(0.2 \mathrm{mmol})$, PTH1 $(5 \mathrm{~mol} \%)$ and collidine $\cdot \mathrm{HBF}_{4}(0.2$ $\mathrm{mmol})$ in 1,2-dichloroethane $(3 \mathrm{~mL})$ solution].

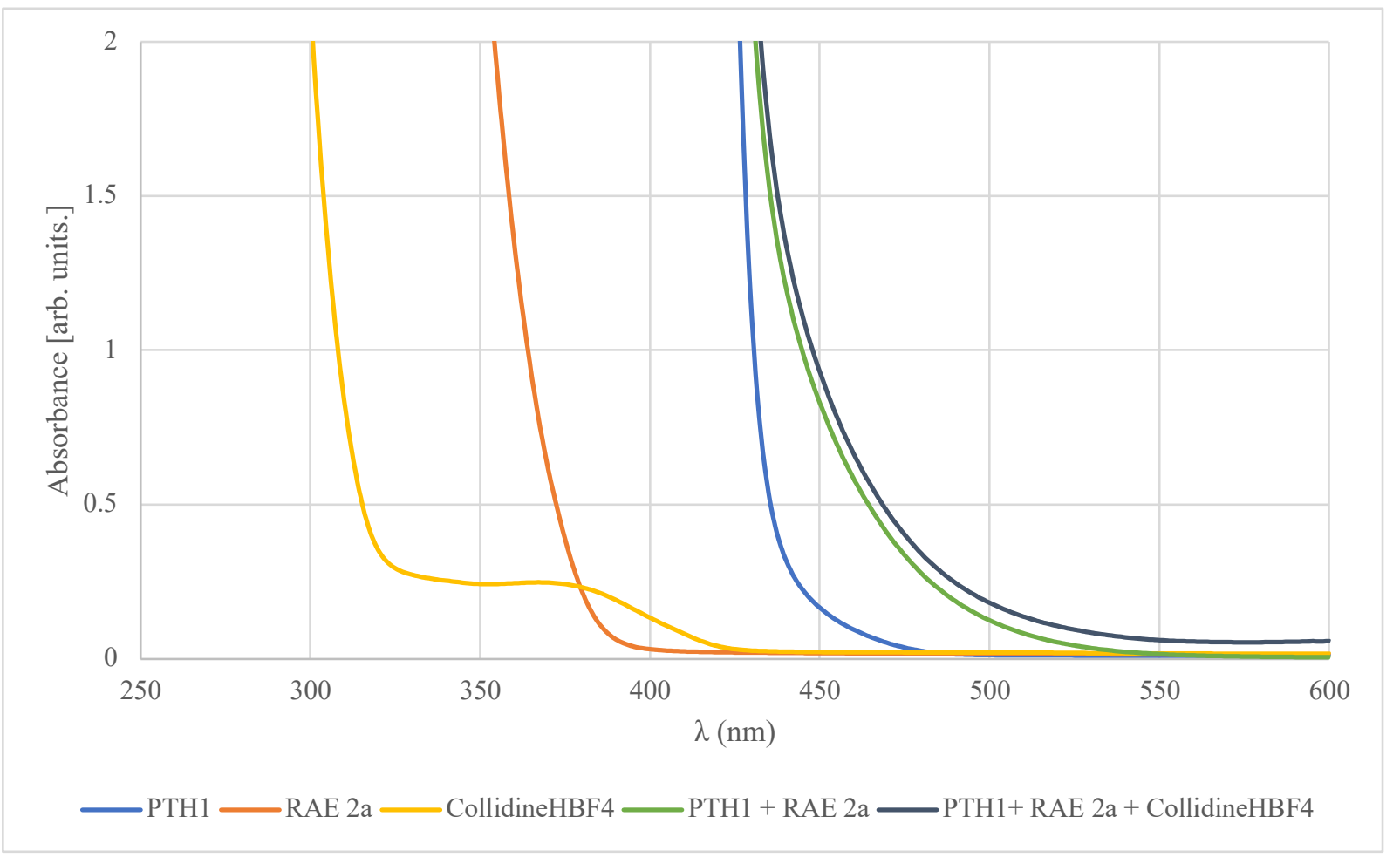

Figure S8. UV-Vis spectra of reaction mixtures. 


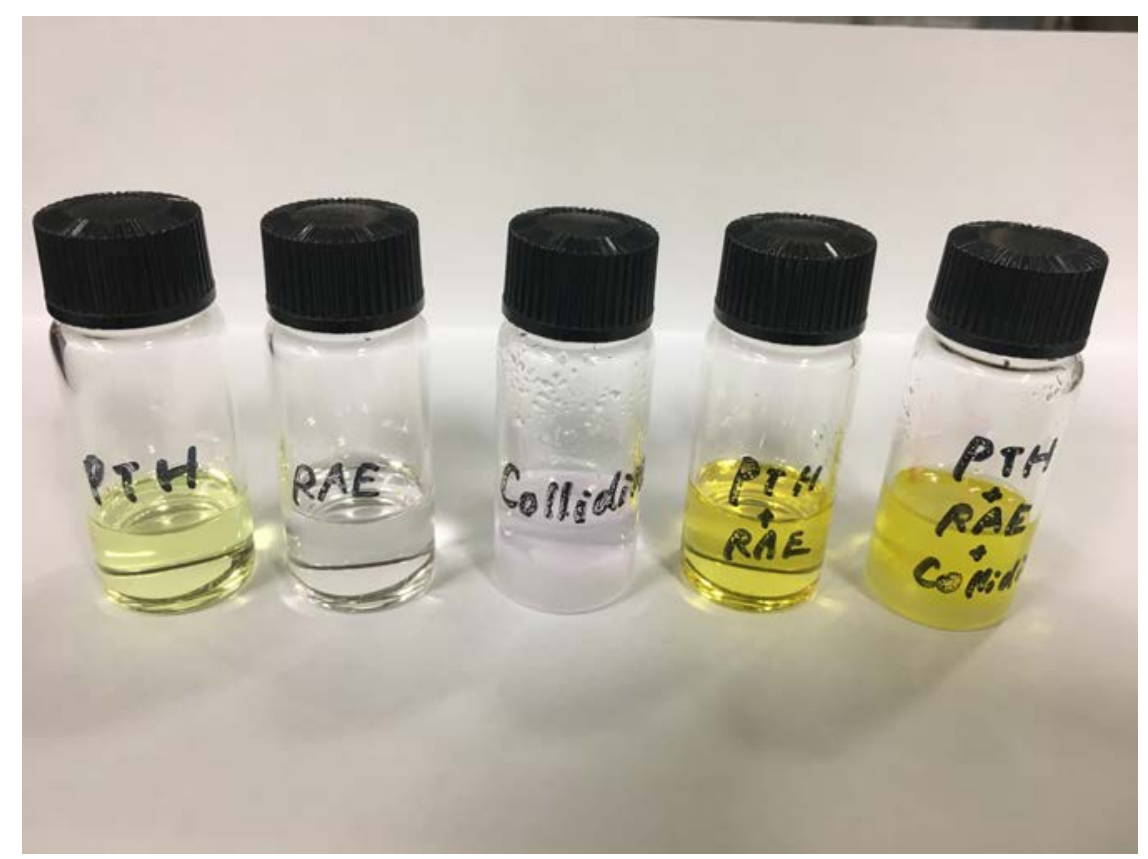

Figure S9. Color of solutions.

\section{- Supplementary References}

(1) Qin, T.; Cornella, J.; Li, C.; Malins, L. R.; Edwards, J. T.; Kawamura, S.; Maxwell, B. D.; Eastgate, M. D.; Baran, P. S. Science 2016, 352, 801.

(2) Treat, N. J.; Sprafke, H.; Kramer, J. W.; Clark, P. G.; Barton, B. E.; Read de Alaniz, J.; Fors, B. P.; Hawker, C. J. J. Am. Chem. Soc. 2014, 136, 16096.

(3) Dadashi-Silab, S.; Pan, X.; Matyjaszewski, K. Chem. Eur. J. 2017, 23, 5972.

(4) Crivello, J. V. J. Polym. Sci., Part A: Polym. Chem. 2008, 46, 3820.

(5) Ohmori, H.; Takanami, T.; Shimada, H.; Masui, M. Chem. Phama. Bull. 1987, 35, 2558.

(6) Le, Z.-G.; Chen, Z.-C. Hu, Y.; Zheng, Q.-G. Heterocycles, 2004, 63, 1077.

(7) Sheng, T.; Zhang, H.-J.; Shang, M.; He, C.; Vantourout, J. C.; Baran, P. S. Org. Lett. 2020, 22,7594 .

(8) Luo, Z.; Jiang, Z.; Jiang, W.; Lin, D. J. Org. Chem. 2018, 83, 3710.

(9) Song, C.; Dong, X.; Yi, H.; Chiang, C.-W.; Lei, A. ACS Catal. 2018, 8, 2195. 


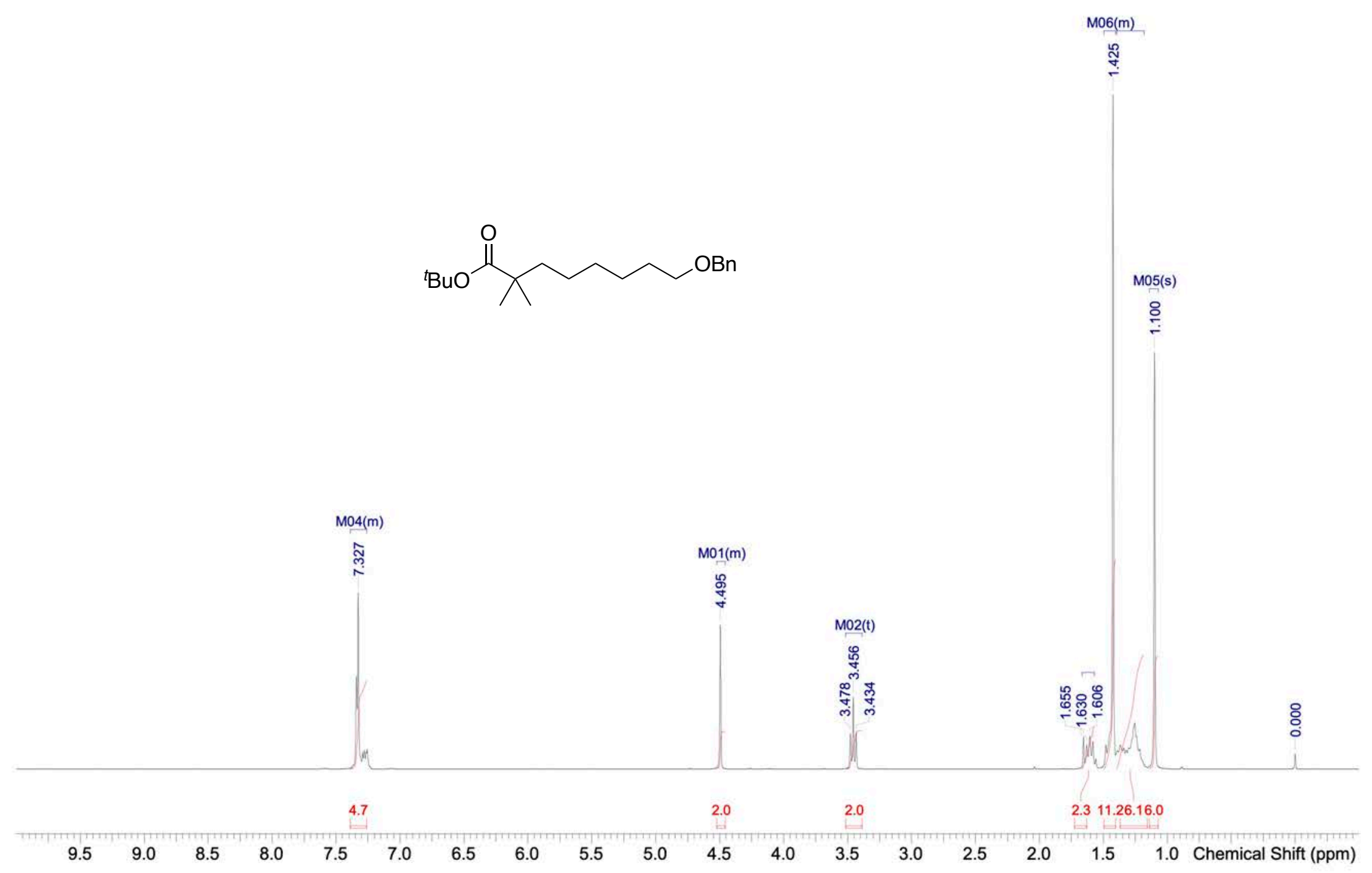

${ }^{1} \mathrm{H}$ NMR spectrum of $\mathbf{S 2}\left(300 \mathrm{MHz}, \mathrm{CDCl}_{3}\right)$ 


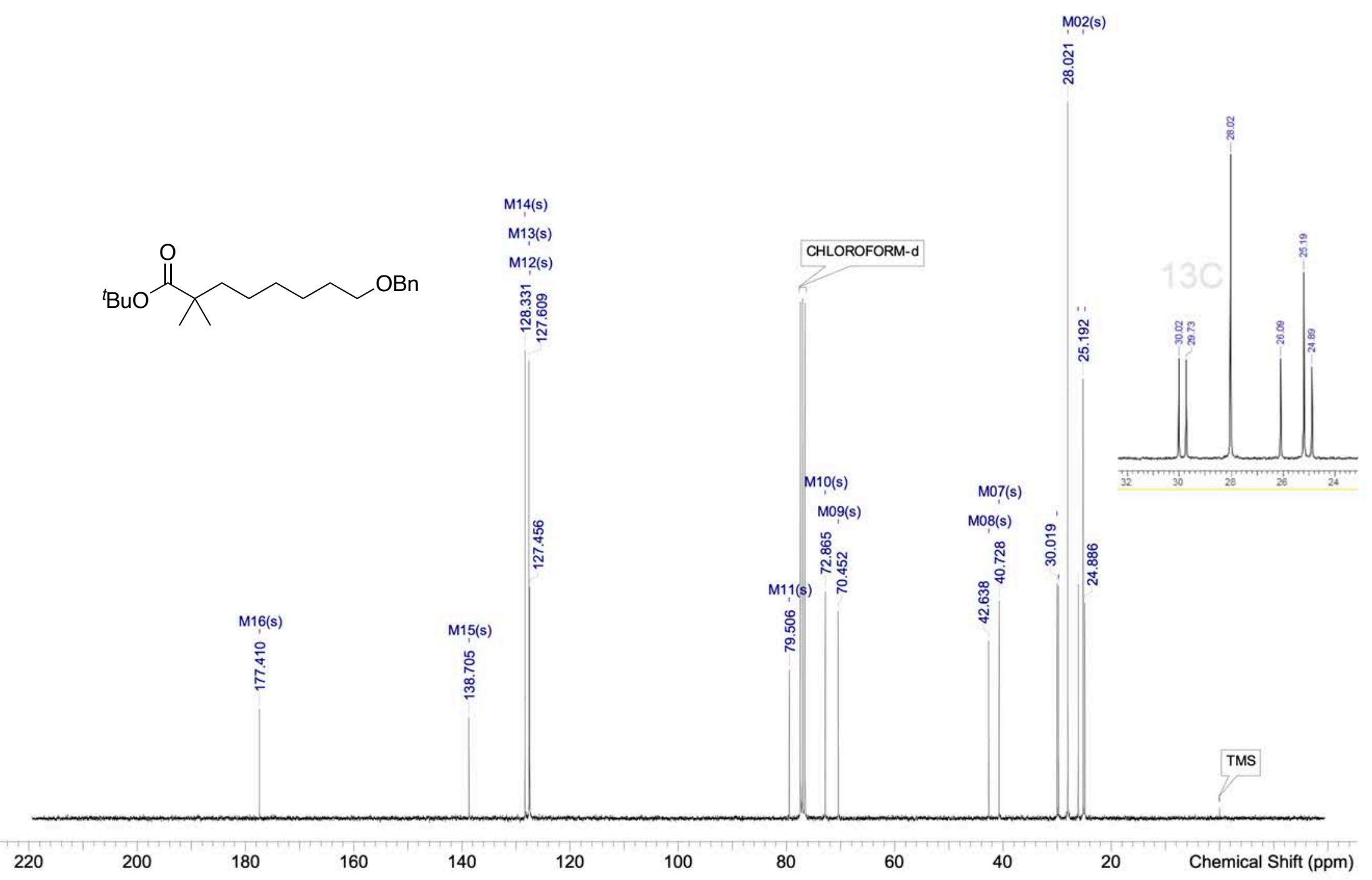

${ }^{13} \mathrm{C}$ NMR spectrum of $\mathbf{S 2}\left(75.0 \mathrm{MHz}, \mathrm{CDCl}_{3}\right)$ 


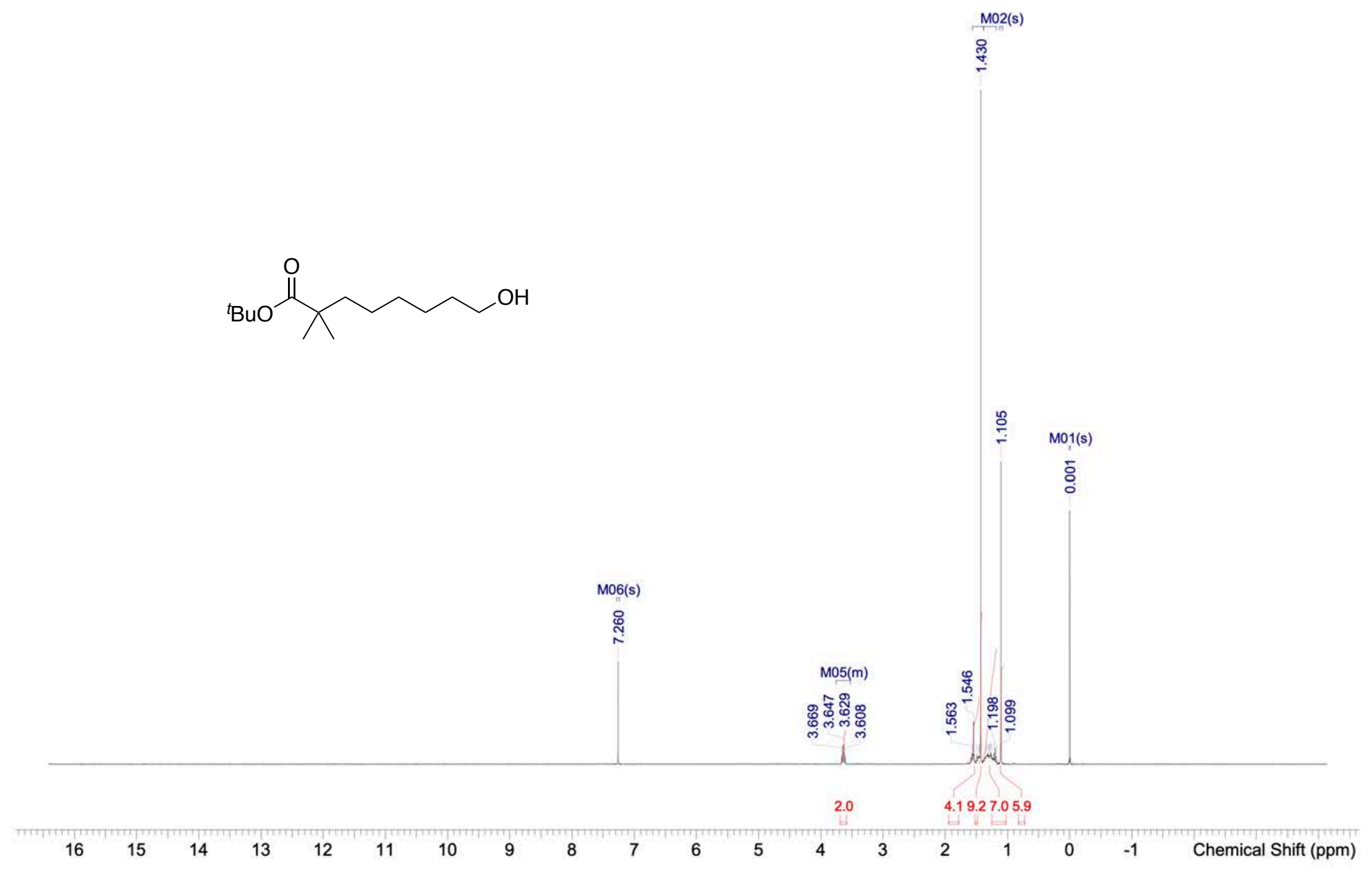

${ }^{1} \mathrm{H}$ NMR spectrum of $\mathbf{S 3}\left(300 \mathrm{MHz}, \mathrm{CDCl}_{3}\right)$ 


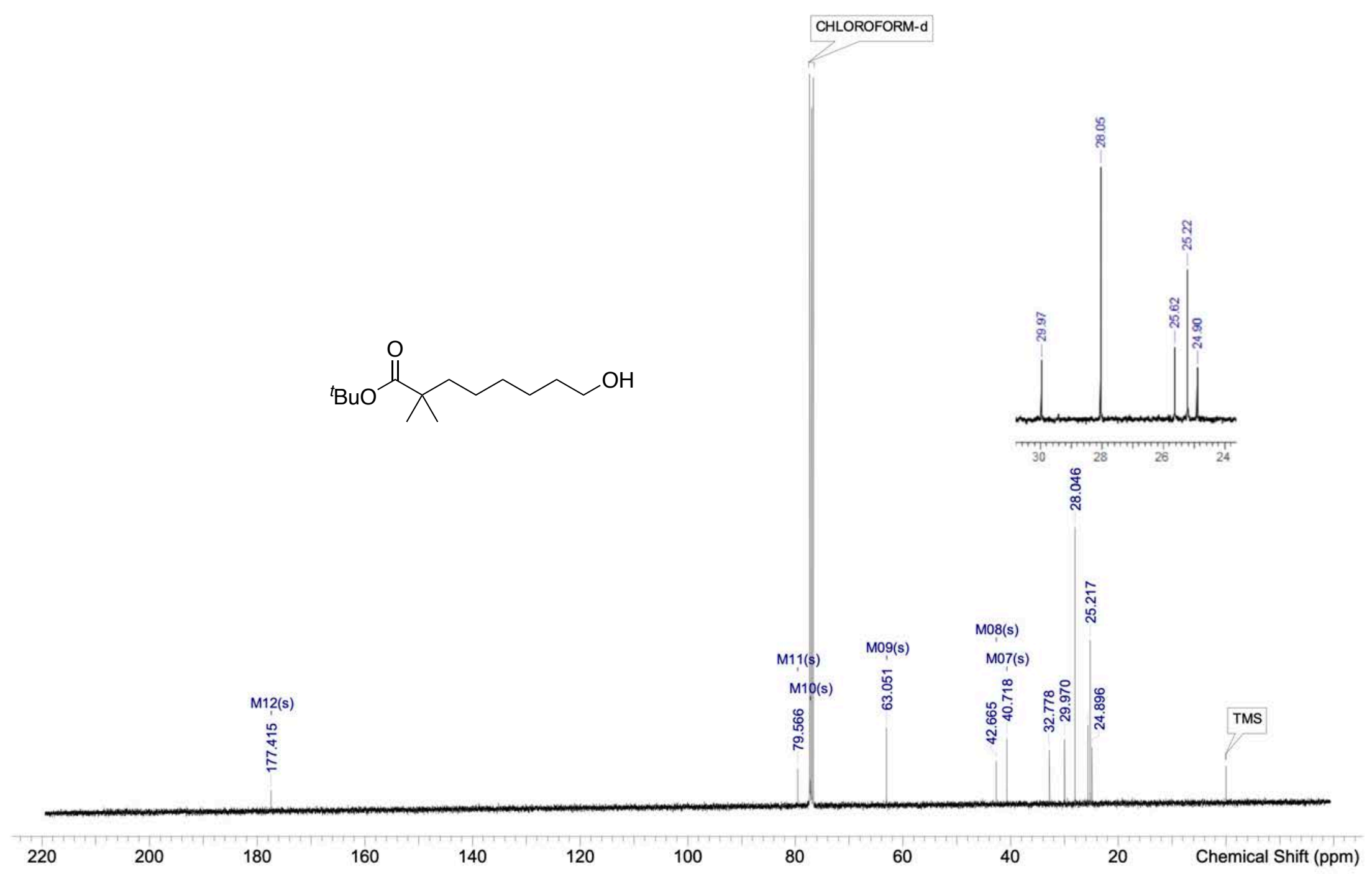

${ }^{13} \mathrm{C}$ NMR spectrum of $\mathbf{S 3}\left(75.0 \mathrm{MHz}, \mathrm{CDCl}_{3}\right)$ 


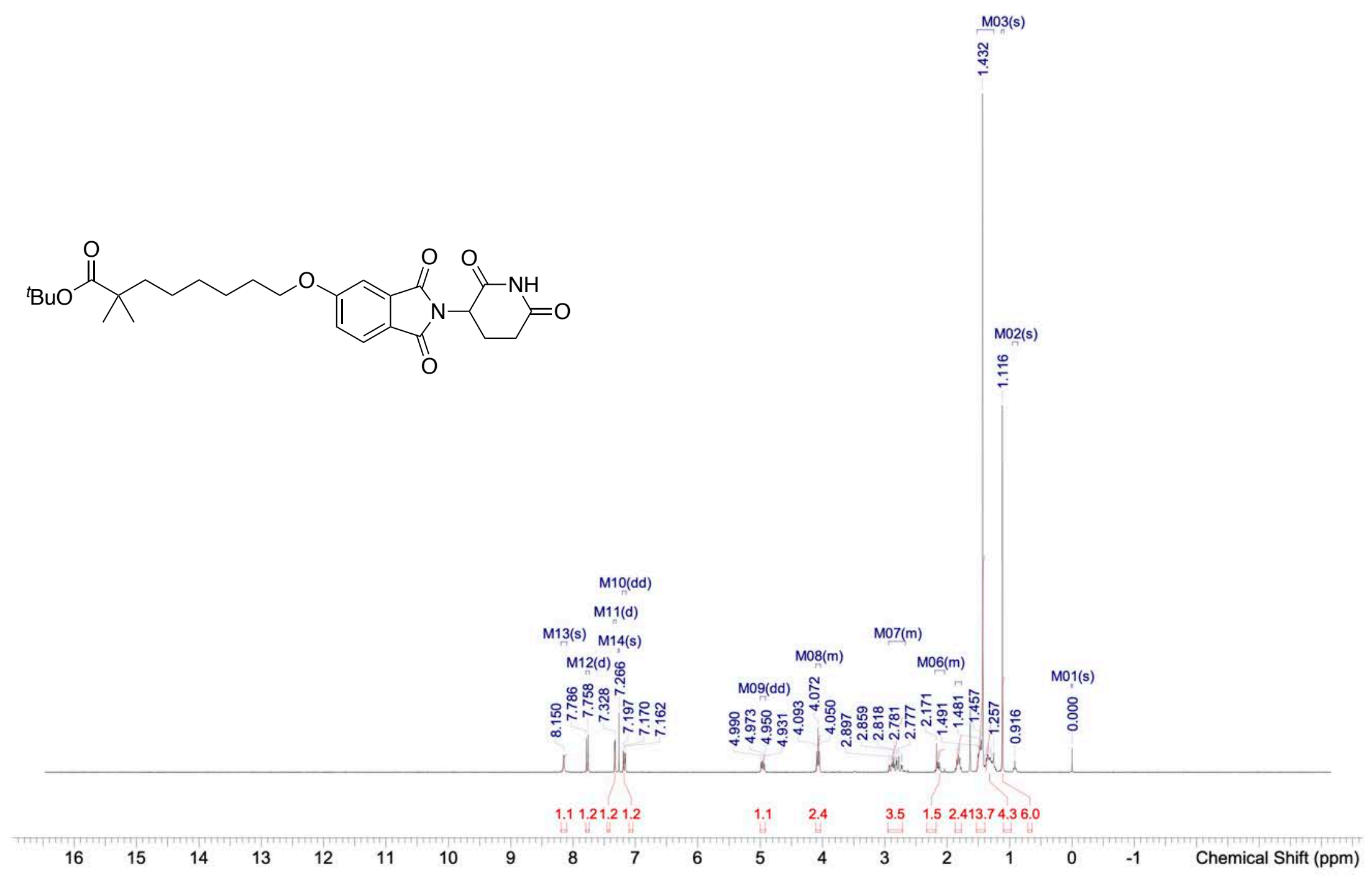

${ }^{1} \mathrm{H}$ NMR spectrum of $\mathbf{S 4}\left(300 \mathrm{MHz}, \mathrm{CDCl}_{3}\right)$ 


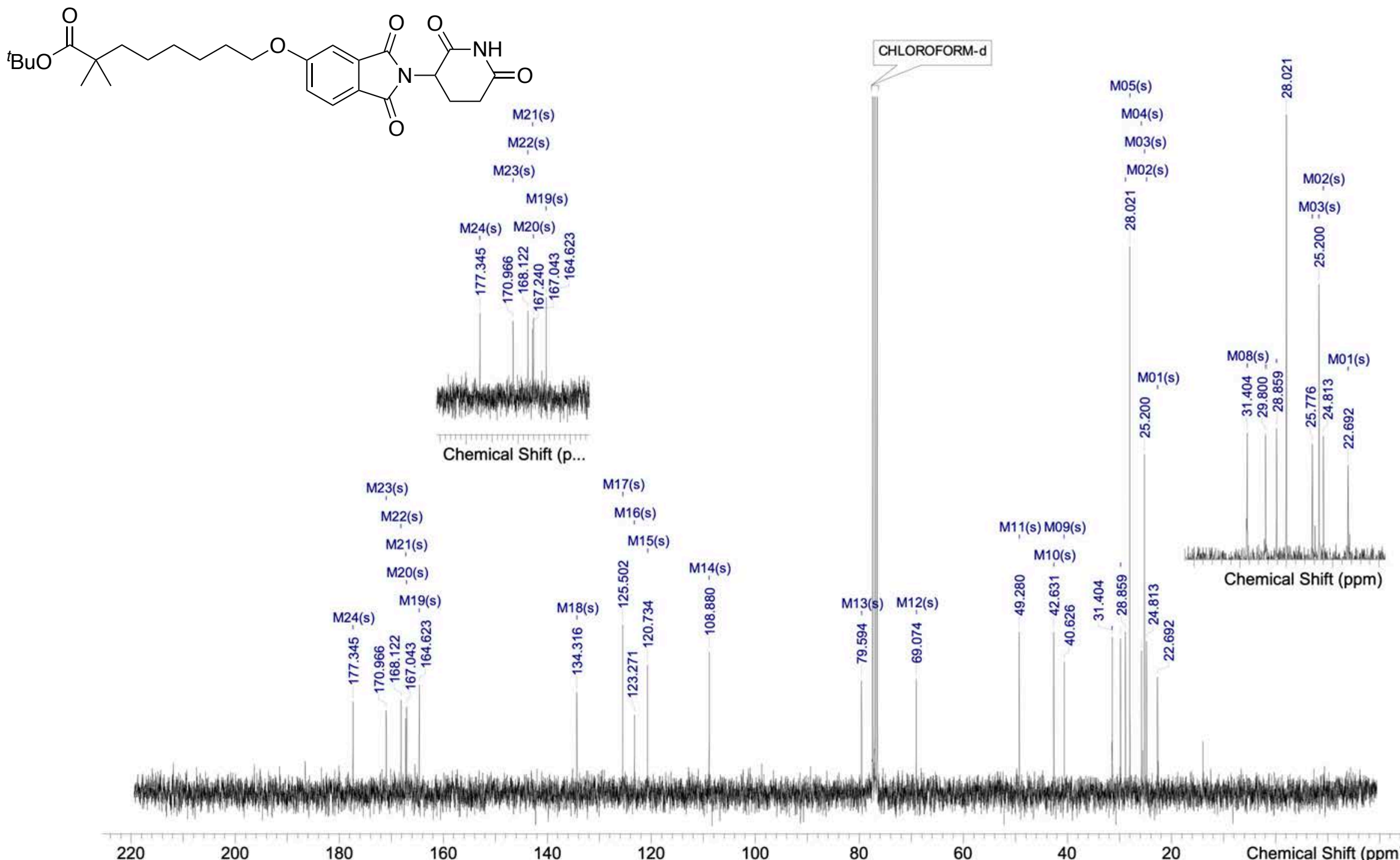

${ }^{13} \mathrm{C}$ NMR spectrum of $\mathbf{S 4}\left(75.0 \mathrm{MHz}, \mathrm{CDCl}_{3}\right)$ 


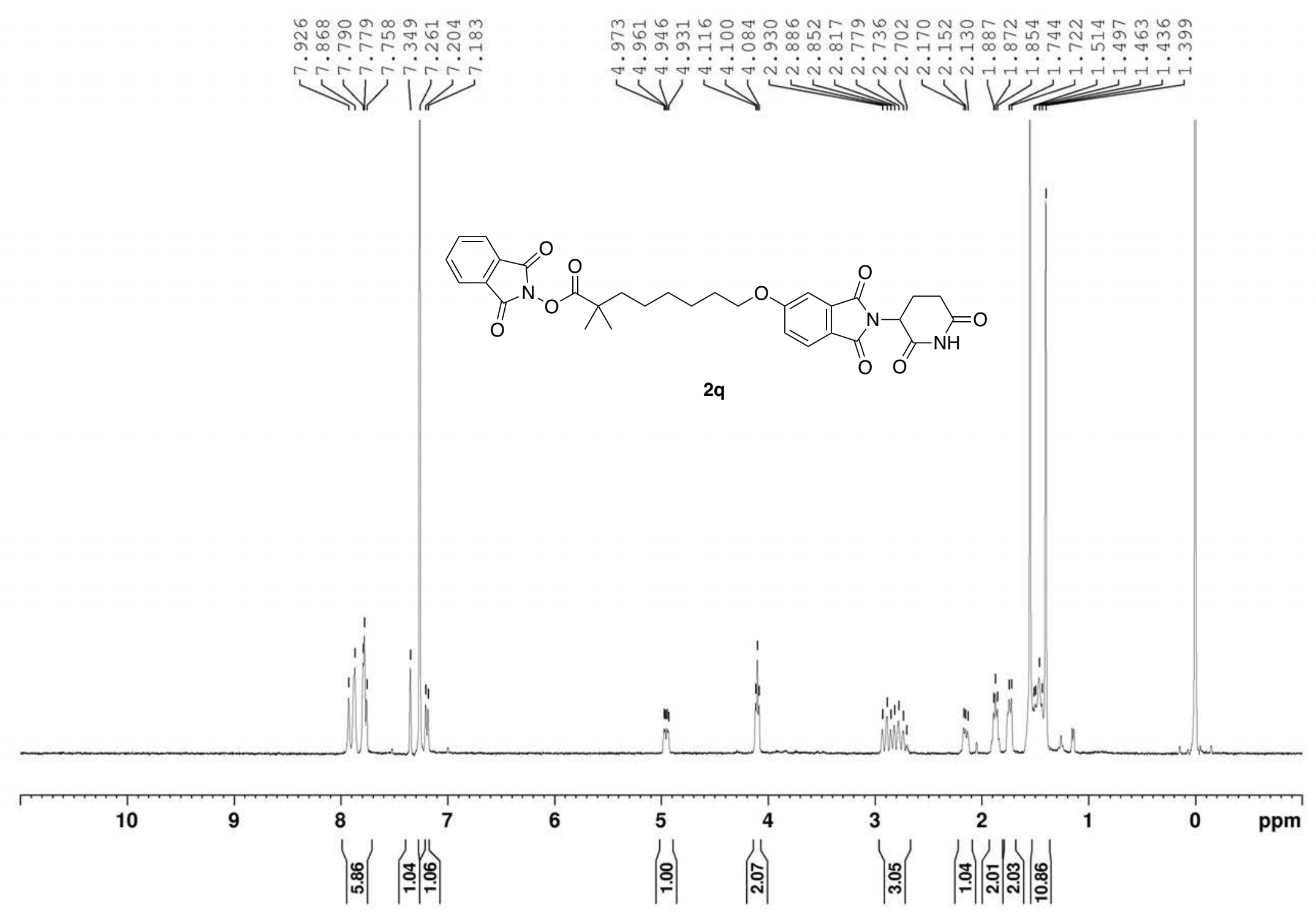

${ }^{1} \mathrm{H}$ NMR spectrum of $\mathbf{2 q}\left(400 \mathrm{MHz}, \mathrm{CDCl}_{3}\right)$ 


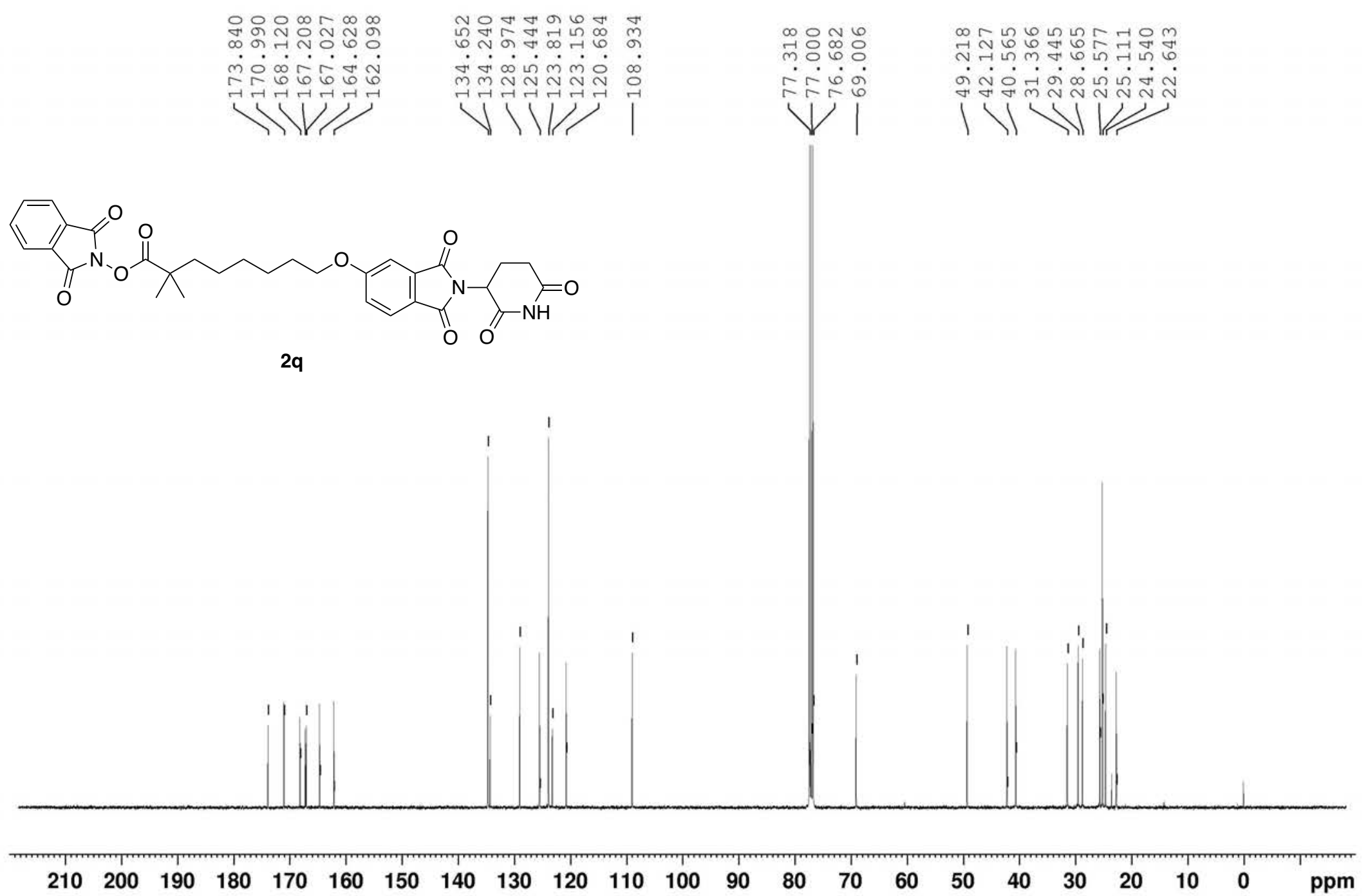

${ }^{13} \mathrm{C}$ NMR spectrum of $\mathbf{2 q}\left(100.6 \mathrm{MHz}, \mathrm{CDCl}_{3}\right)$ 


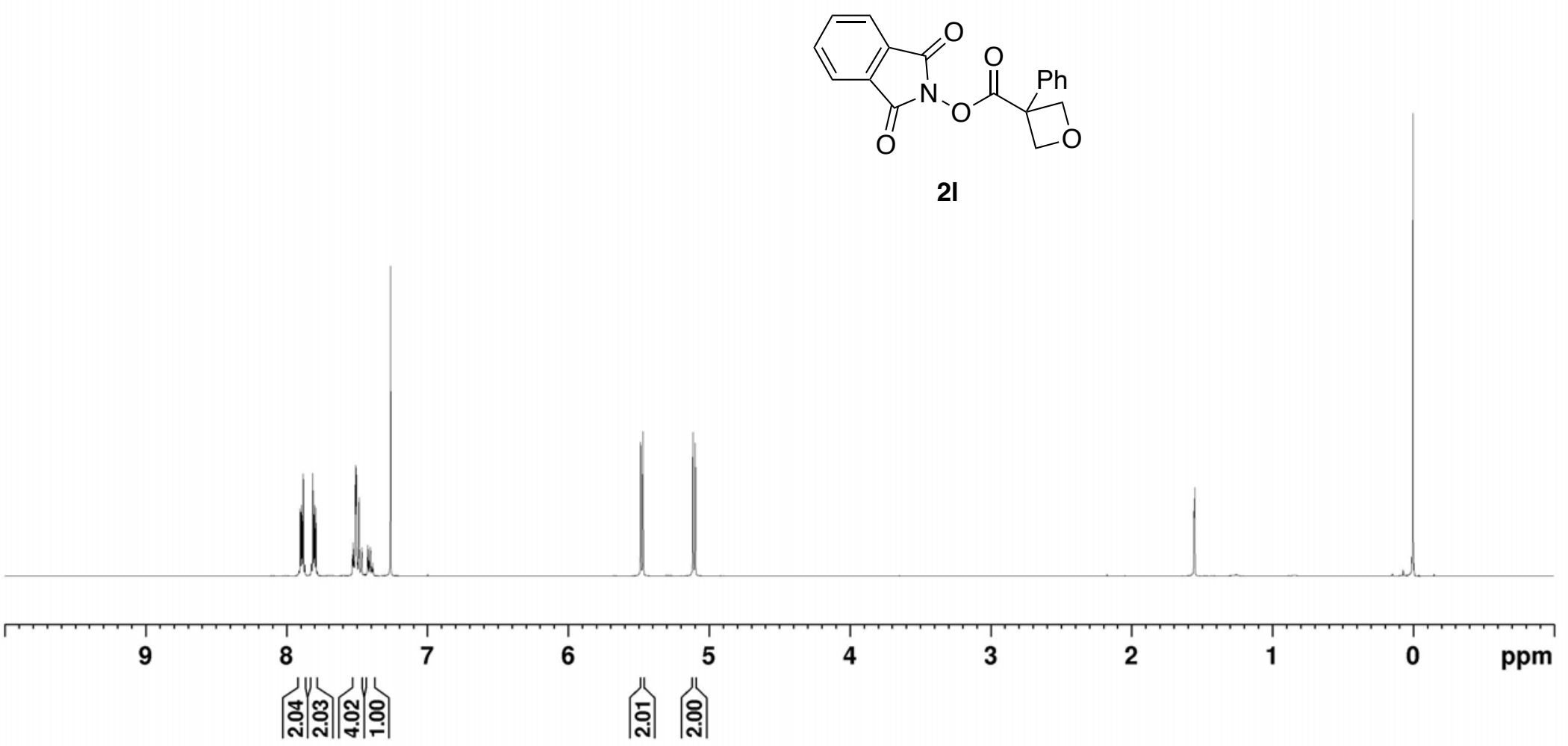

${ }^{1} \mathrm{H}$ NMR spectrum of $2 \mathbf{l}\left(400 \mathrm{MHz}, \mathrm{CDCl}_{3}\right)$ 


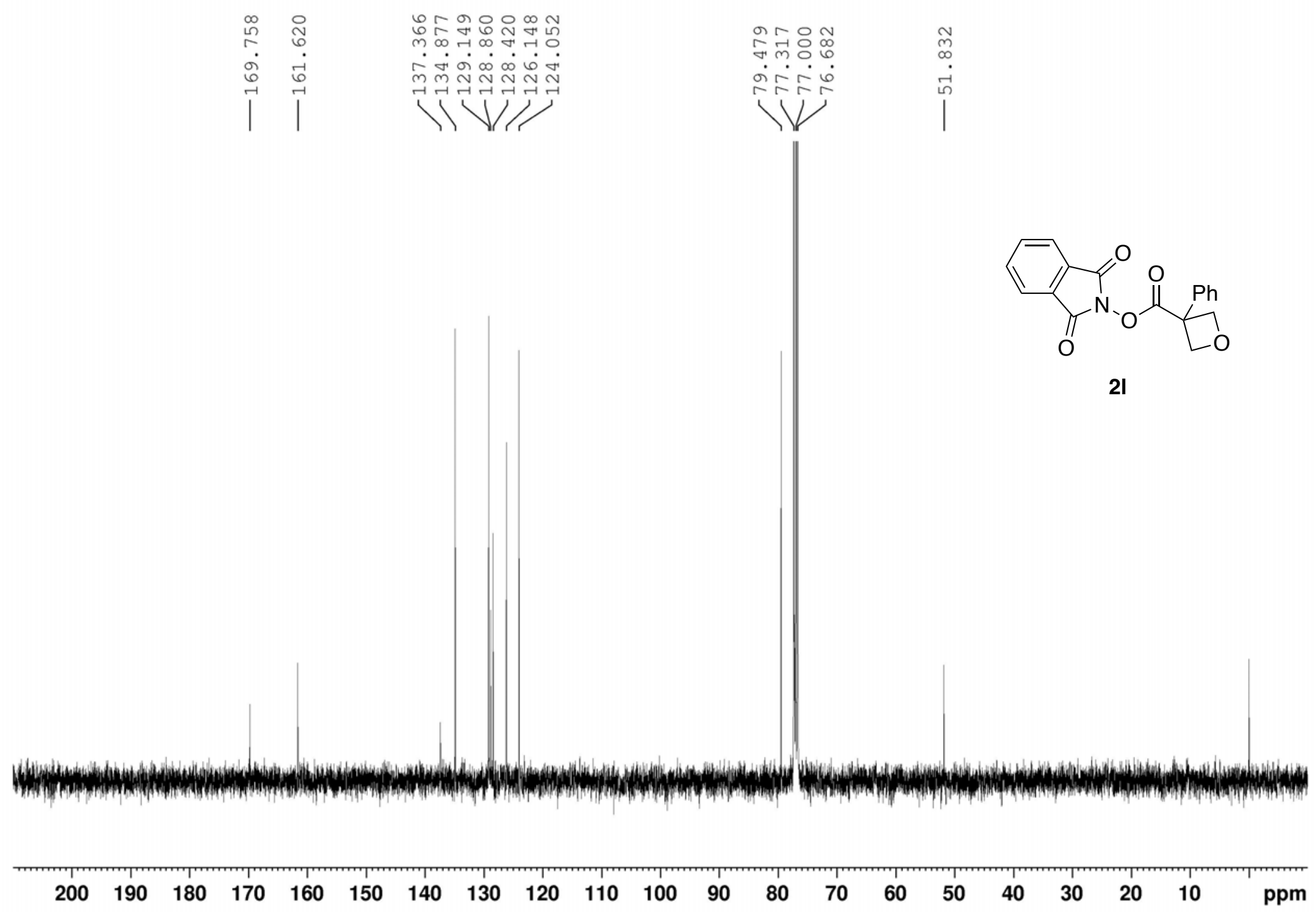

${ }^{13} \mathrm{C}$ NMR spectrum of $2 \mathbf{2}\left(100.6 \mathrm{MHz}, \mathrm{CDCl}_{3}\right)$ 


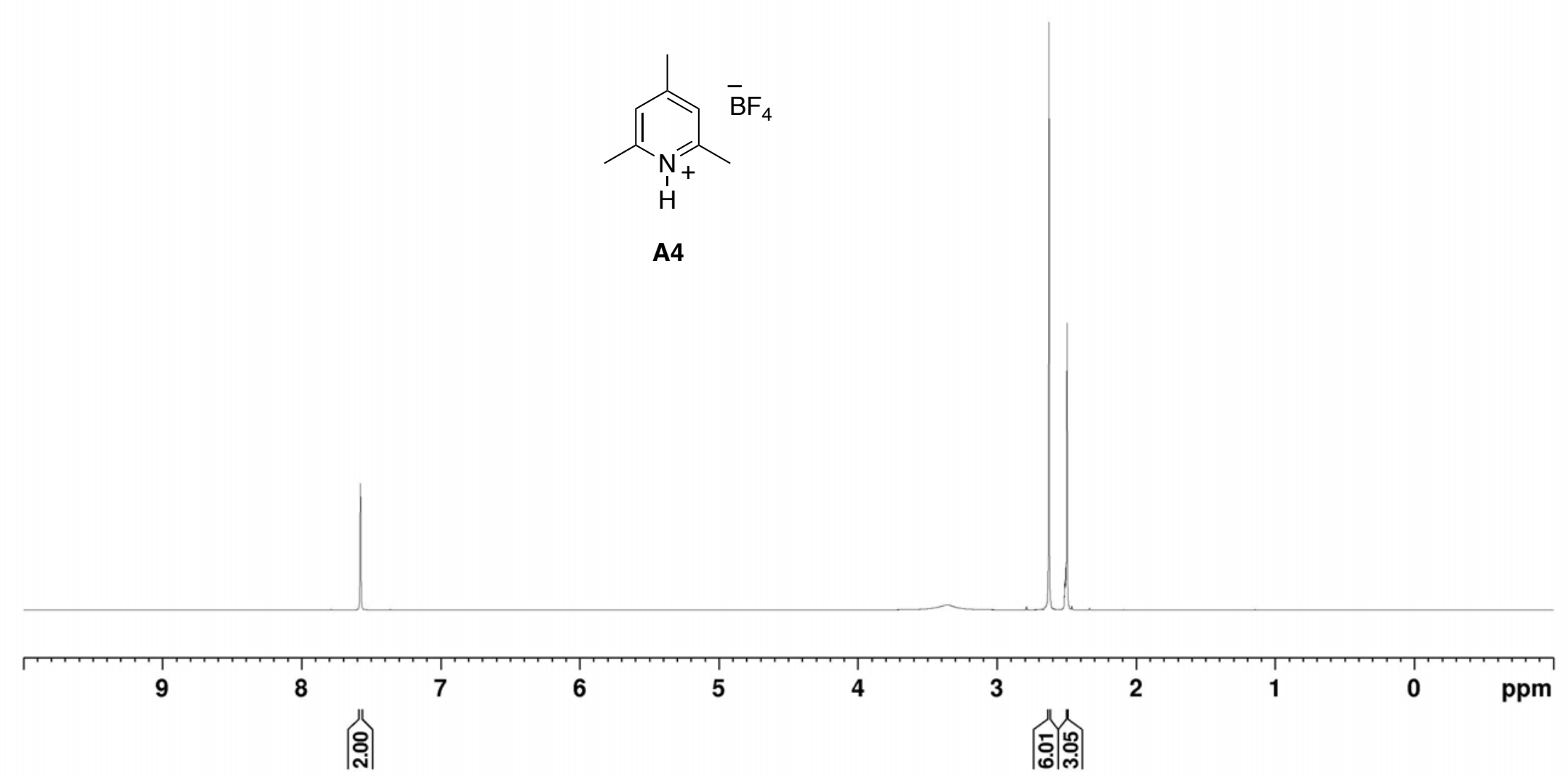

${ }^{1} \mathrm{H}$ NMR spectrum of $\mathbf{A 4}\left(400 \mathrm{MHz}, \mathrm{DMSO}-d_{6}\right)$ 

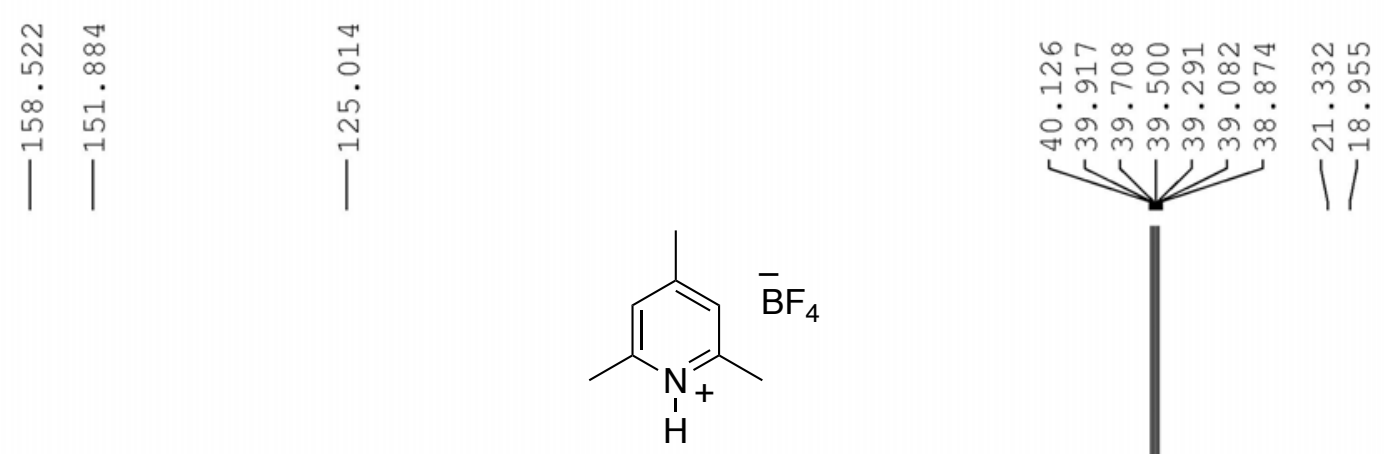

A4

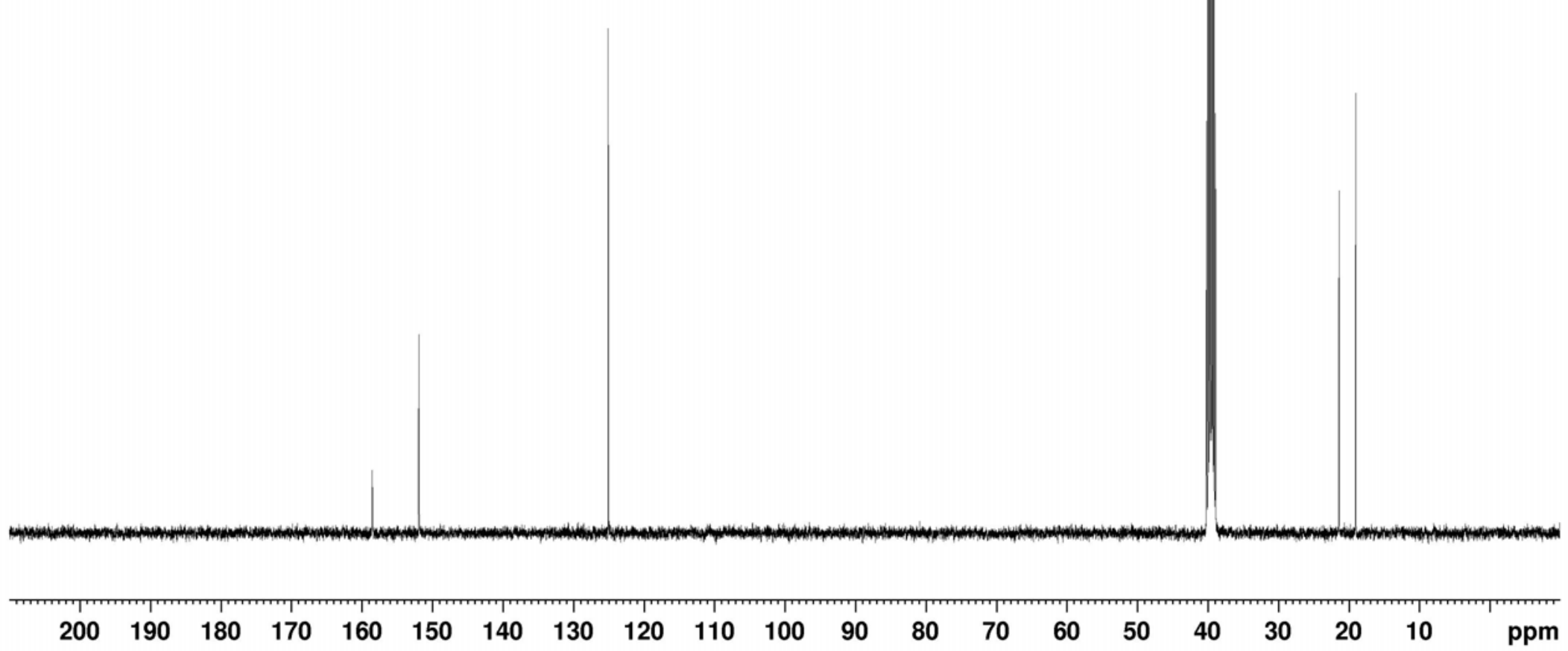

${ }^{13} \mathrm{C}$ NMR spectrum of $\mathbf{A 4}\left(100.6 \mathrm{MHz}\right.$, DMSO- $\left.d_{6}\right)$ 


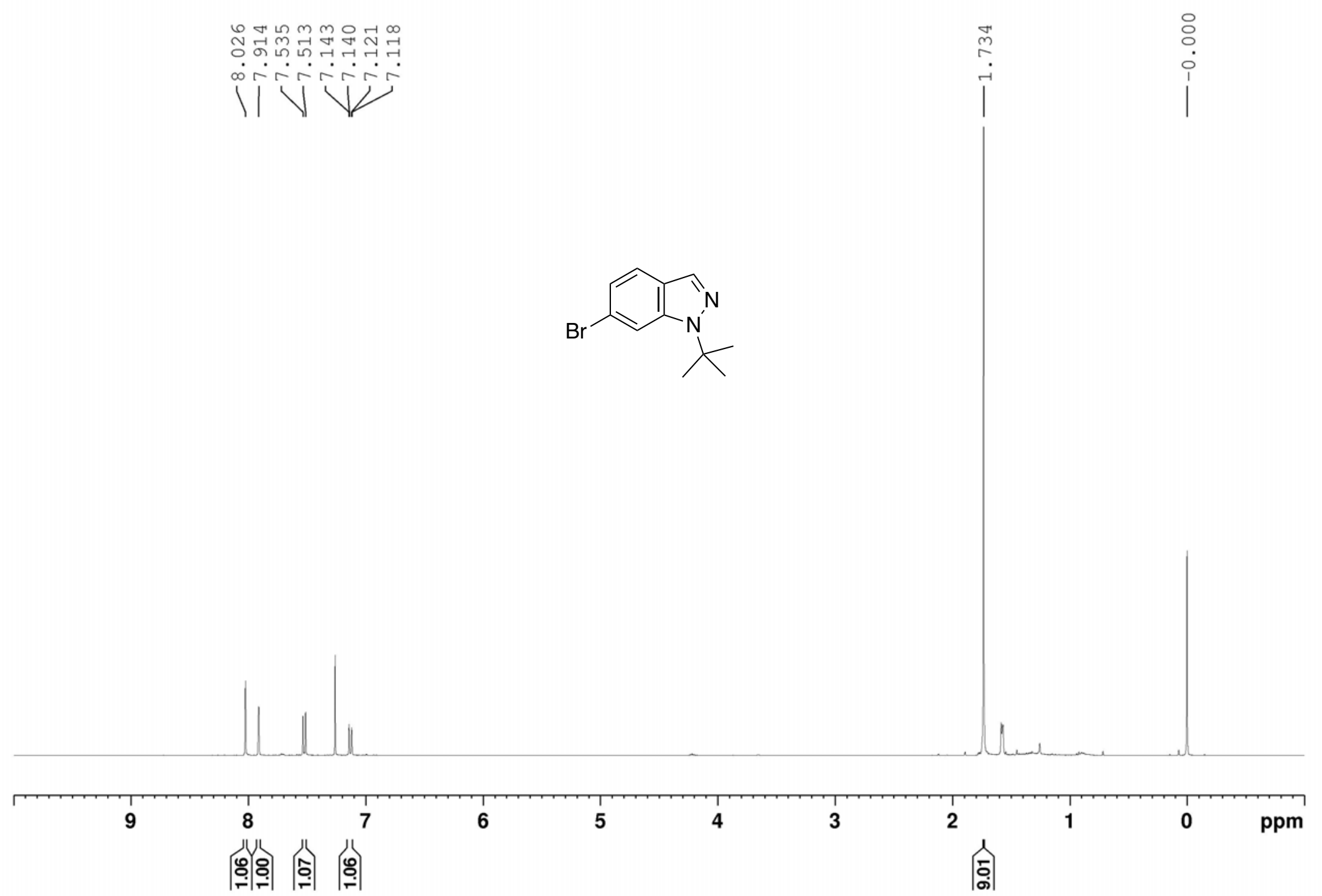

${ }^{1} \mathrm{H}$ NMR spectrum of $3 \mathbf{a a}\left(400 \mathrm{MHz}, \mathrm{CDCl}_{3}\right)$ 


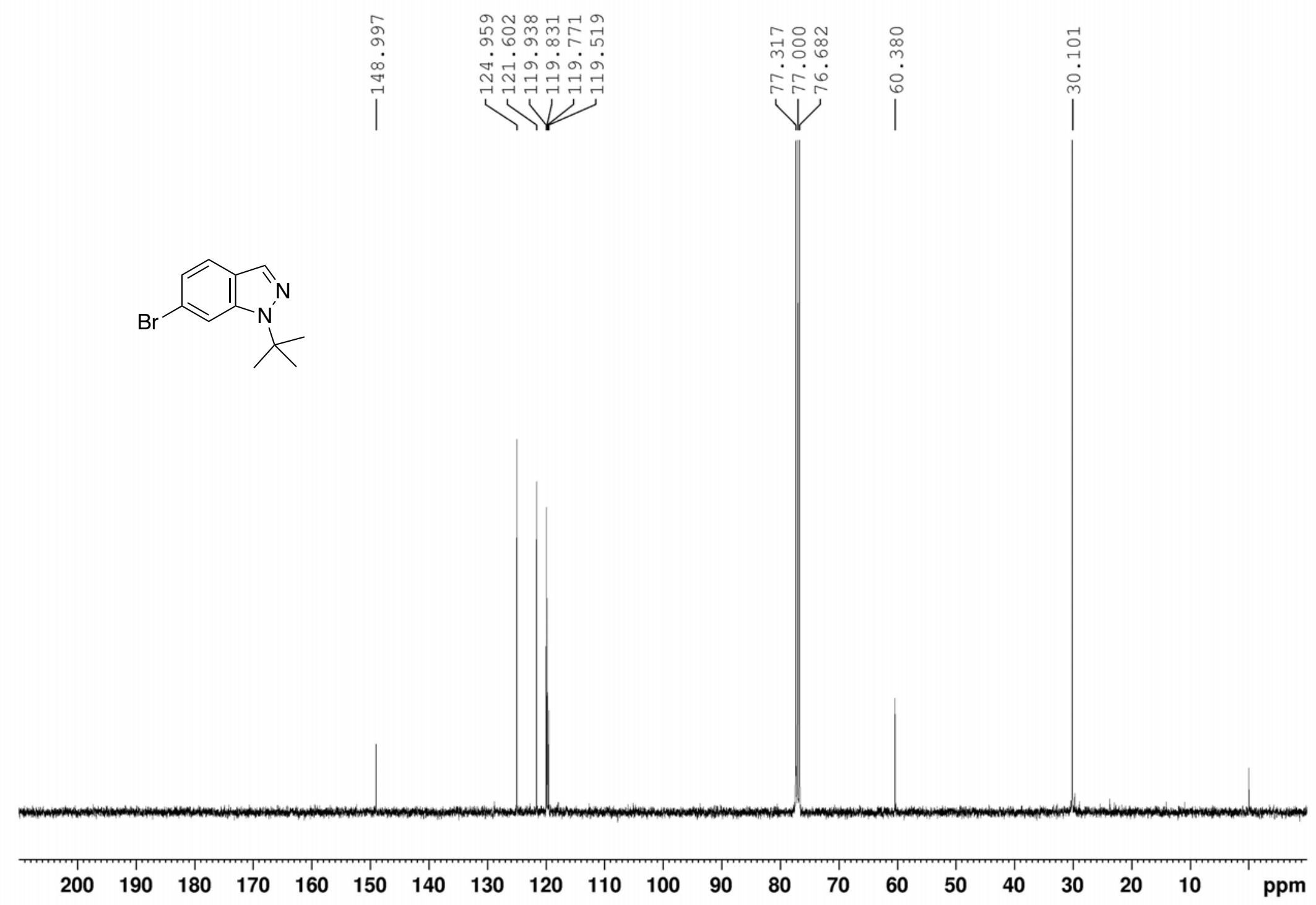

${ }^{13} \mathrm{C}$ NMR spectrum of $3 \mathbf{a a}\left(100.6 \mathrm{MHz}, \mathrm{CDCl}_{3}\right)$ 


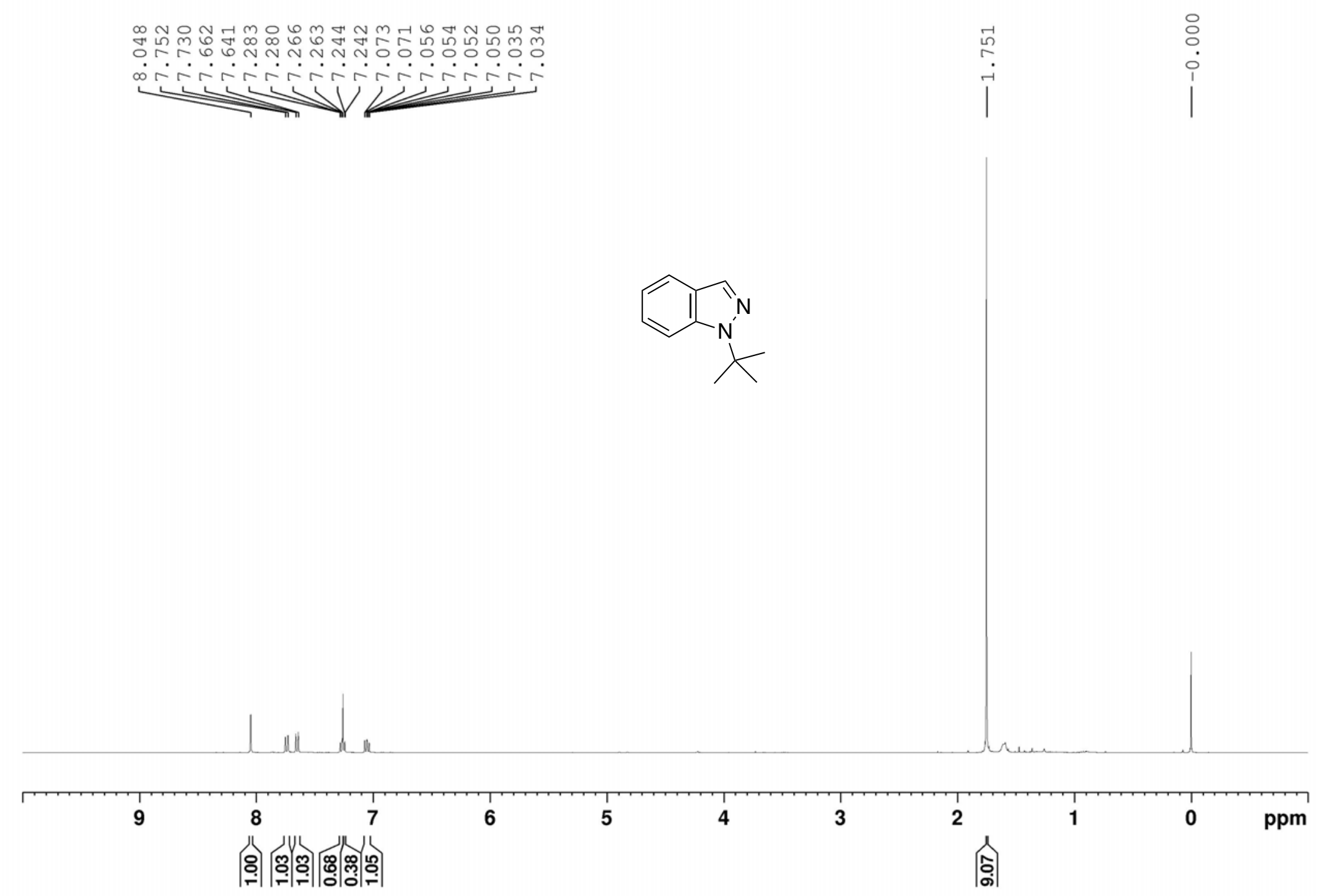

${ }^{1} \mathrm{H}$ NMR spectrum of $\mathbf{3 b a}\left(400 \mathrm{MHz}, \mathrm{CDCl}_{3}\right)$ 


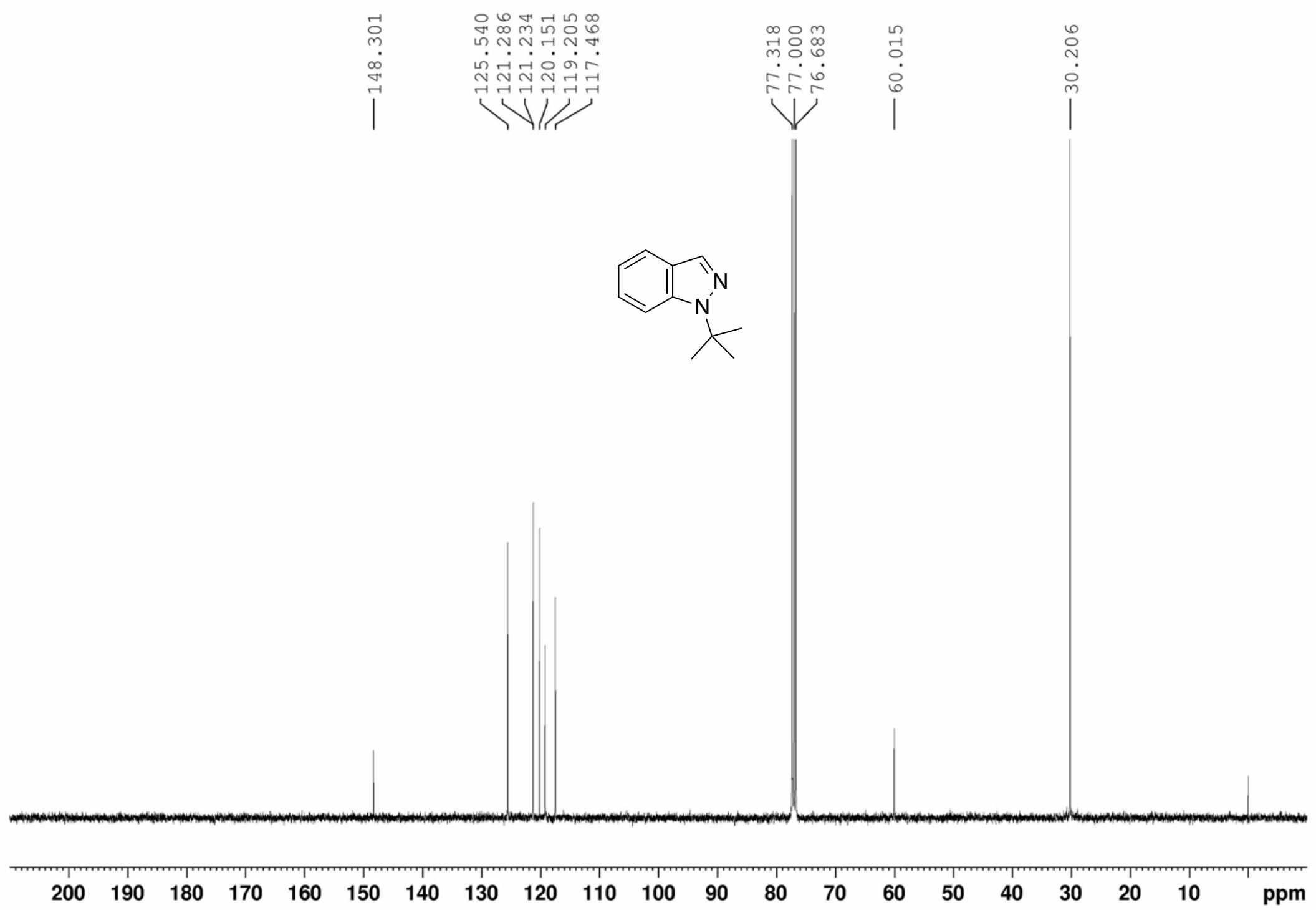

${ }^{13} \mathrm{C}$ NMR spectrum of $\mathbf{3 b a}\left(100.6 \mathrm{MHz}, \mathrm{CDCl}_{3}\right)$ 


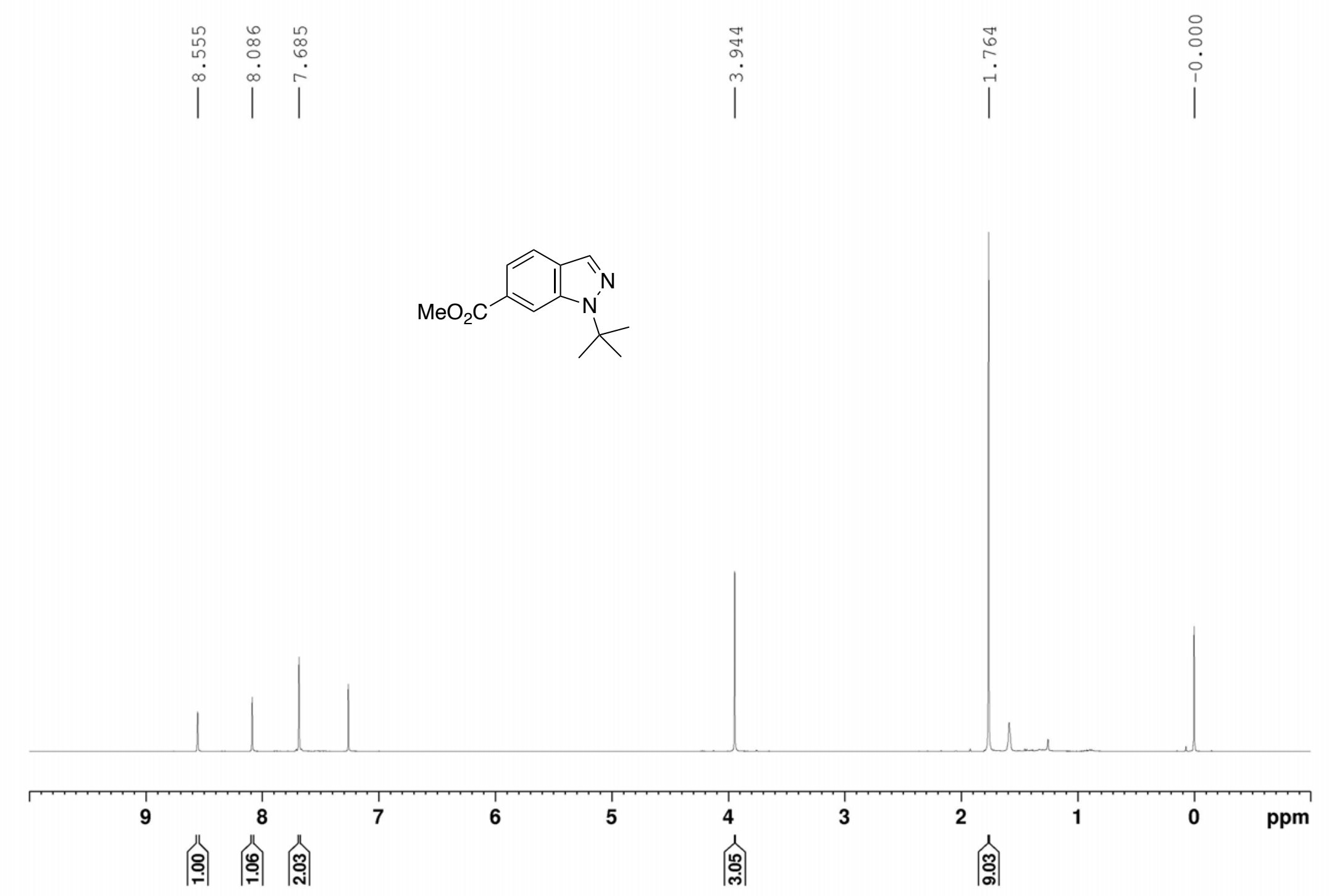

${ }^{1} \mathrm{H}$ NMR spectrum of 3ca $\left(400 \mathrm{MHz}, \mathrm{CDCl}_{3}\right)$ 


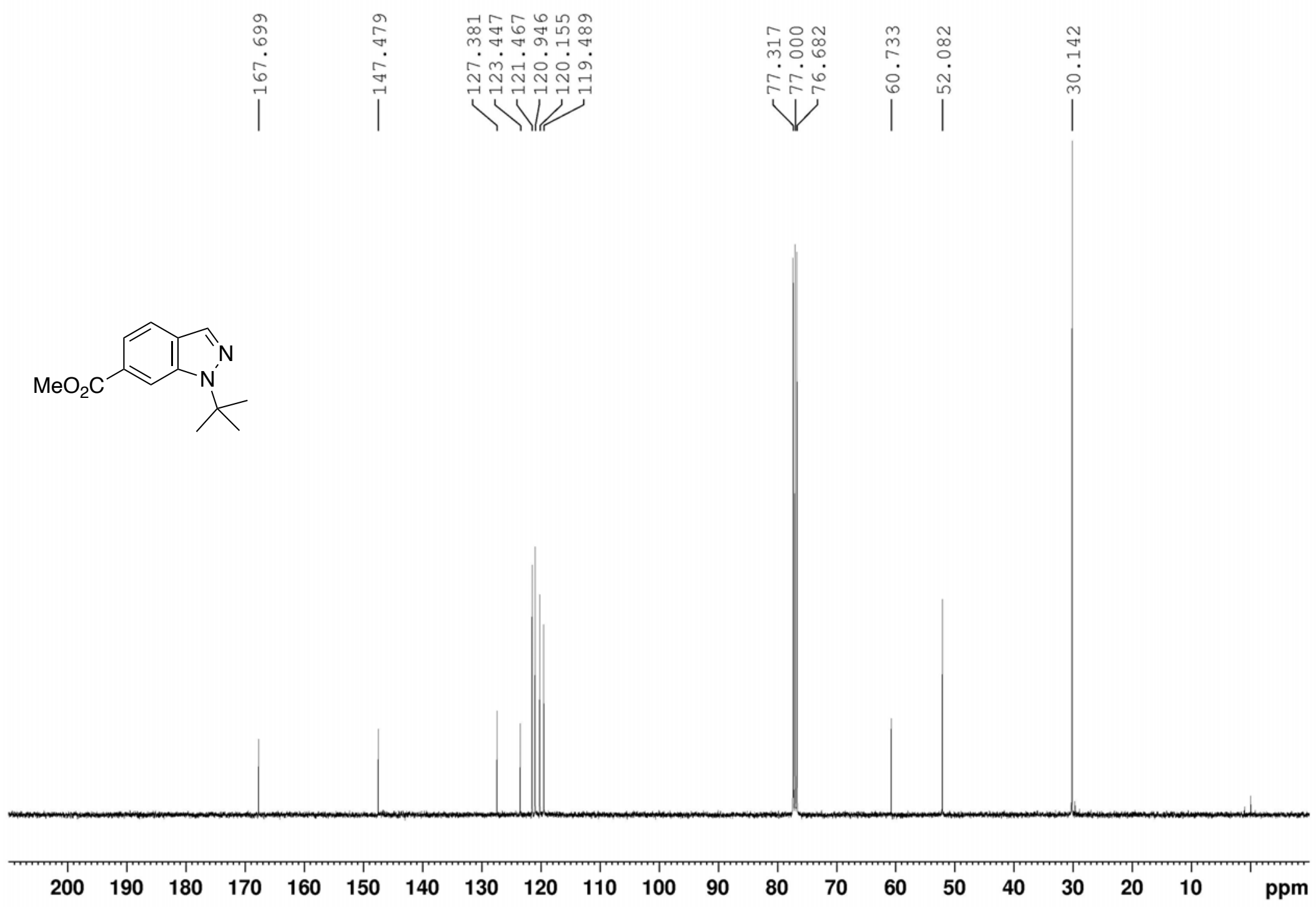

${ }^{13} \mathrm{C}$ NMR spectrum of $\mathbf{3 c a}\left(100.6 \mathrm{MHz}, \mathrm{CDCl}_{3}\right)$ 


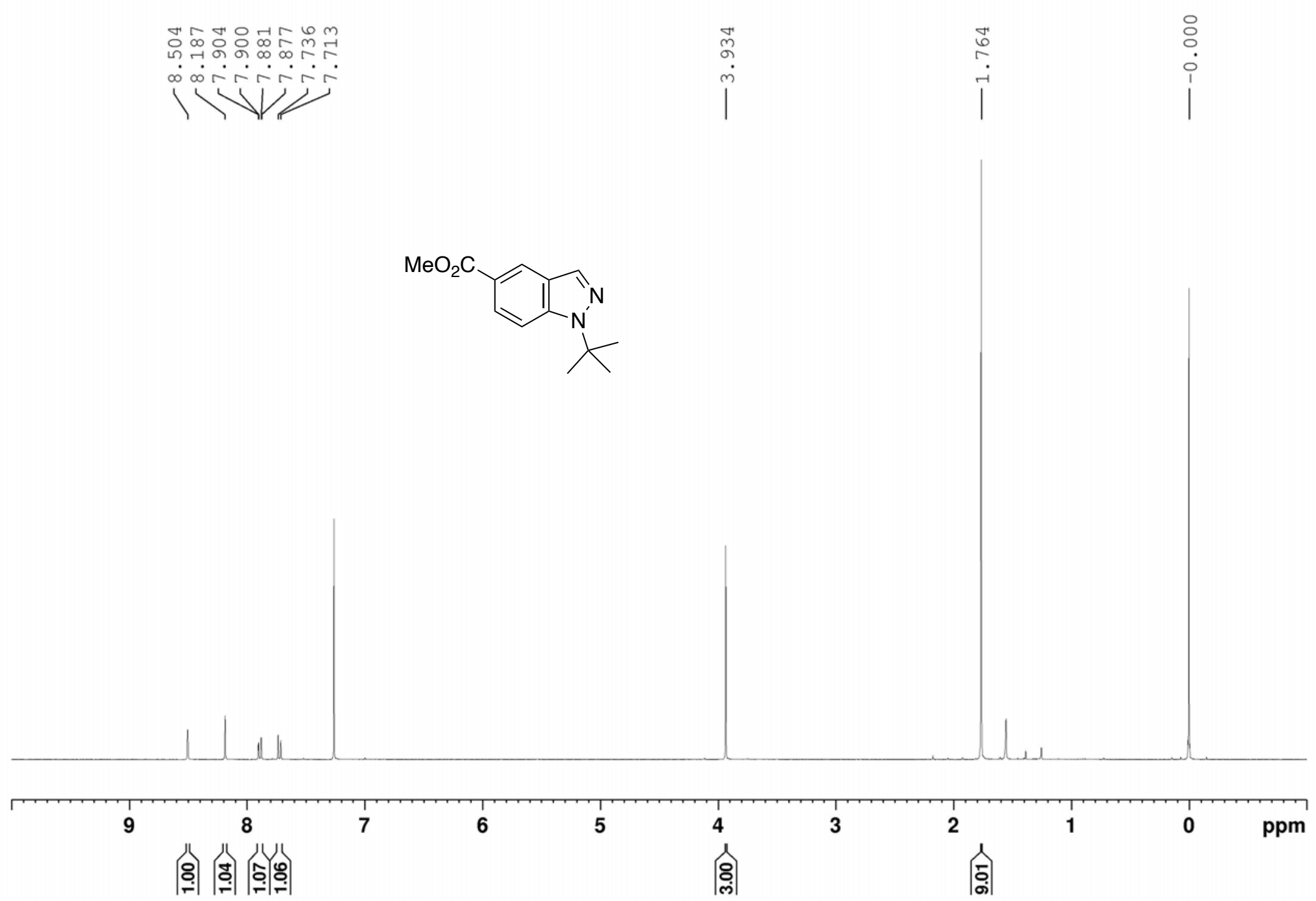

${ }^{1} \mathrm{H}$ NMR spectrum of $\mathbf{3 d a}\left(400 \mathrm{MHz}, \mathrm{CDCl}_{3}\right)$ 


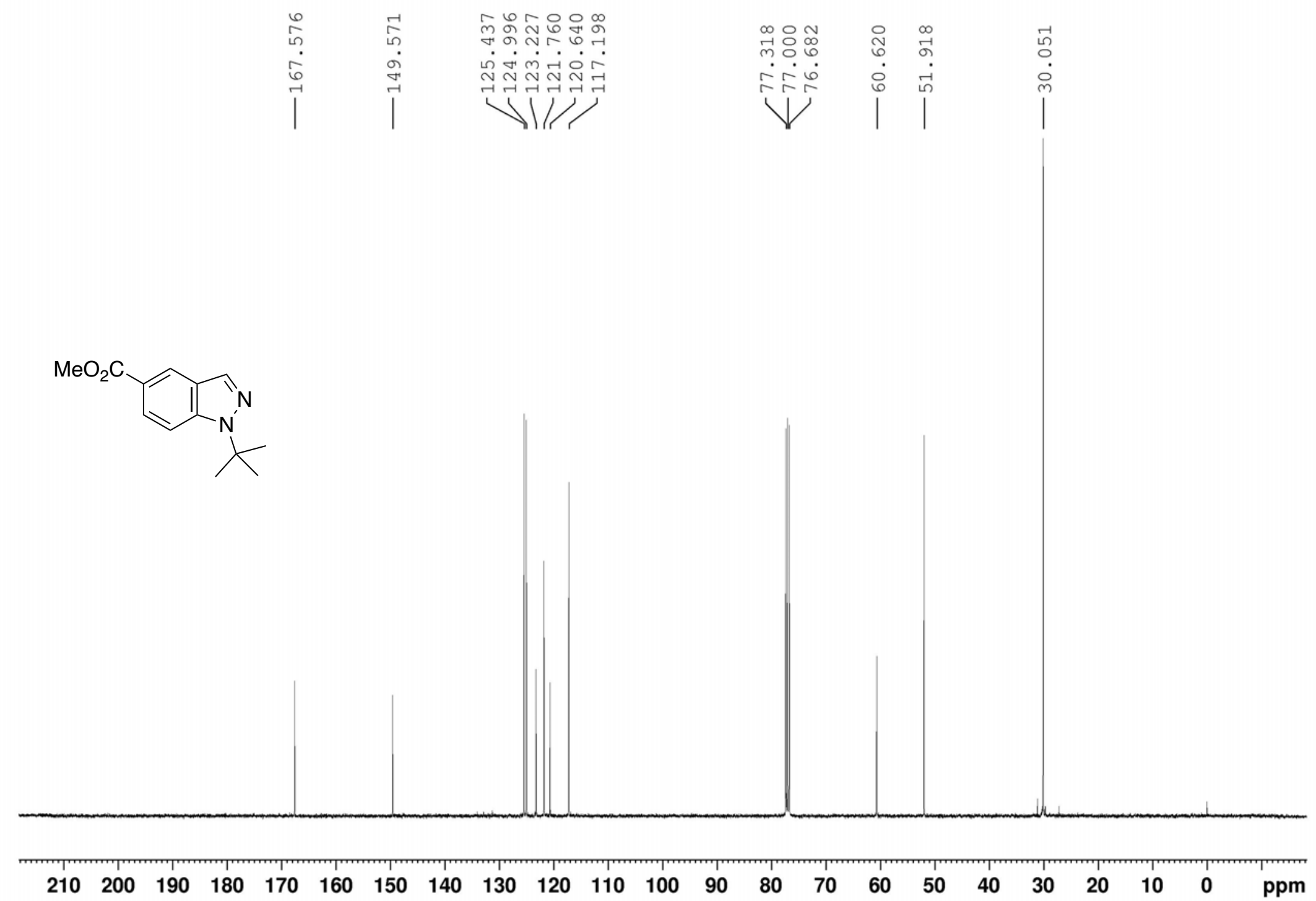

${ }^{13} \mathrm{C}$ NMR spectrum of $3 \mathbf{d a}\left(100.6 \mathrm{MHz}, \mathrm{CDCl}_{3}\right)$ 


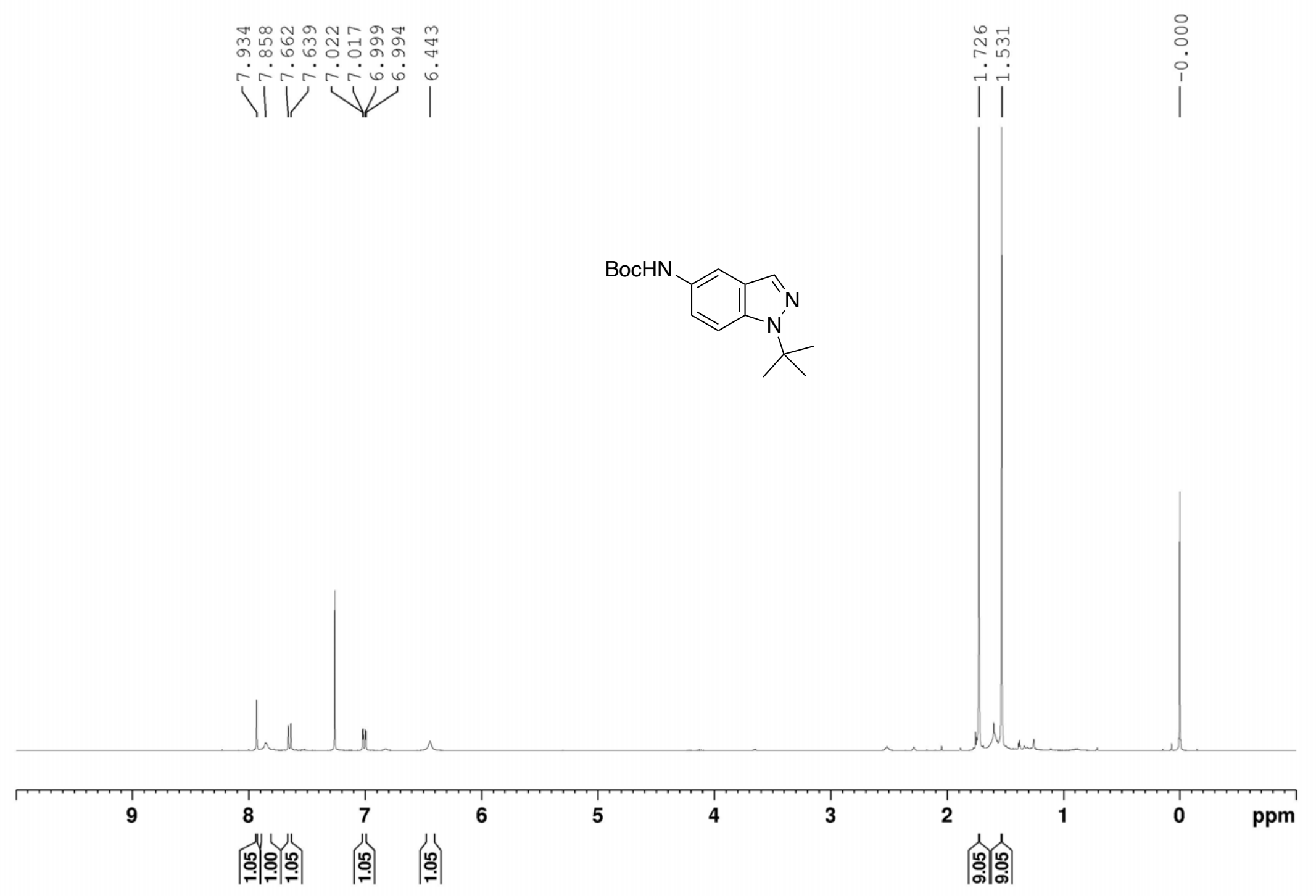

${ }^{1} \mathrm{H}$ NMR spectrum of 3ea $\left(400 \mathrm{MHz}, \mathrm{CDCl}_{3}\right)$ 


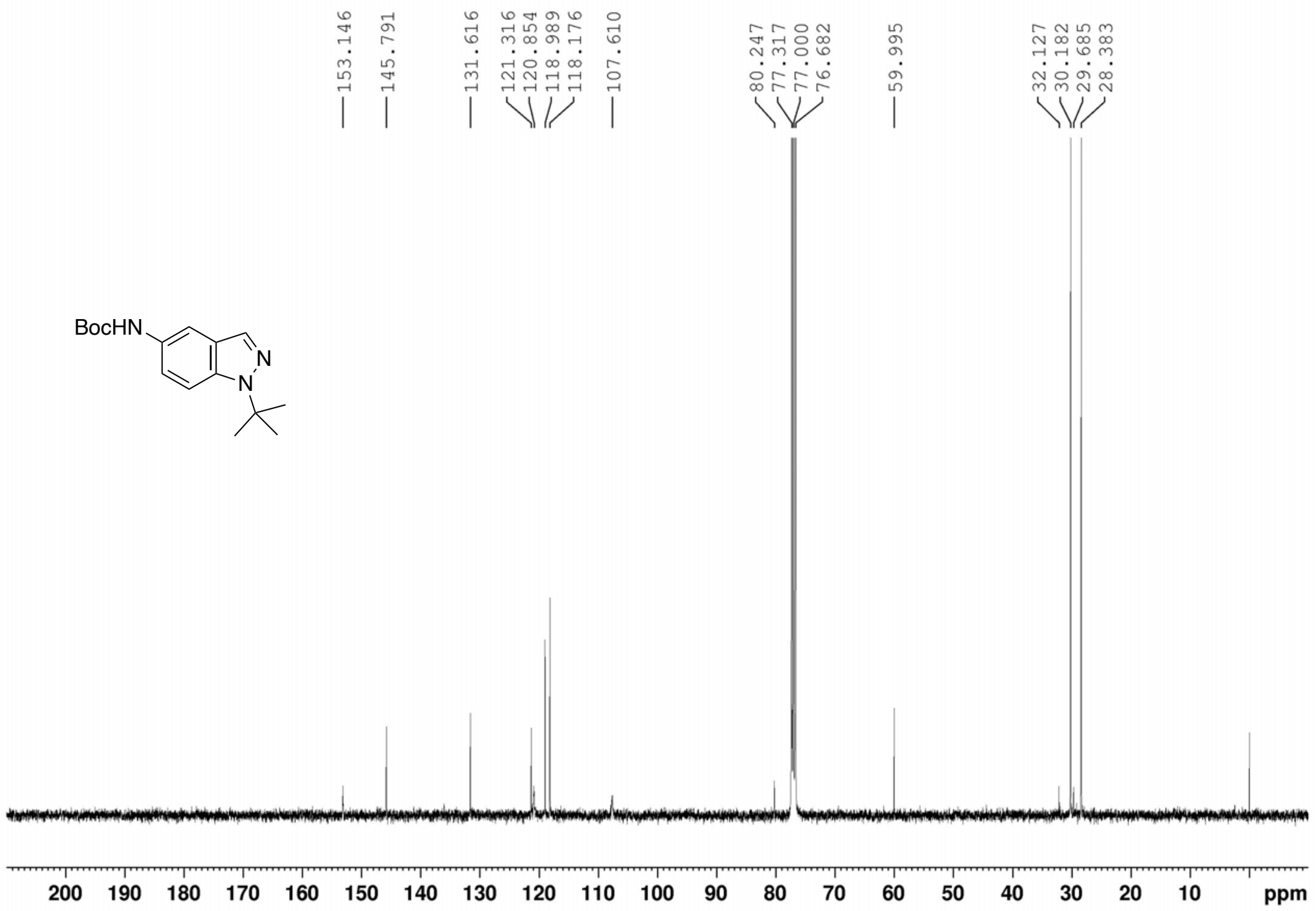

${ }^{13} \mathrm{C}$ NMR spectrum of $\mathbf{3 e a}\left(100.6 \mathrm{MHz}, \mathrm{CDCl}_{3}\right)$ 


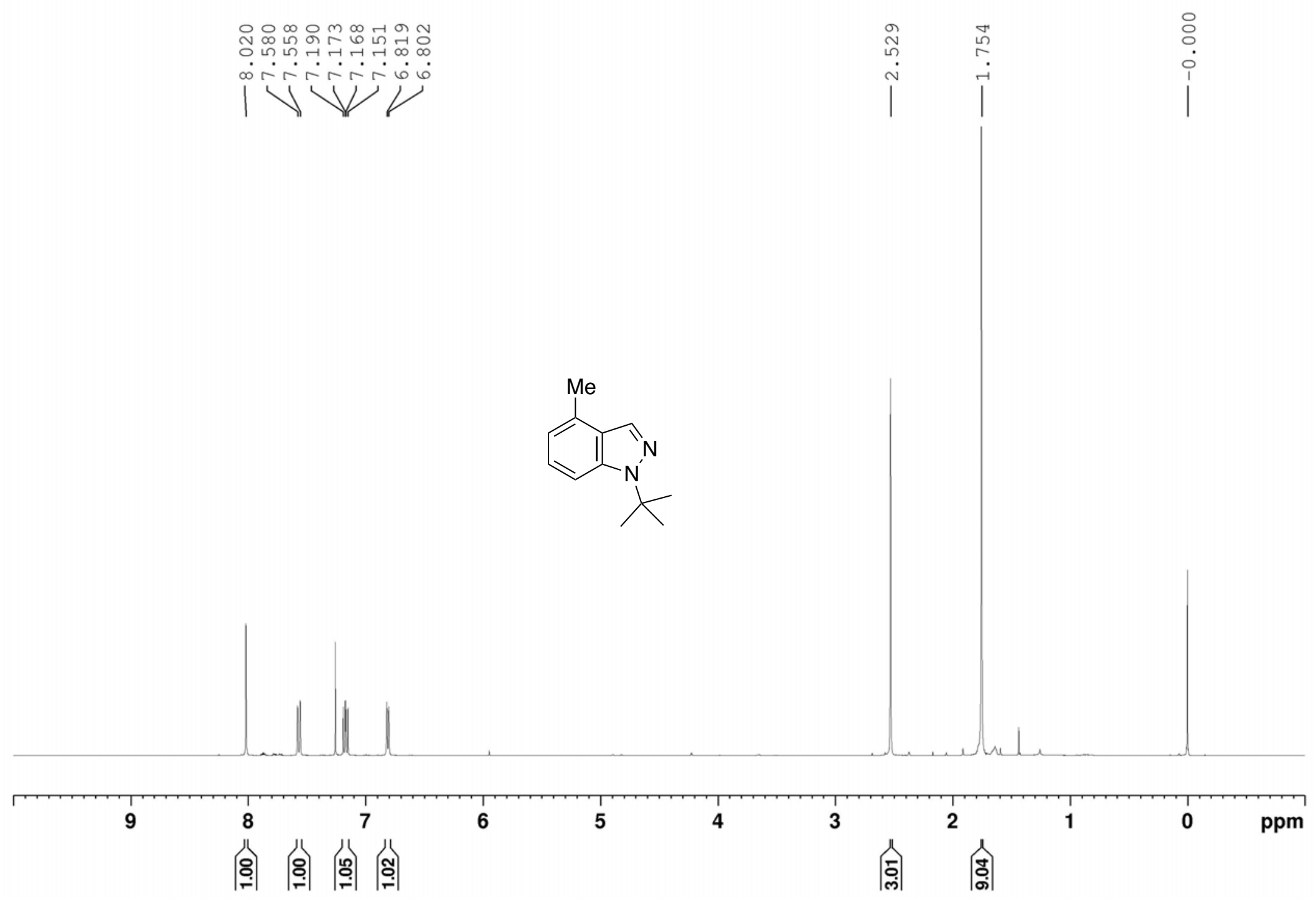

${ }^{1} \mathrm{H}$ NMR spectrum of $\mathbf{3 f a}\left(400 \mathrm{MHz}, \mathrm{CDCl}_{3}\right)$ 


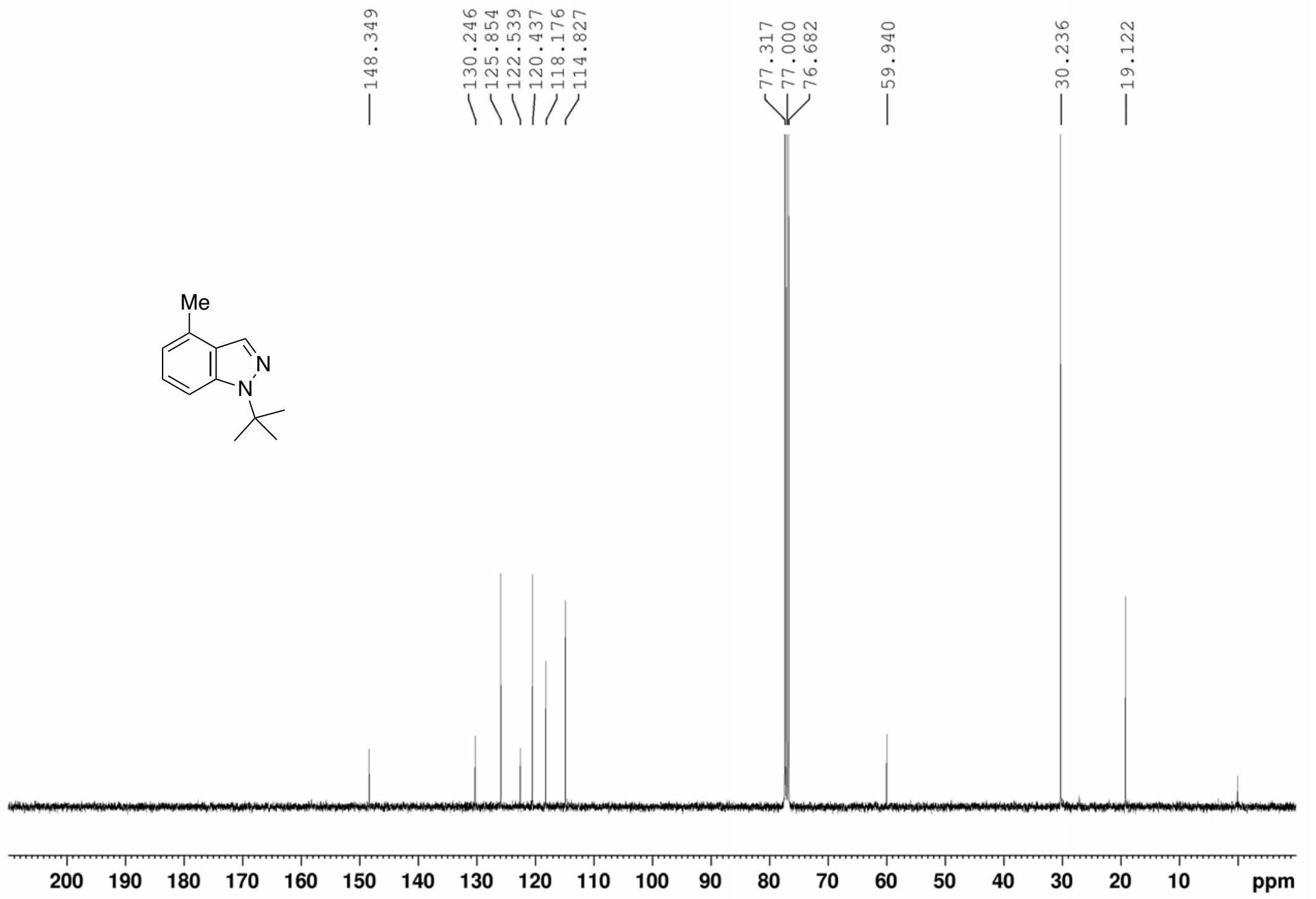

${ }^{13} \mathrm{C}$ NMR spectrum of $\mathbf{3 f a}\left(100.6 \mathrm{MHz}, \mathrm{CDCl}_{3}\right)$ 


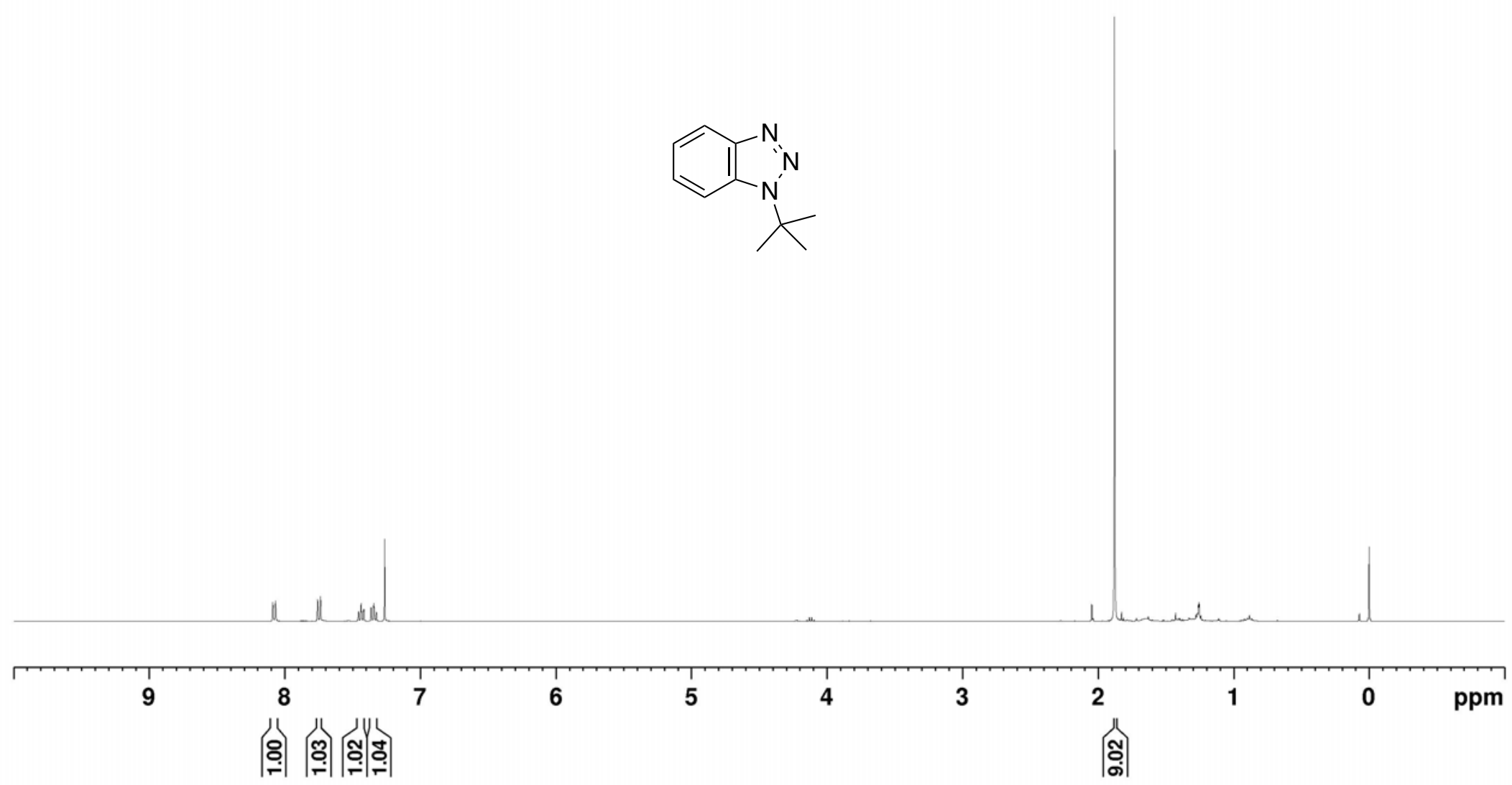

${ }^{1} \mathrm{H}$ NMR spectrum of $\mathbf{3 g a}\left(400 \mathrm{MHz}, \mathrm{CDCl}_{3}\right)$ 


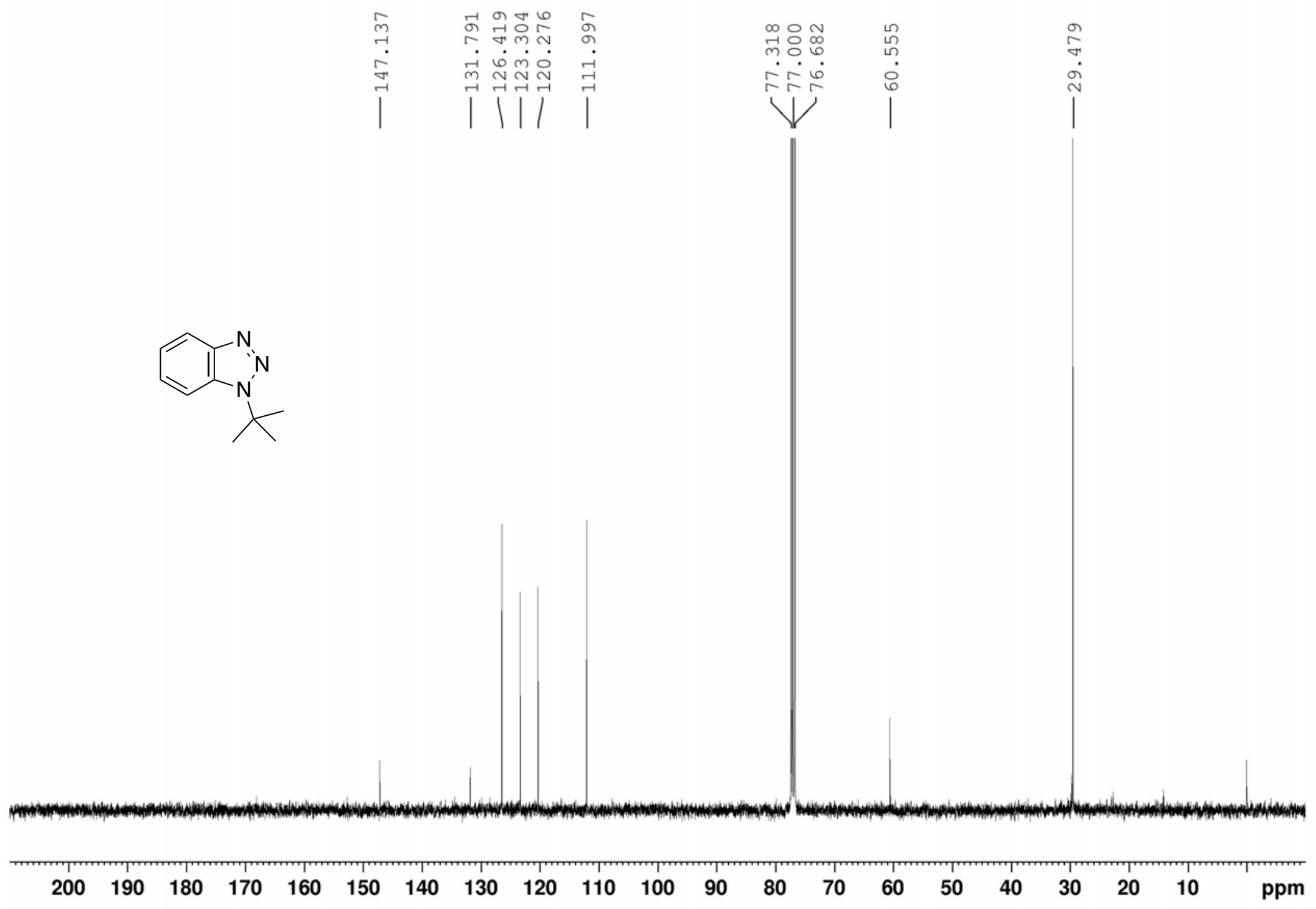

${ }^{13} \mathrm{C}$ NMR spectrum of 3 ga $\left(100.6 \mathrm{MHz}, \mathrm{CDCl}_{3}\right)$ 


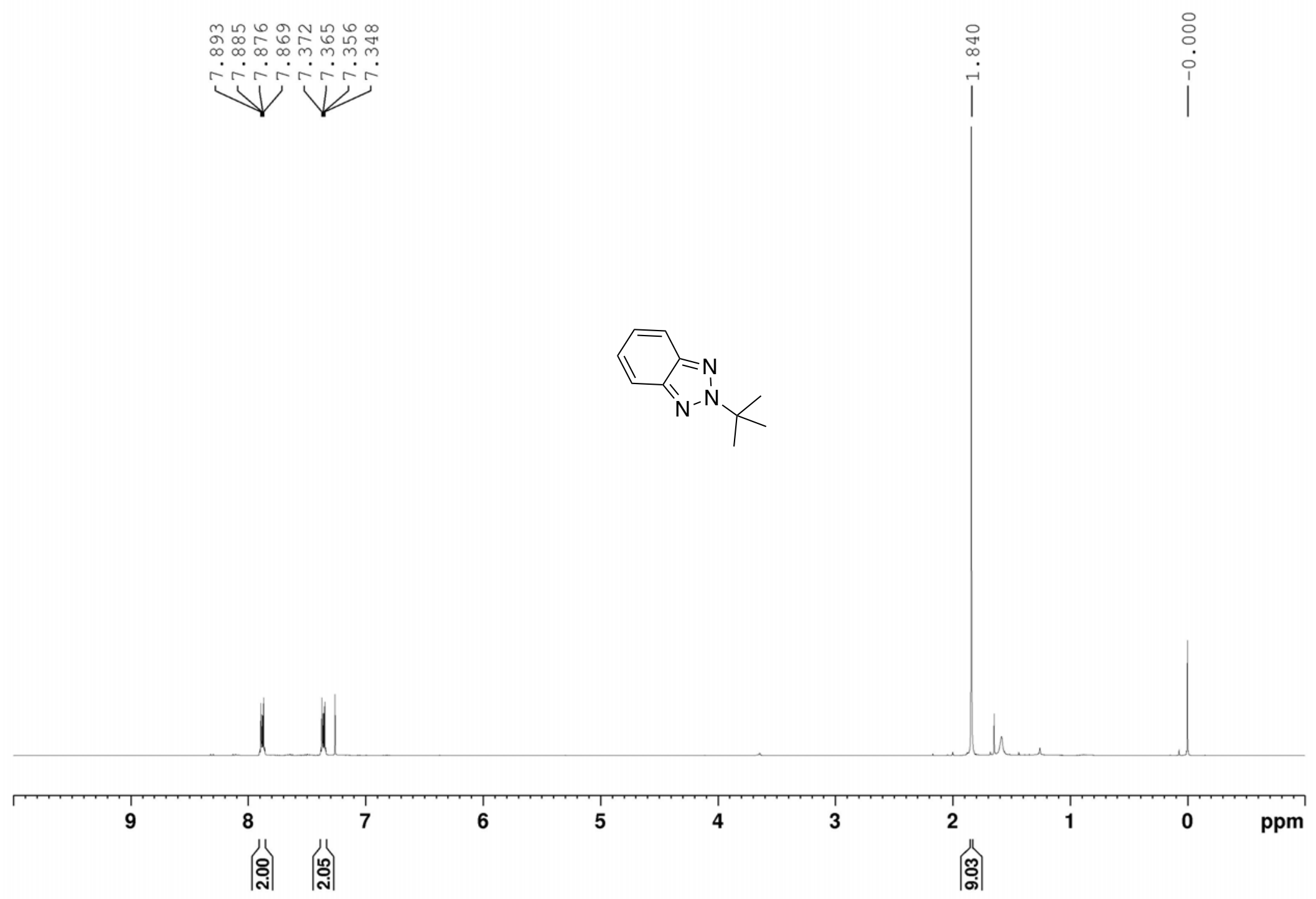

${ }^{1} \mathrm{H}$ NMR spectrum of $\mathbf{3 g a}{ }^{\prime}\left(400 \mathrm{MHz}, \mathrm{CDCl}_{3}\right)$ 


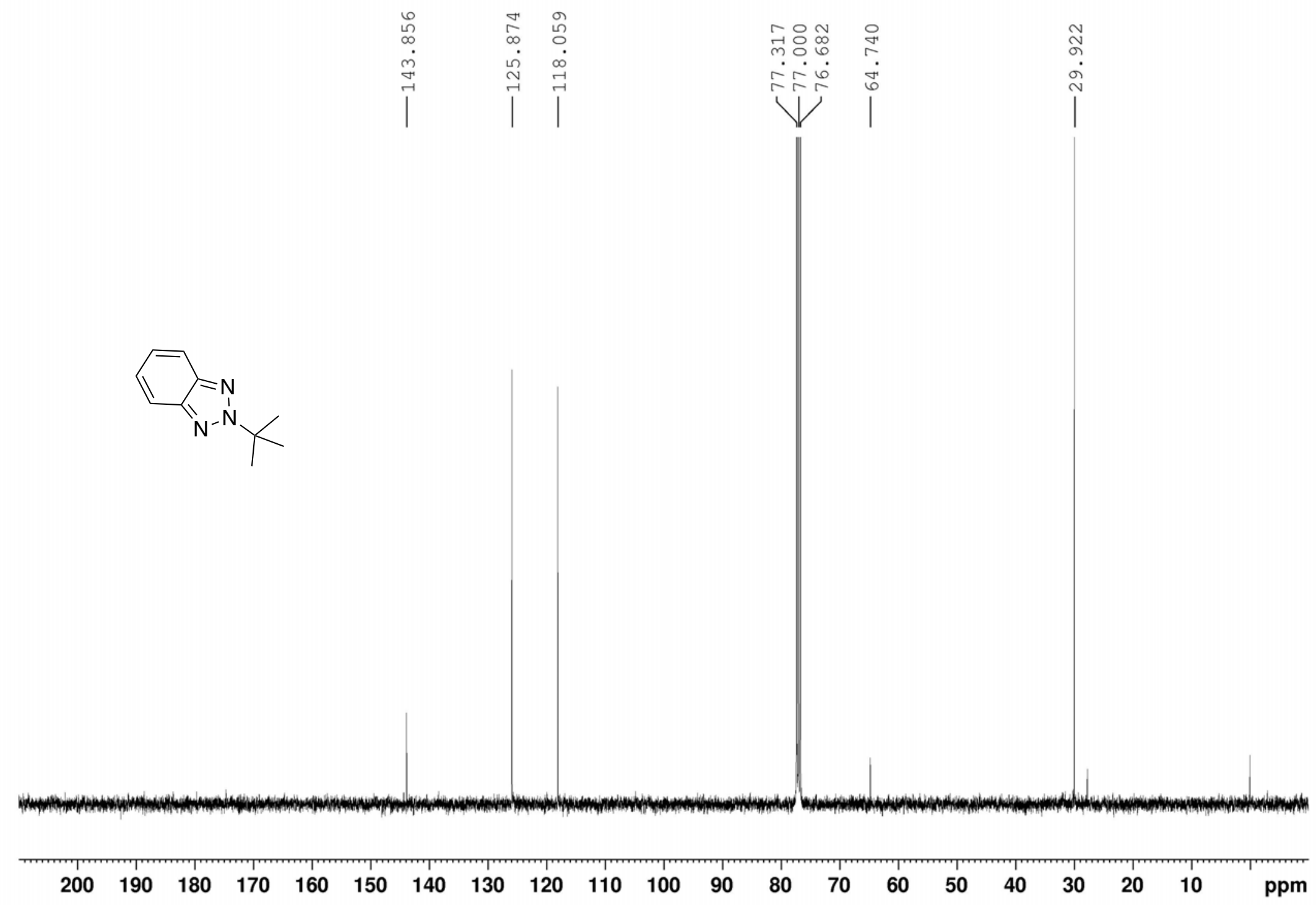

${ }^{13} \mathrm{C}$ NMR spectrum of 3ga' $\left(100.6 \mathrm{MHz}, \mathrm{CDCl}_{3}\right)$ 


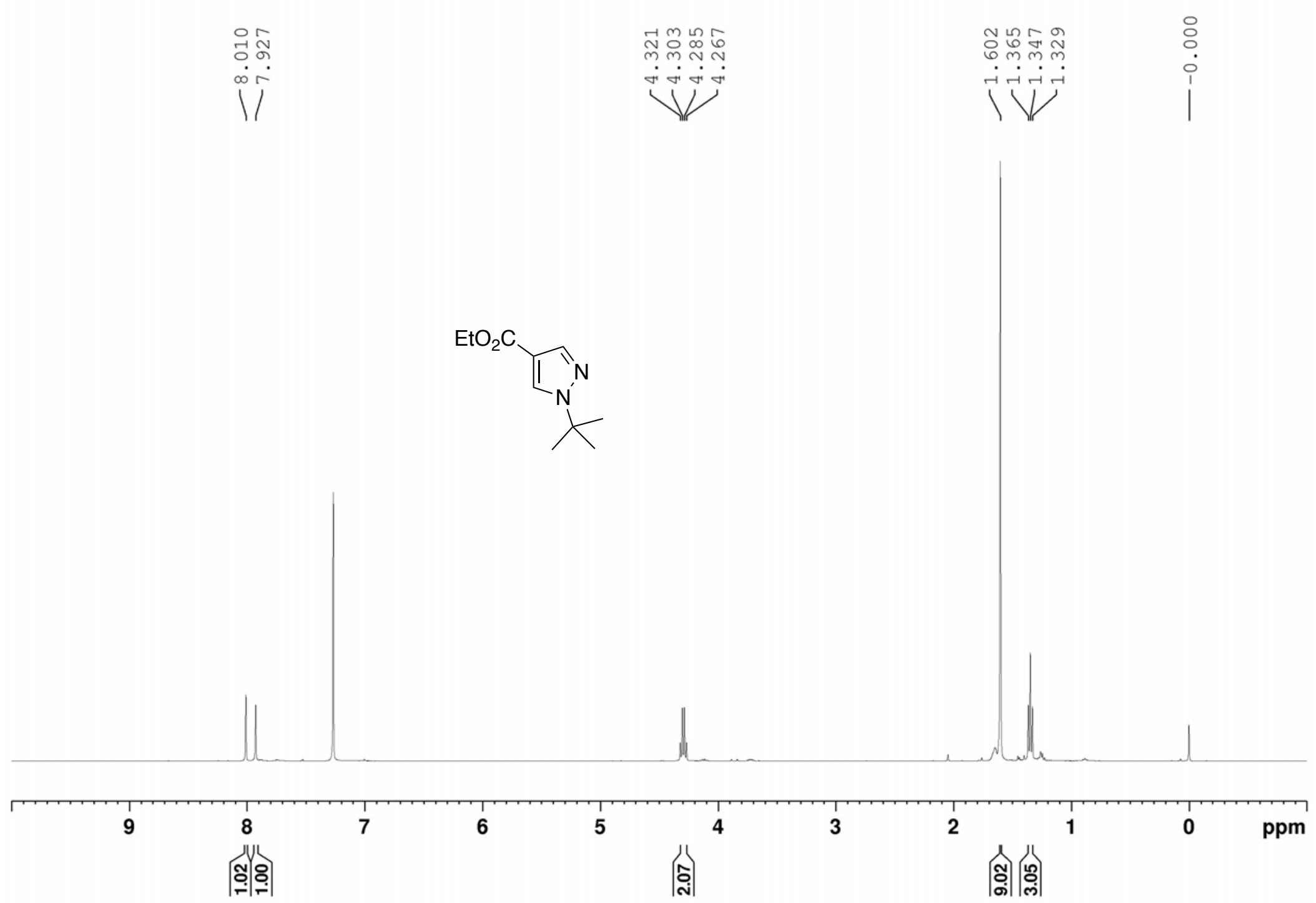

${ }^{1} \mathrm{H}$ NMR spectrum of $\mathbf{3 h a}\left(400 \mathrm{MHz}, \mathrm{CDCl}_{3}\right)$ 


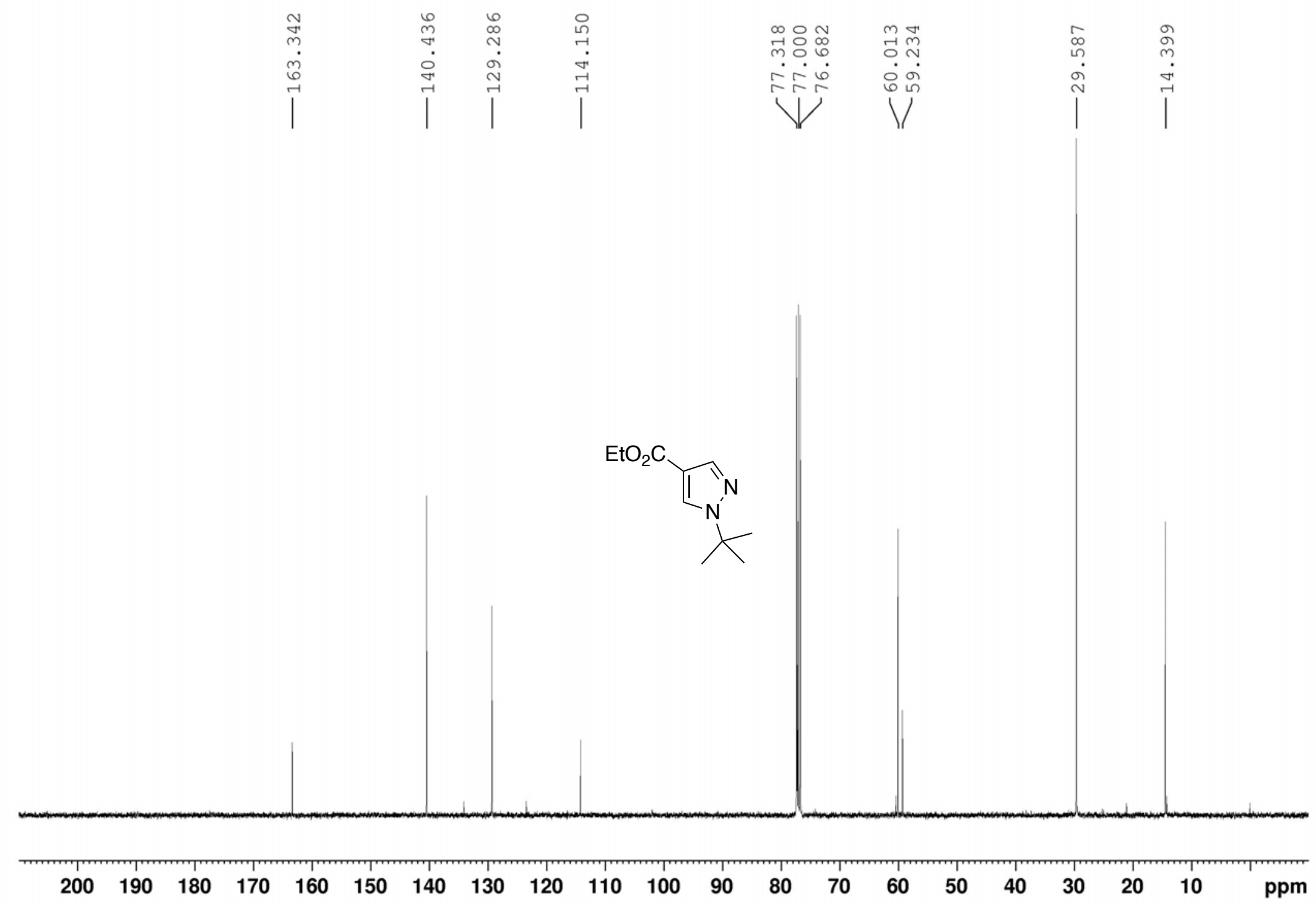

${ }^{13} \mathrm{C}$ NMR spectrum of $\mathbf{3 h a}\left(100.6 \mathrm{MHz}, \mathrm{CDCl}_{3}\right)$ 


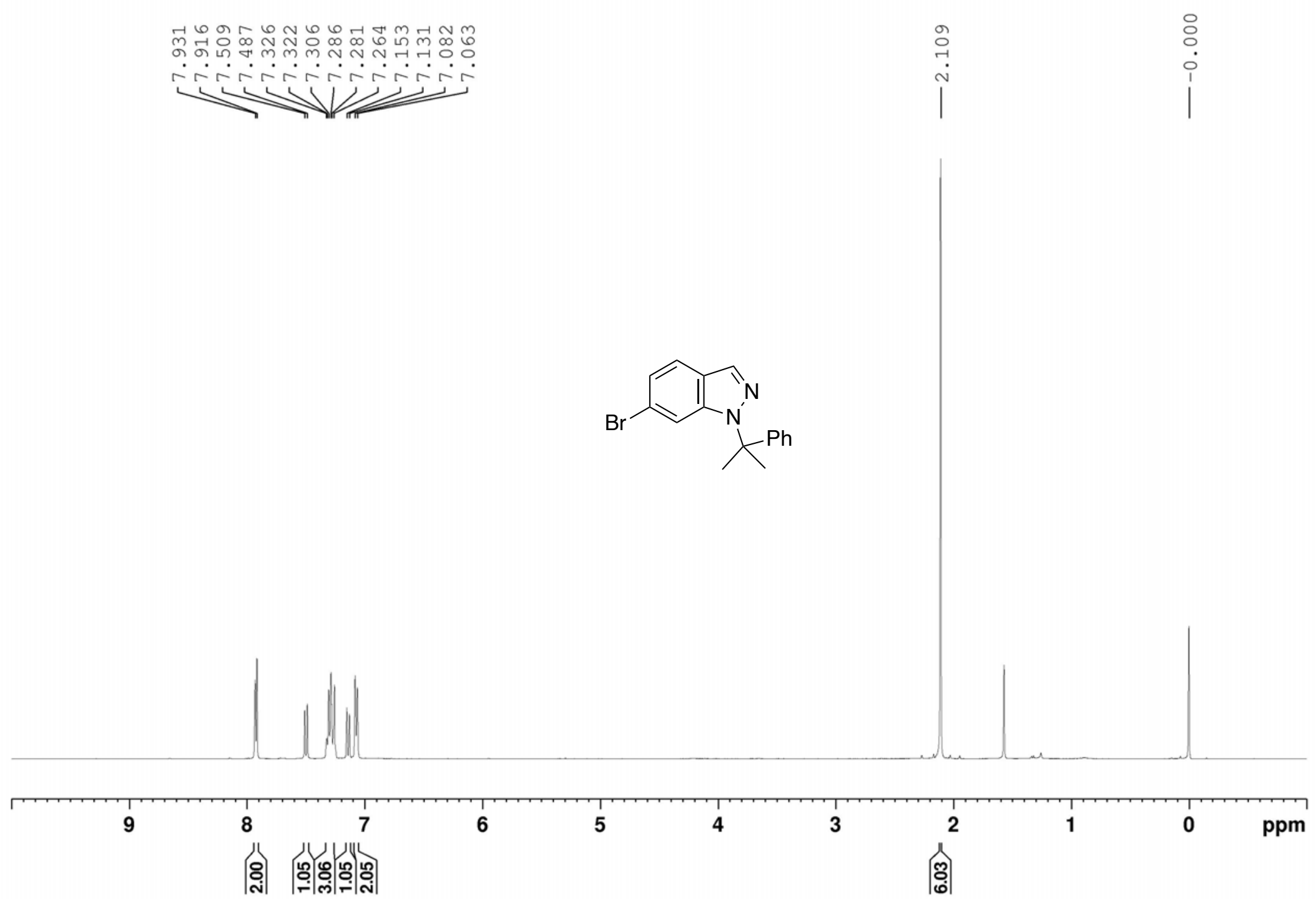

${ }^{1} \mathrm{H}$ NMR spectrum of $\mathbf{3 a b}\left(400 \mathrm{MHz}, \mathrm{CDCl}_{3}\right)$ 


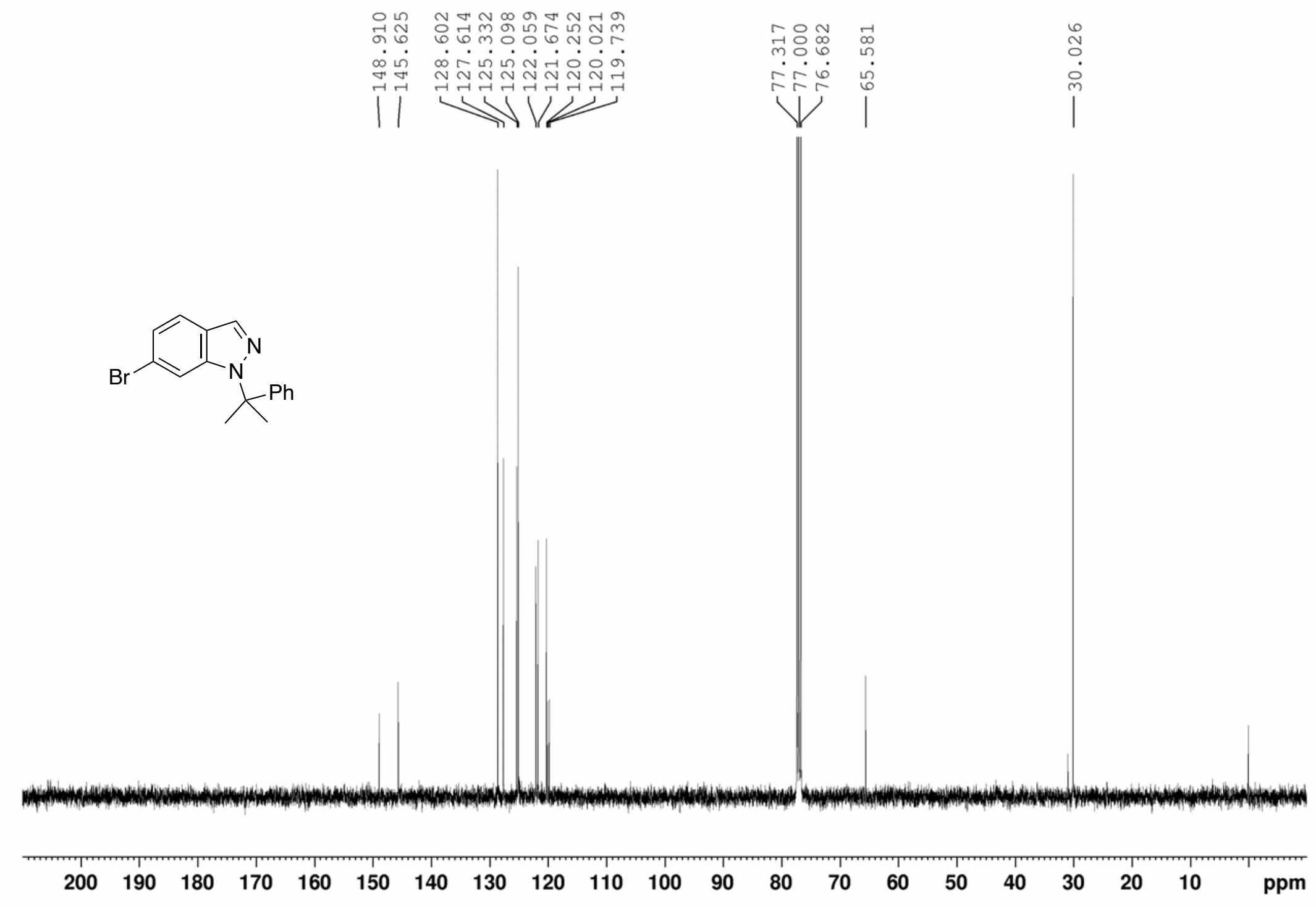

${ }^{13} \mathrm{C}$ NMR spectrum of $\mathbf{3 a b}\left(100.6 \mathrm{MHz}, \mathrm{CDCl}_{3}\right)$ 


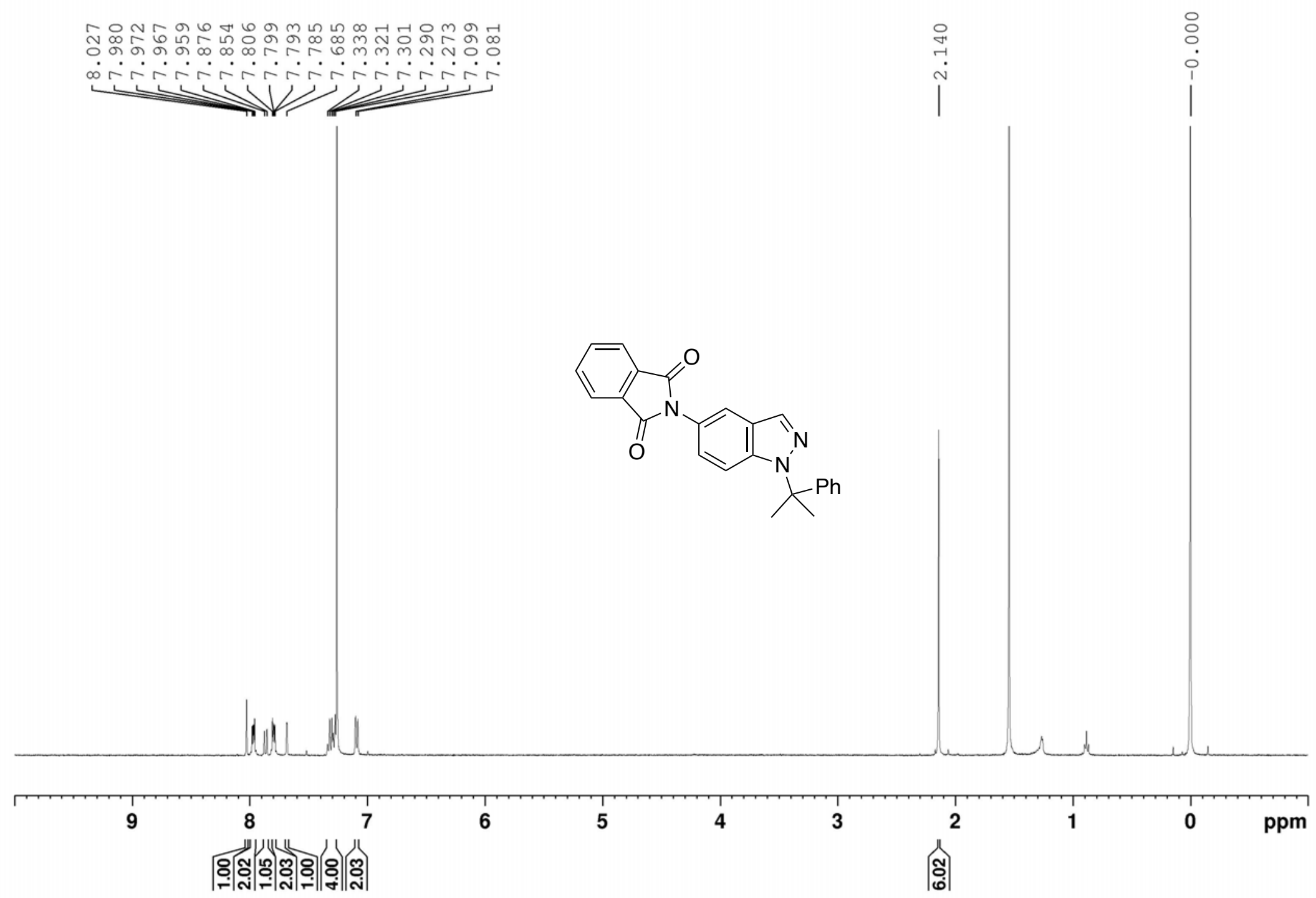

${ }^{1} \mathrm{H}$ NMR spectrum of $3 \mathbf{i b}\left(400 \mathrm{MHz}, \mathrm{CDCl}_{3}\right)$ 


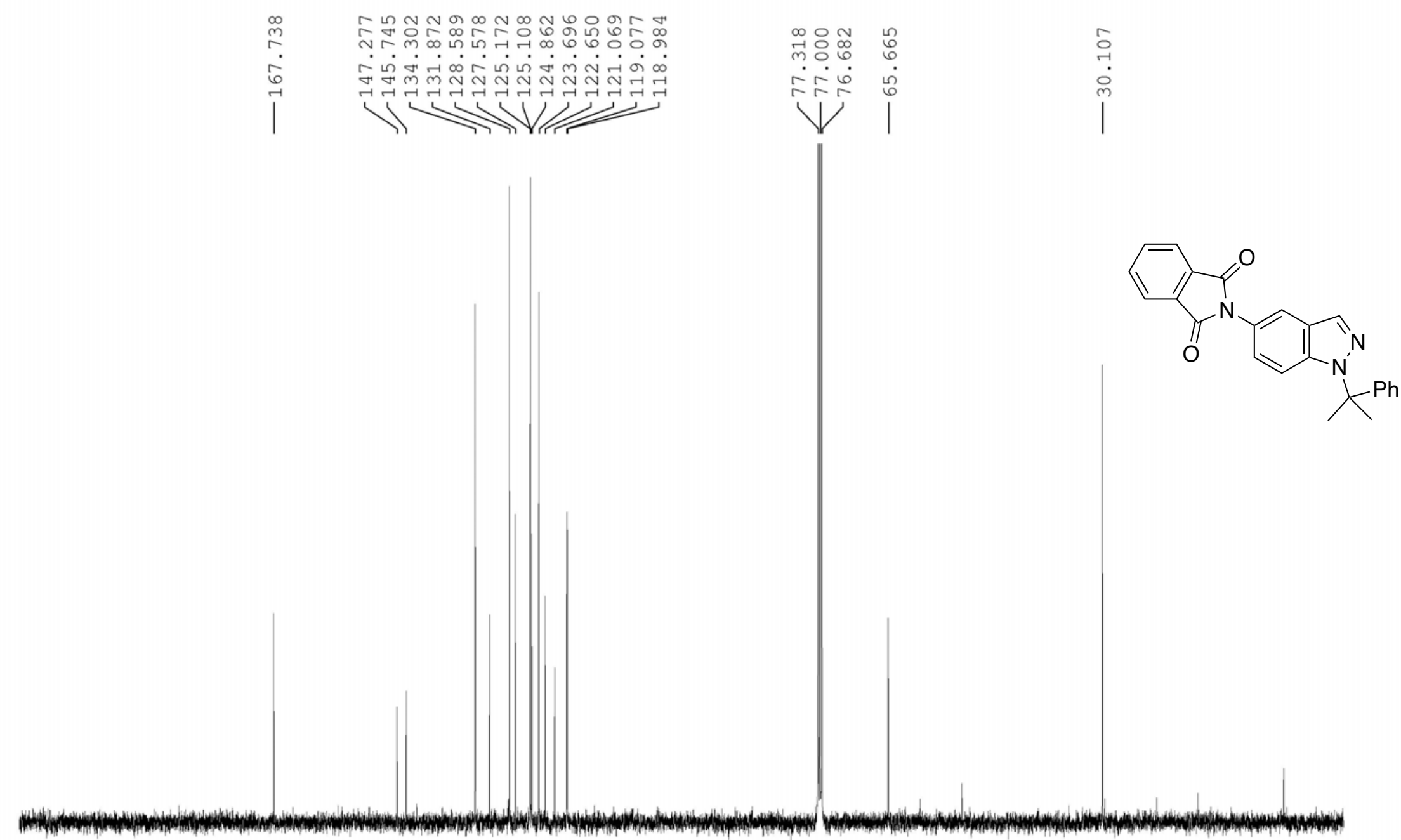

$\begin{array}{lllllllllllllllllllll}200 & 190 & 180 & 170 & 160 & 150 & 140 & 130 & 120 & 110 & 100 & 90 & 80 & 70 & 60 & 50 & 40 & 30 & 20 & 10 & \text { ppm }\end{array}$

${ }^{13} \mathrm{C}$ NMR spectrum of $3 \mathbf{i b}\left(100.6 \mathrm{MHz}, \mathrm{CDCl}_{3}\right)$ 


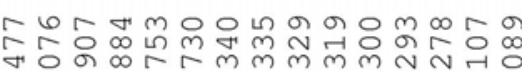

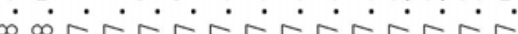

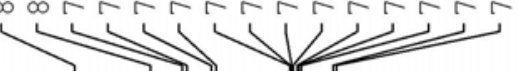

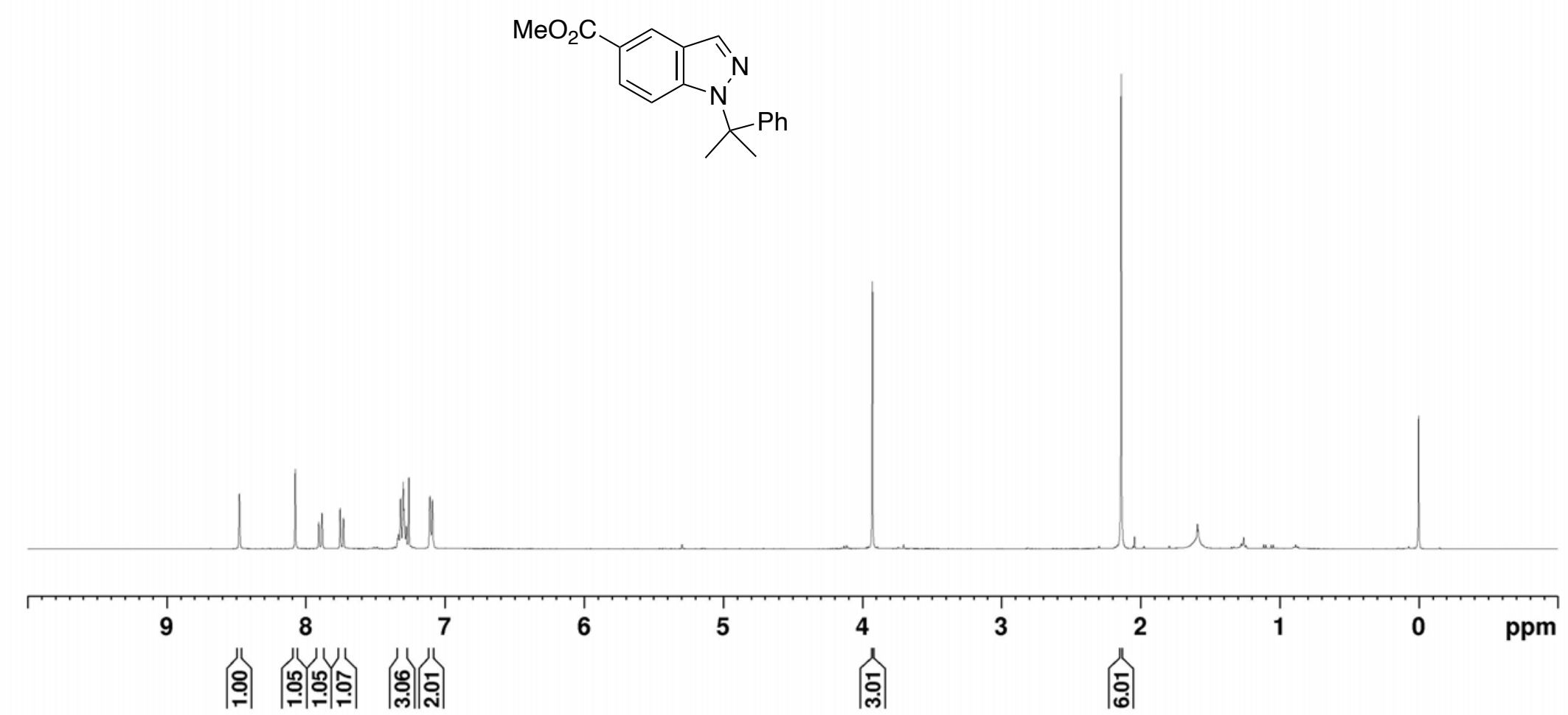

${ }^{1} \mathrm{H}$ NMR spectrum of $\mathbf{3 d b}\left(400 \mathrm{MHz}, \mathrm{CDCl}_{3}\right)$ 


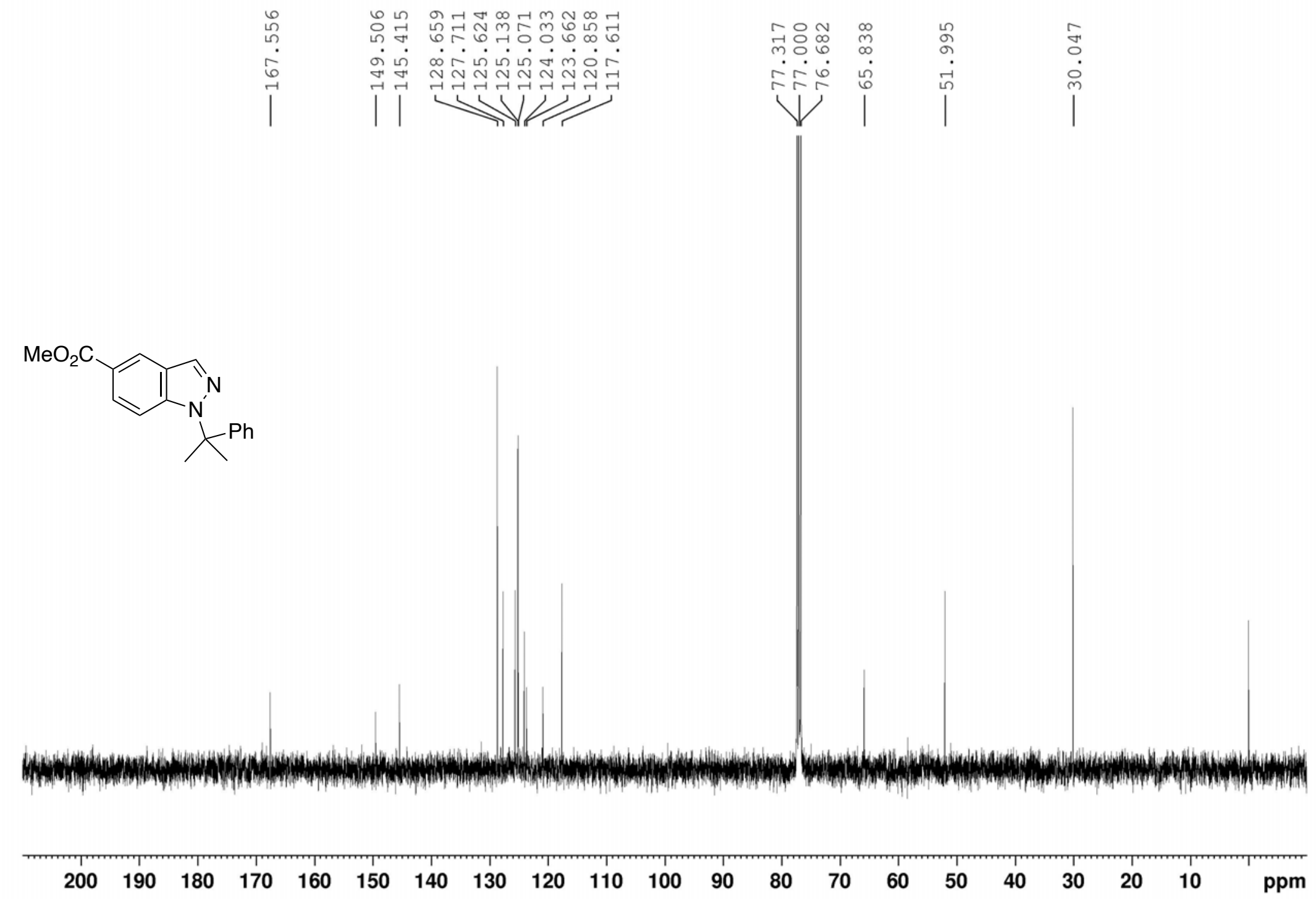

${ }^{13} \mathrm{C}$ NMR spectrum of $\mathbf{3 d b}\left(100.6 \mathrm{MHz}, \mathrm{CDCl}_{3}\right)$ 

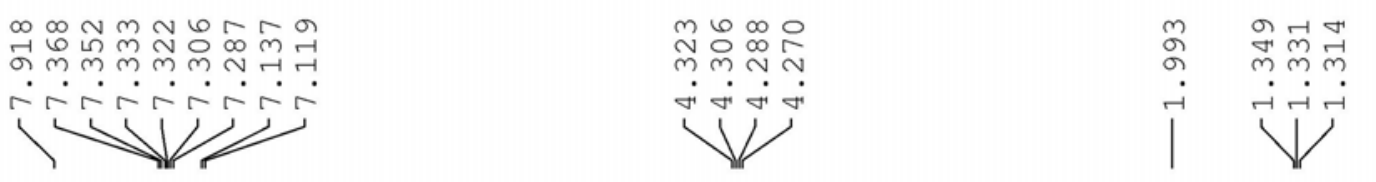

$$
\begin{aligned}
& \circ \\
& 0 \\
& 1 \\
& 1
\end{aligned}
$$

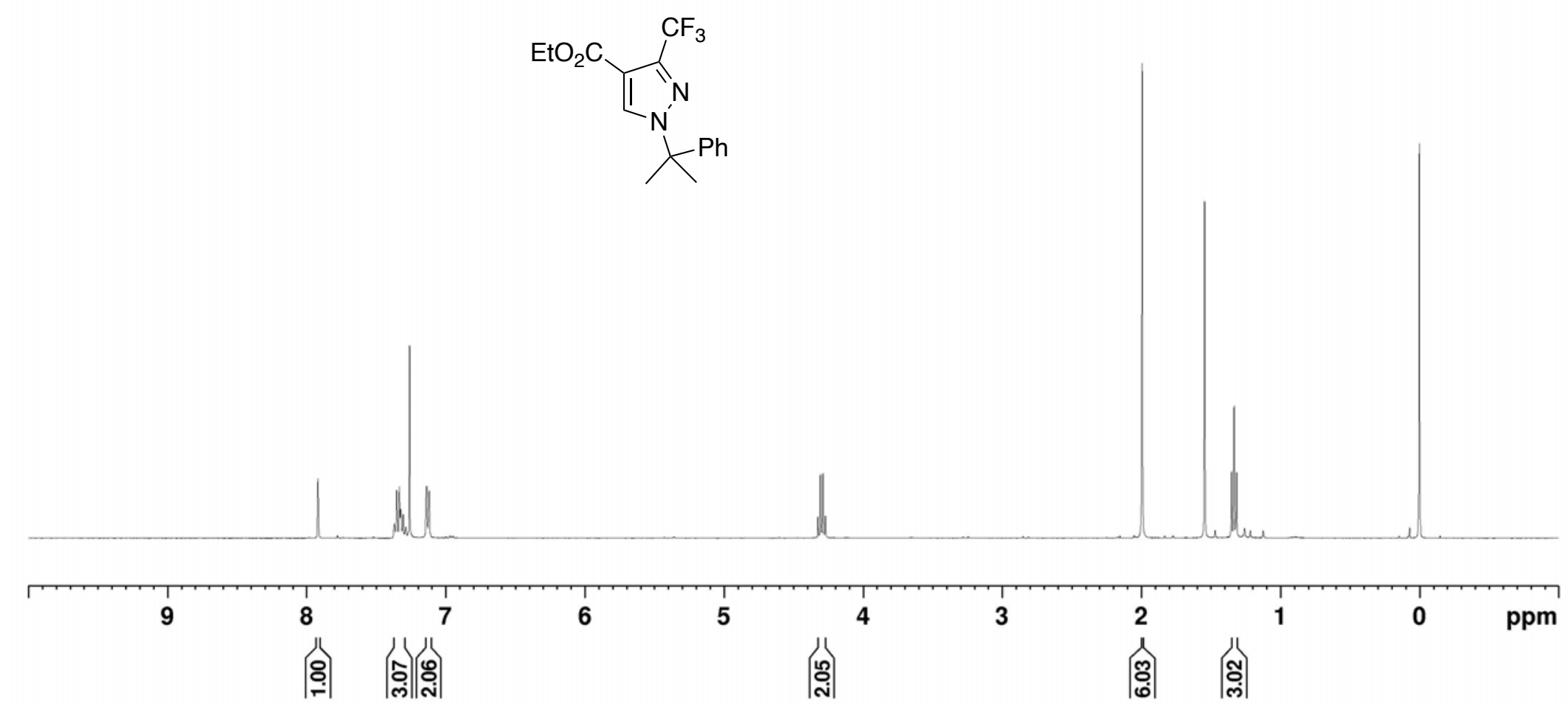

${ }^{1} \mathrm{H}$ NMR spectrum of $\mathbf{3 j b}\left(400 \mathrm{MHz}, \mathrm{CDCl}_{3}\right)$ 


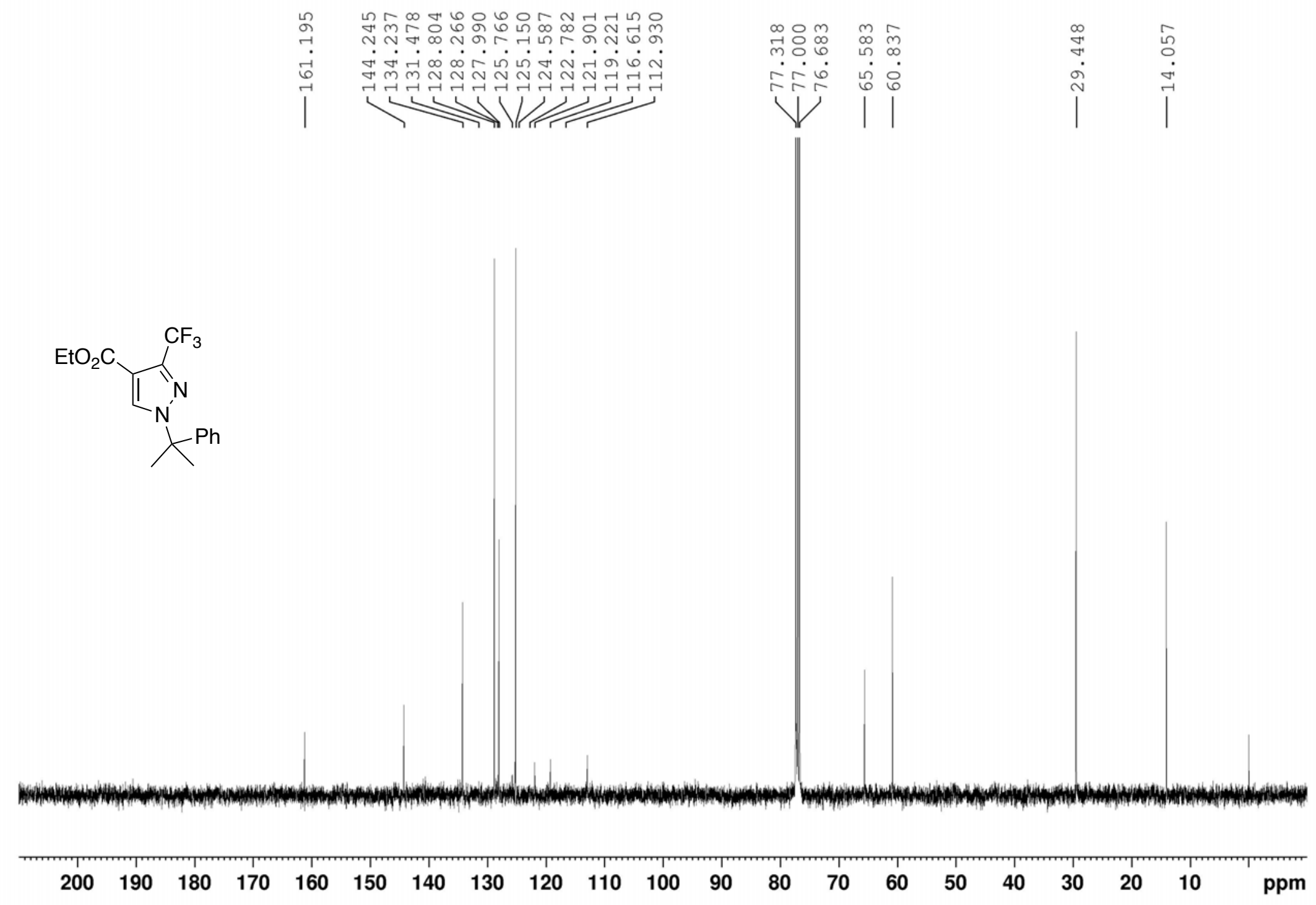

${ }^{13} \mathrm{C}$ NMR spectrum of $\mathbf{3 j b}\left(100.6 \mathrm{MHz}, \mathrm{CDCl}_{3}\right)$ 

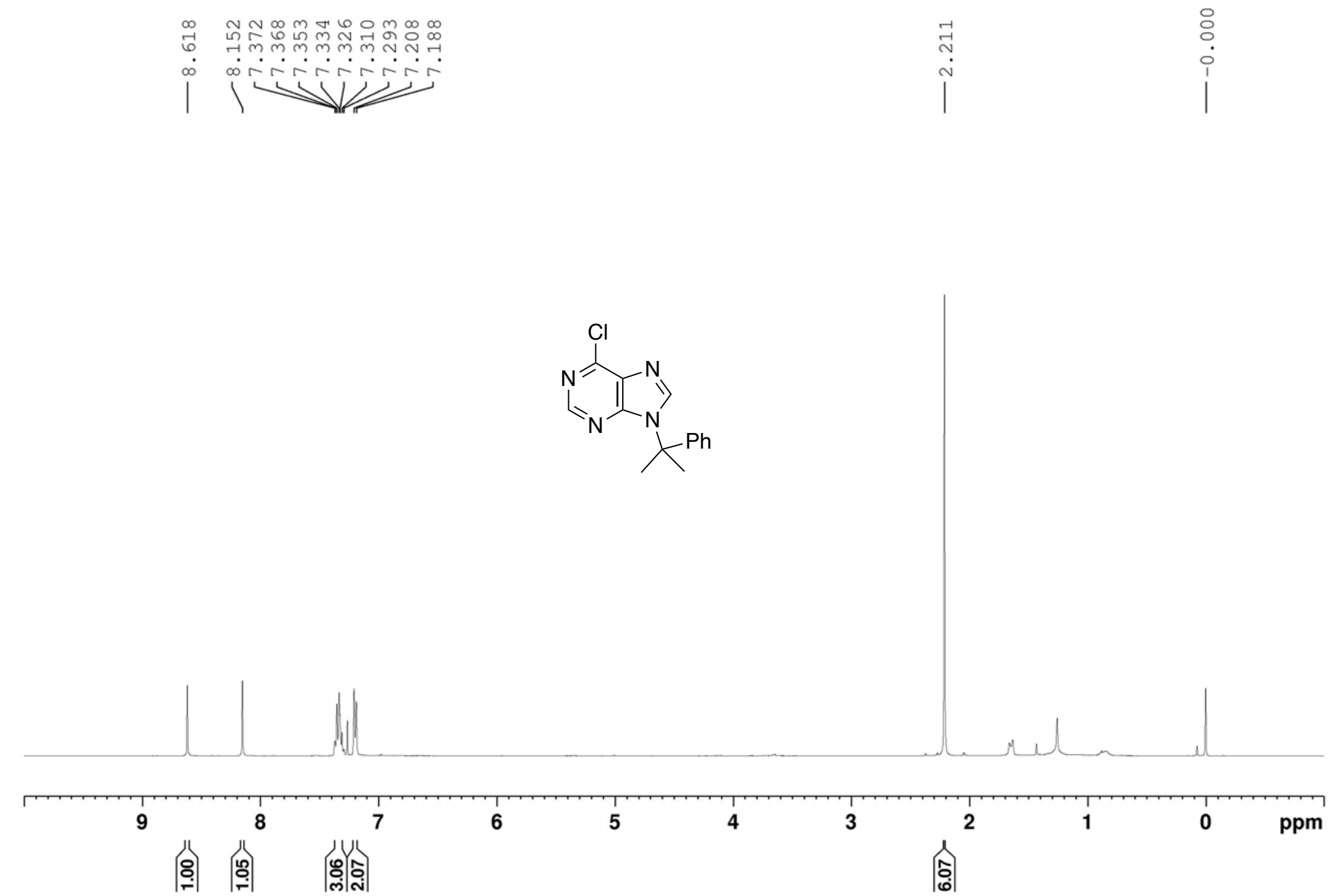

${ }^{1} \mathrm{H}$ NMR spectrum of $\mathbf{3 k b}\left(400 \mathrm{MHz}, \mathrm{CDCl}_{3}\right)$ 


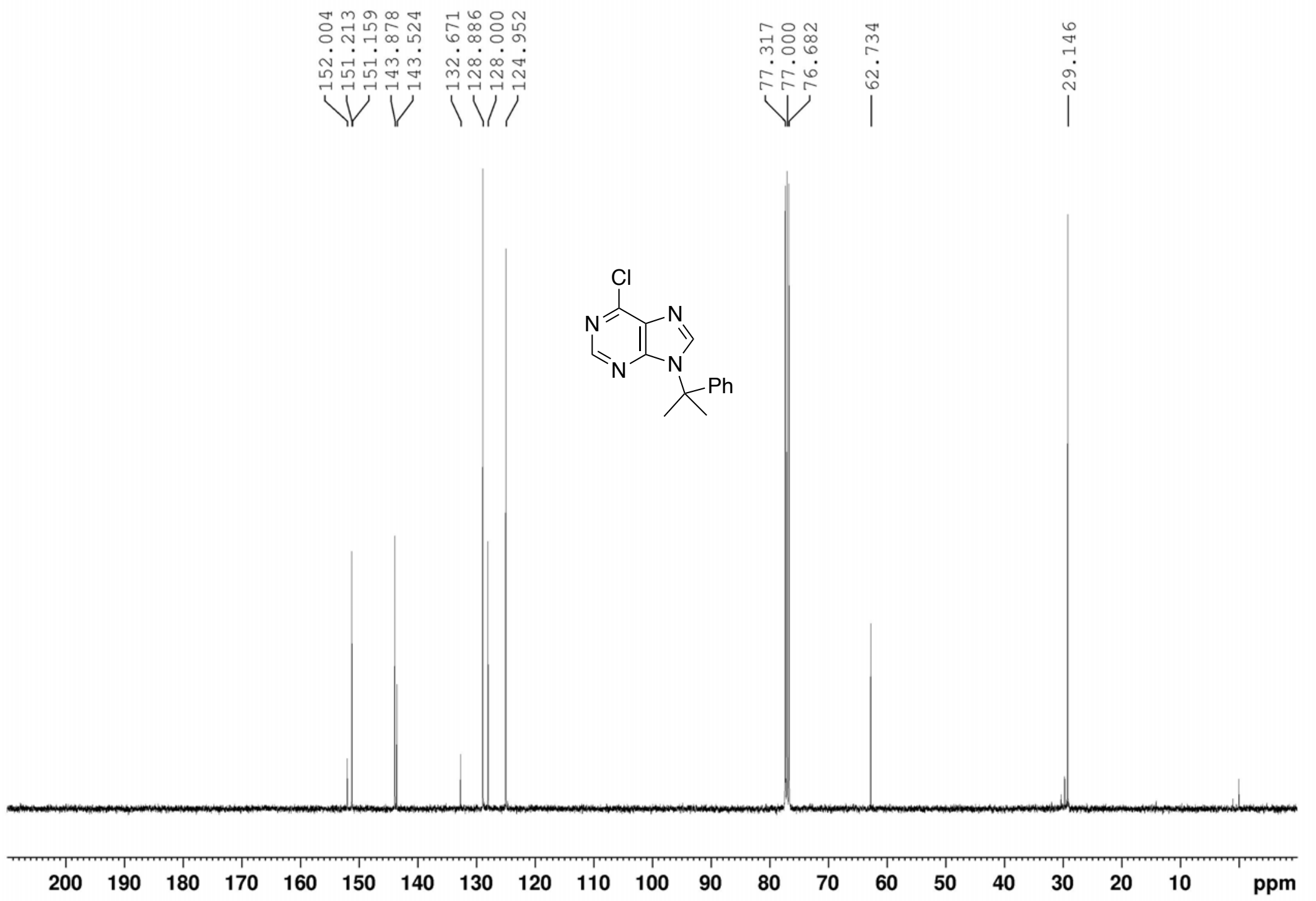

${ }^{13} \mathrm{C}$ NMR spectrum of $\mathbf{3 k b}\left(100.6 \mathrm{MHz}, \mathrm{CDCl}_{3}\right)$ 


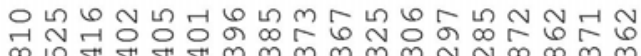

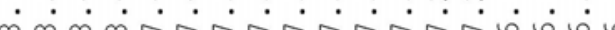

$1 \times$

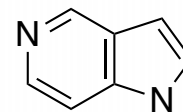

$K^{\mathrm{Ph}}$

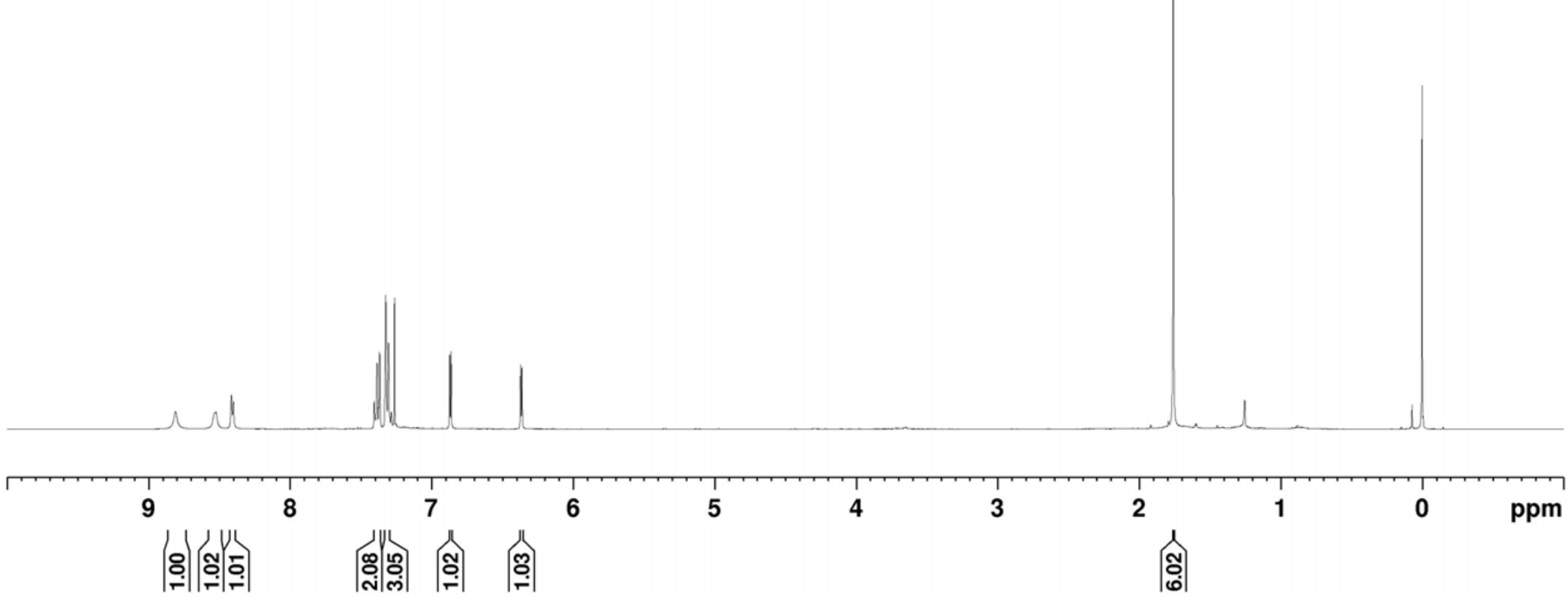

${ }^{1} \mathrm{H}$ NMR spectrum of $\mathbf{3 l b}\left(400 \mathrm{MHz}, \mathrm{CDCl}_{3}\right)$ 


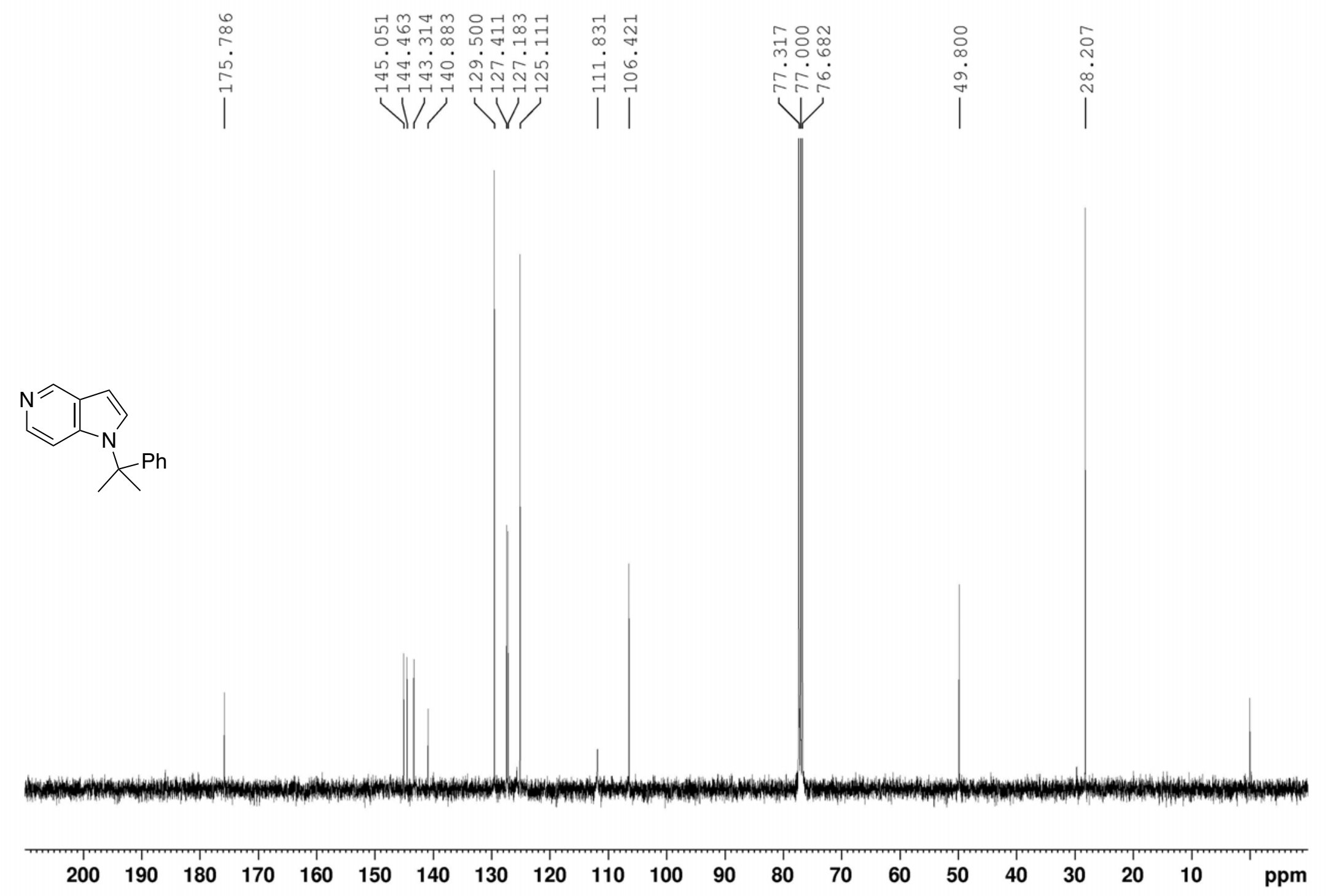

${ }^{13} \mathrm{C}$ NMR spectrum of $\mathbf{3 l b}\left(100.6 \mathrm{MHz}, \mathrm{CDCl}_{3}\right)$

S62 


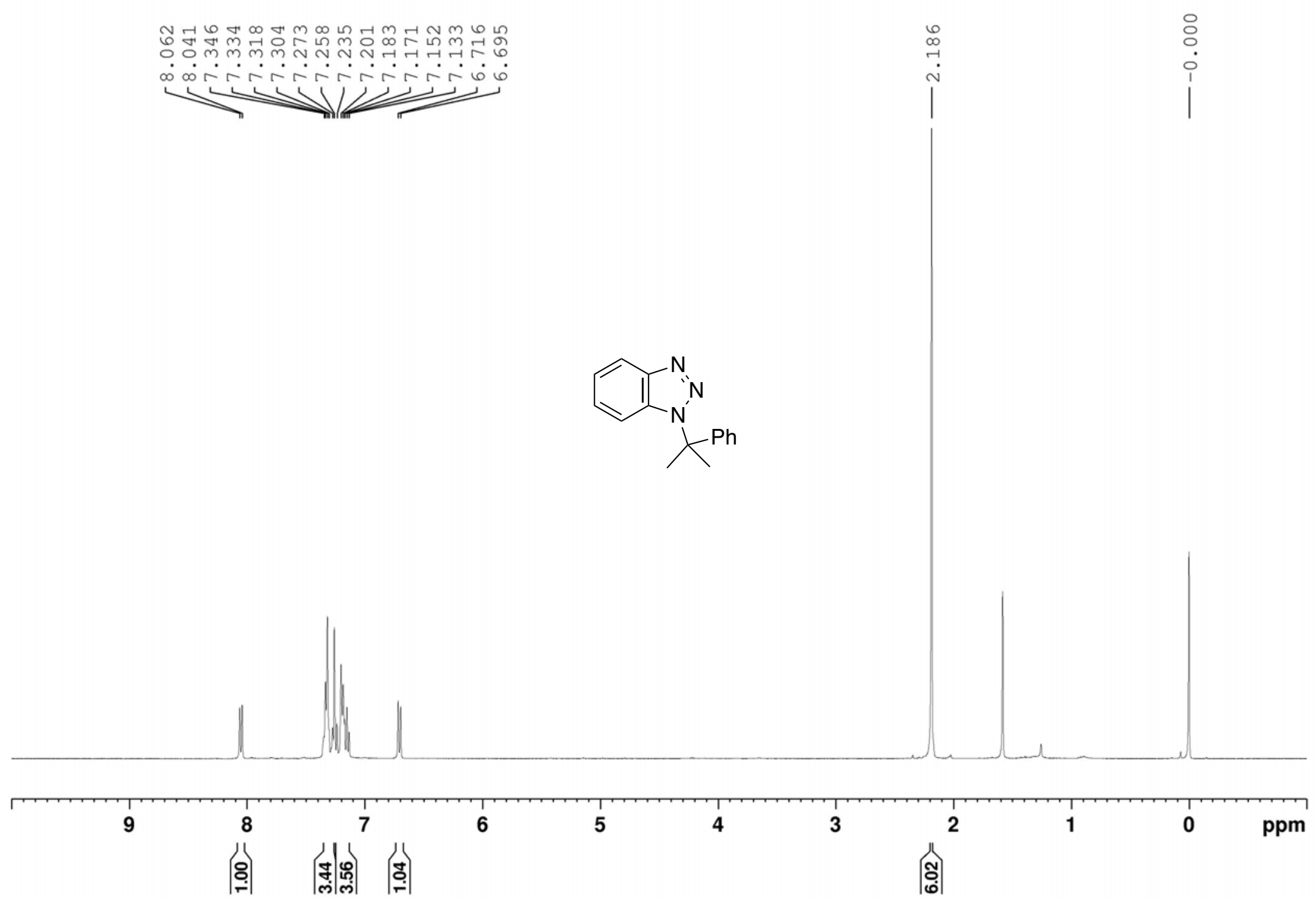

${ }^{1} \mathrm{H}$ NMR spectrum of $\mathbf{3 g b}\left(400 \mathrm{MHz}, \mathrm{CDCl}_{3}\right)$ 


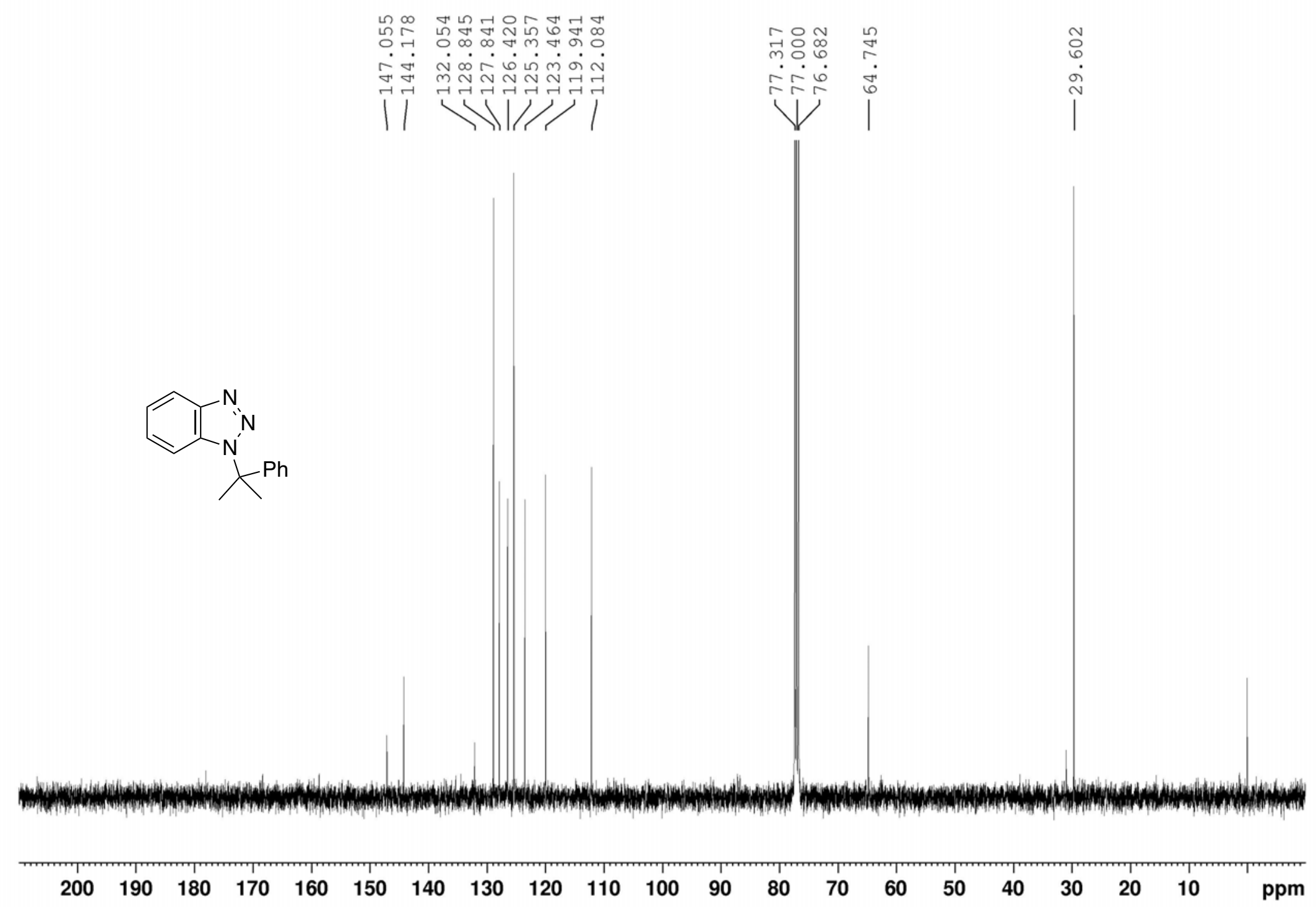

${ }^{13} \mathrm{C}$ NMR spectrum of $\mathbf{3 g b}\left(100.6 \mathrm{MHz}, \mathrm{CDCl}_{3}\right)$ 


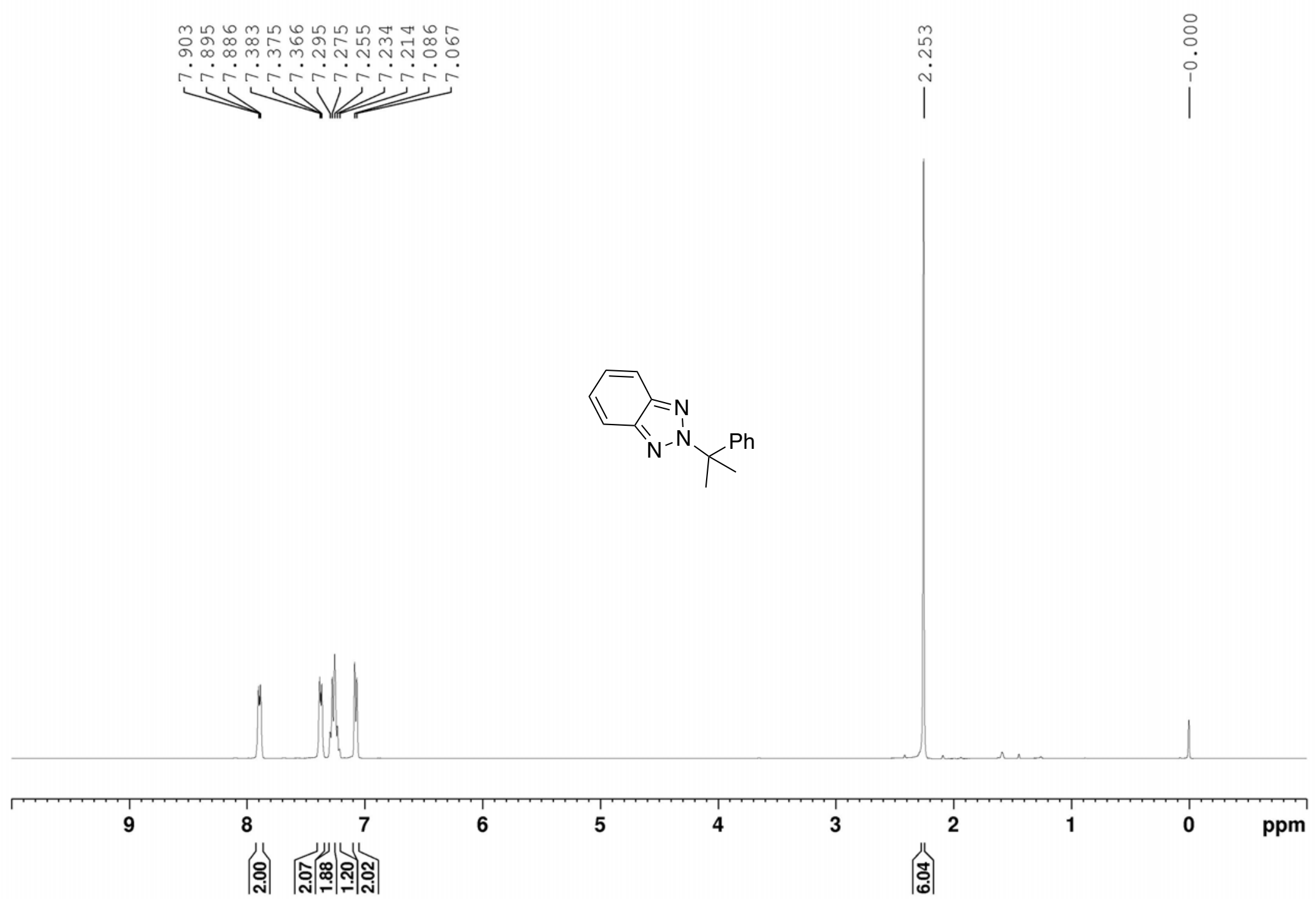

${ }^{1} \mathrm{H}$ NMR spectrum of $\mathbf{3 g b}{ }^{\prime}\left(400 \mathrm{MHz}, \mathrm{CDCl}_{3}\right)$ 


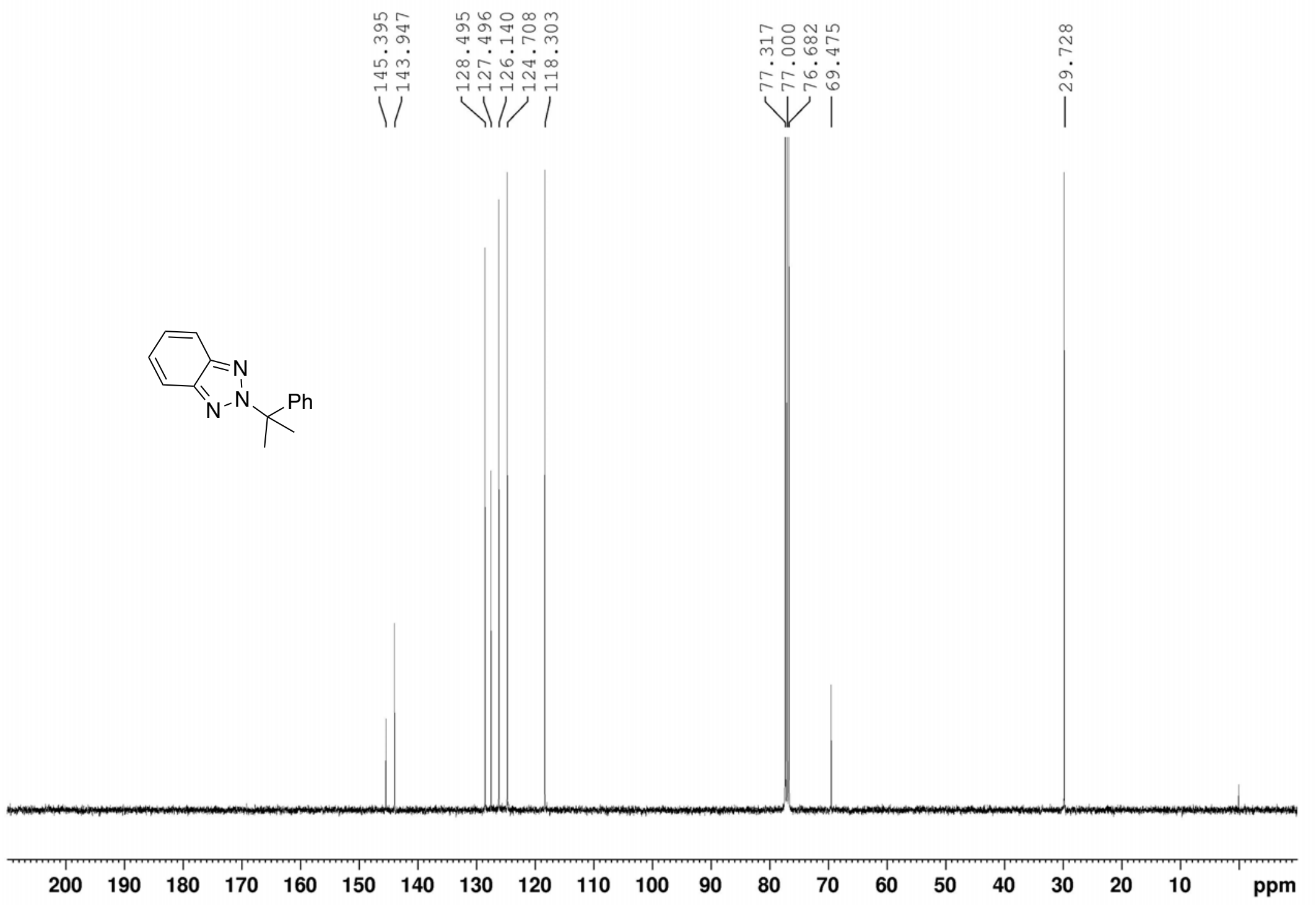

${ }^{13} \mathrm{C}$ NMR spectrum of $\mathbf{3 g b}{ }^{\prime}\left(100.6 \mathrm{MHz}, \mathrm{CDCl}_{3}\right)$ 


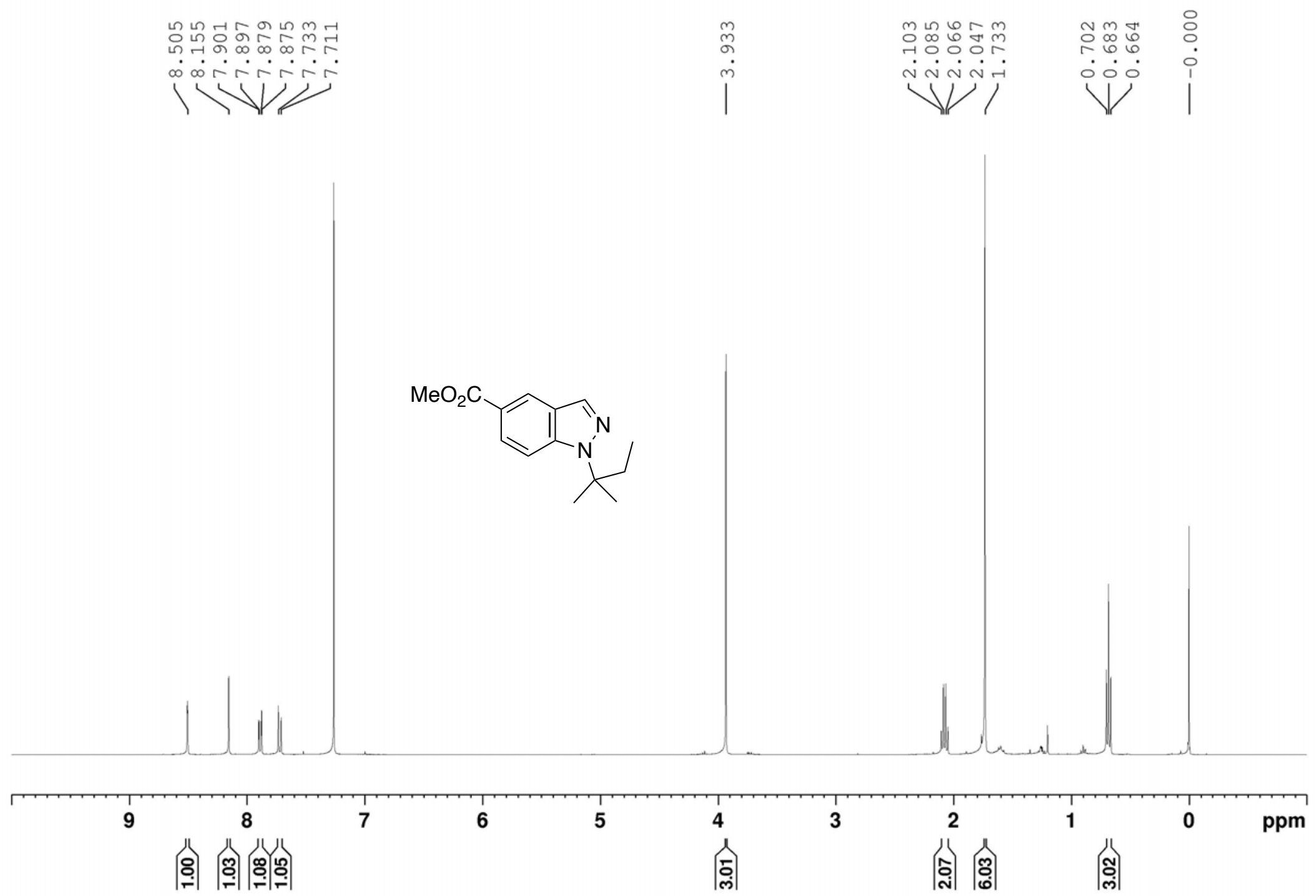

${ }^{1} \mathrm{H}$ NMR spectrum of $\mathbf{3 d c}\left(400 \mathrm{MHz}, \mathrm{CDCl}_{3}\right)$ 


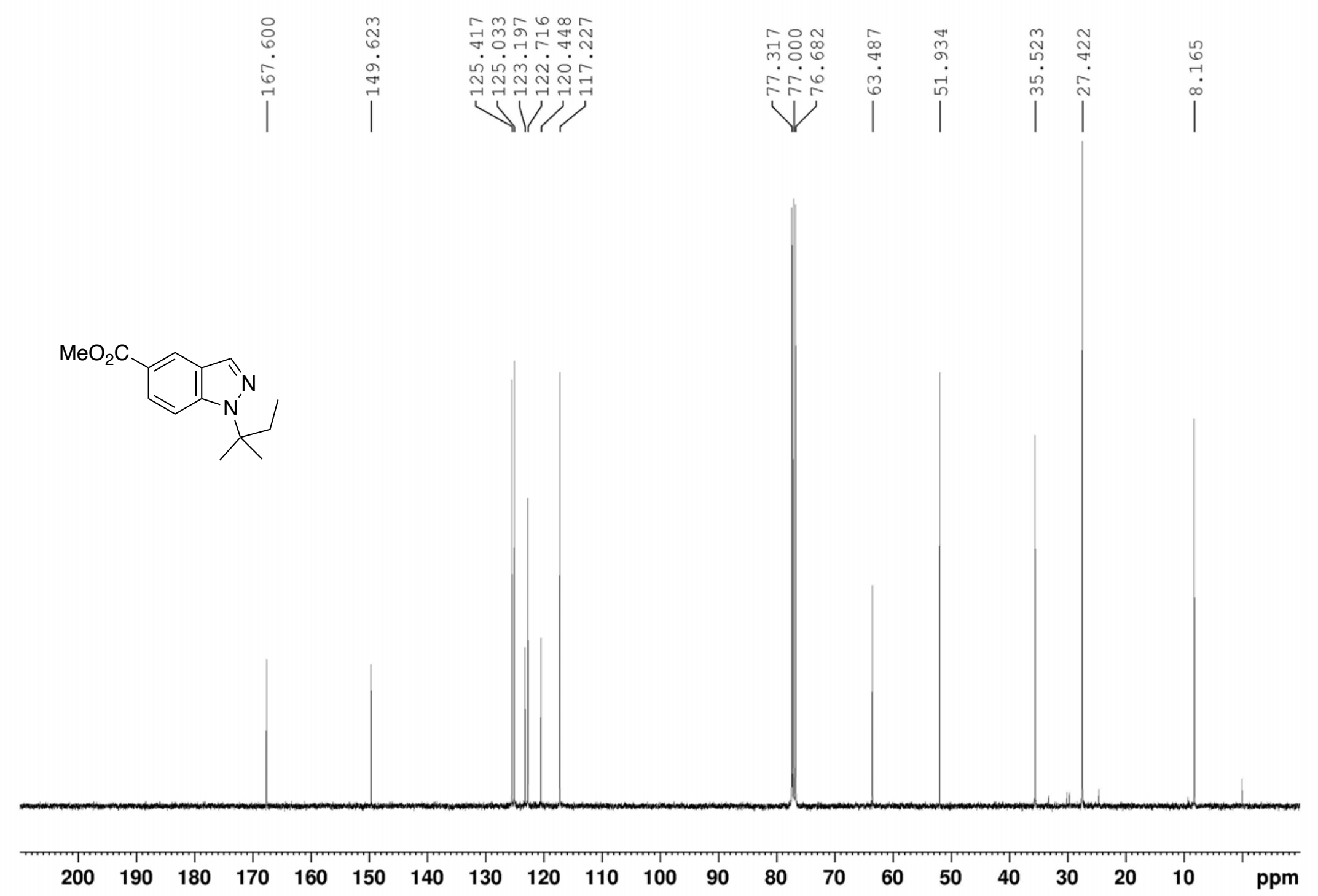

${ }^{13} \mathrm{C}$ NMR spectrum of $\mathbf{3 d c}\left(100.6 \mathrm{MHz}, \mathrm{CDCl}_{3}\right)$ 


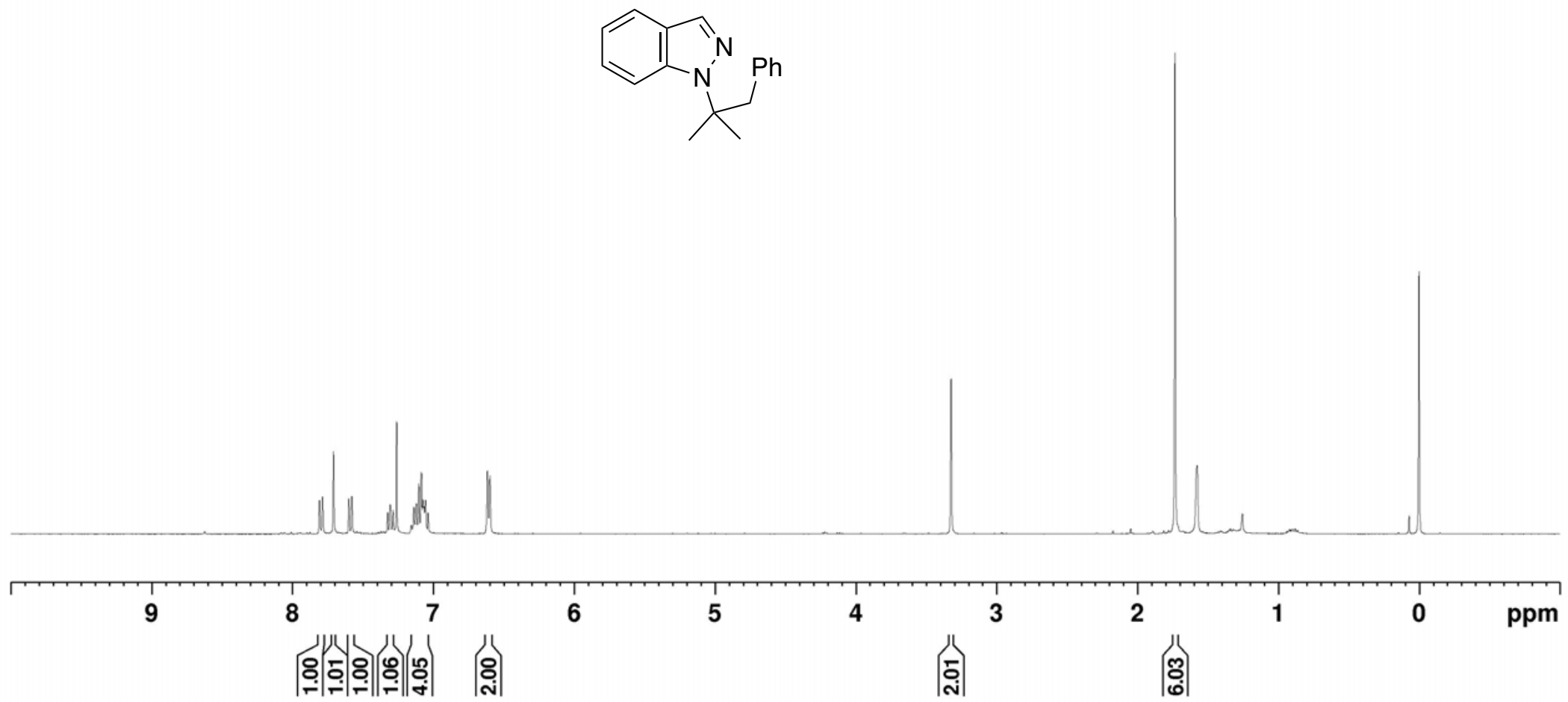

${ }^{1} \mathrm{H}$ NMR spectrum of $\mathbf{3 b d}\left(400 \mathrm{MHz}, \mathrm{CDCl}_{3}\right)$ 


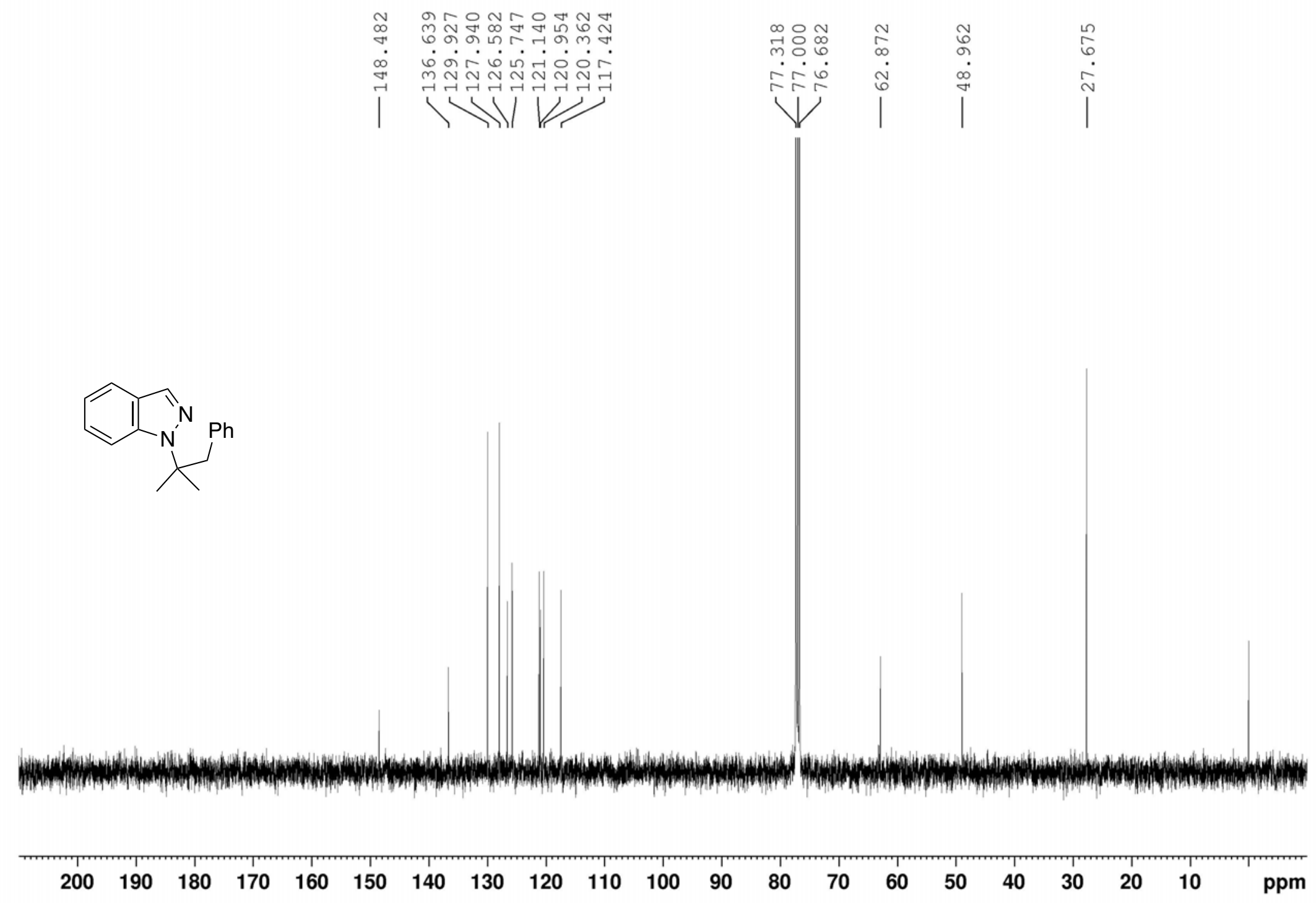

${ }^{13} \mathrm{C}$ NMR spectrum of 3 bd $\left(100.6 \mathrm{MHz}, \mathrm{CDCl}_{3}\right)$ 

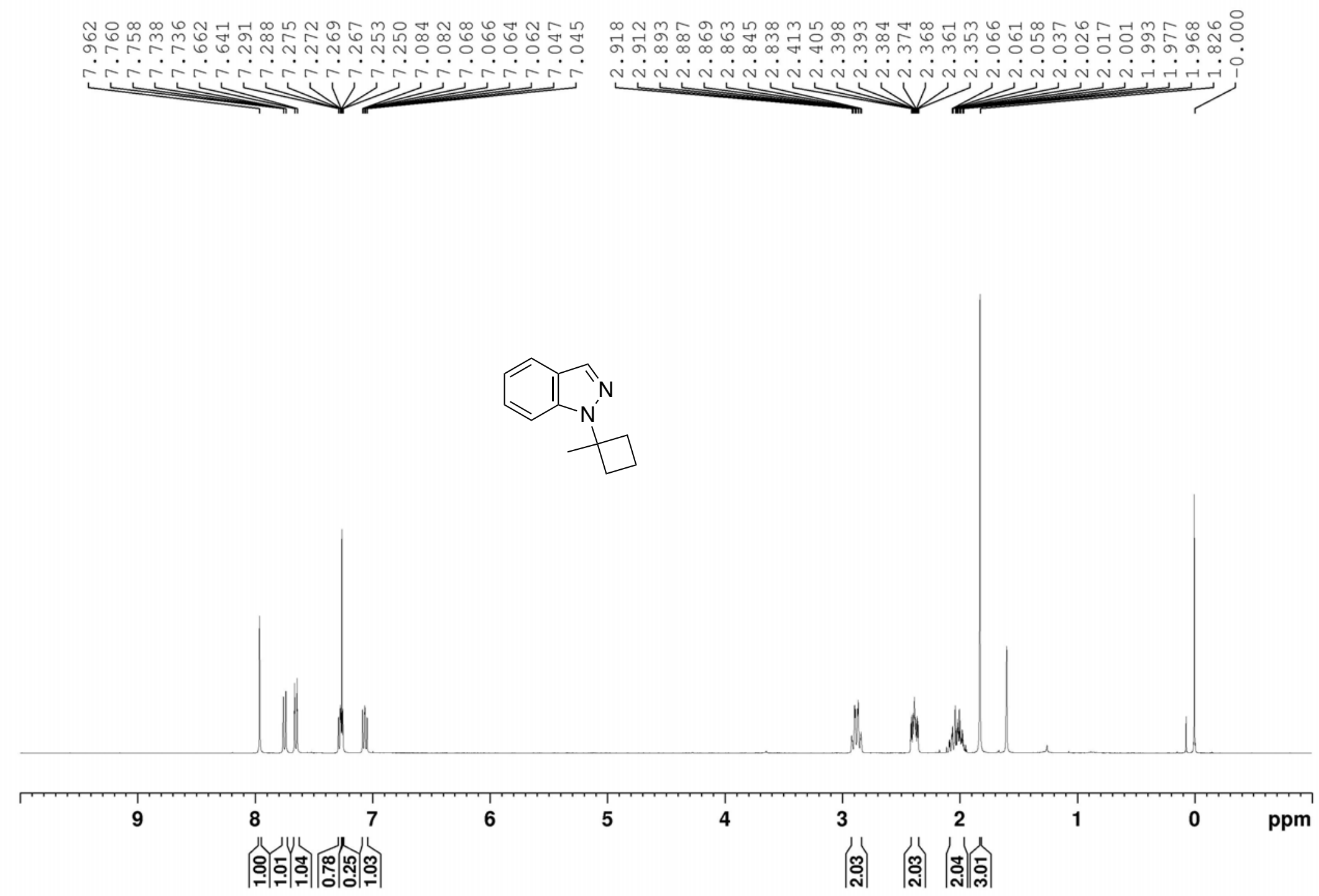

${ }^{1} \mathrm{H}$ NMR spectrum of $3 \mathbf{b e}\left(400 \mathrm{MHz}, \mathrm{CDCl}_{3}\right)$ 


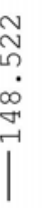

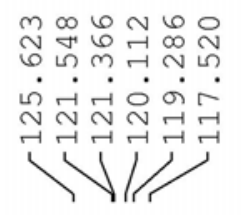

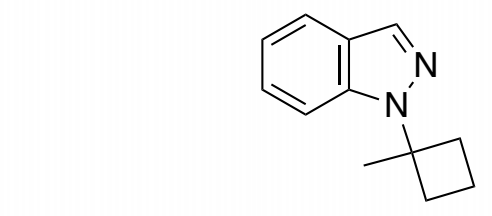

官各

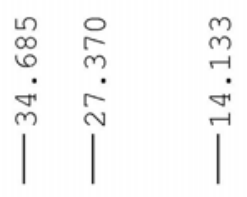

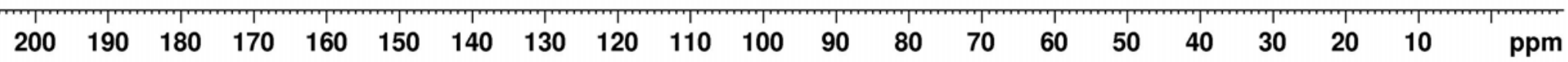

${ }^{13} \mathrm{C}$ NMR spectrum of $3 \mathbf{b e}\left(100.6 \mathrm{MHz}, \mathrm{CDCl}_{3}\right)$

S72 


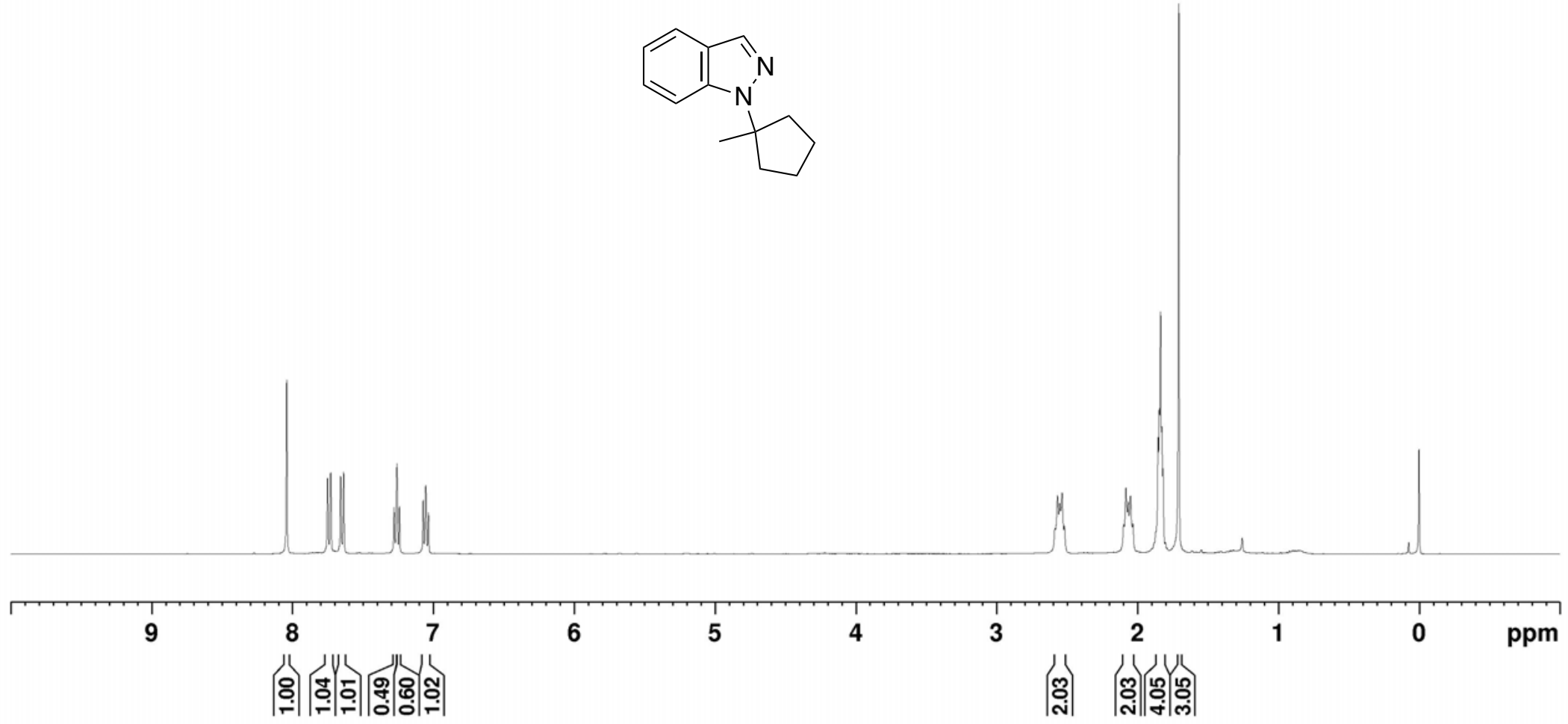

${ }^{1} \mathrm{H}$ NMR spectrum of $\mathbf{3 b f}\left(400 \mathrm{MHz}, \mathrm{CDCl}_{3}\right)$ 


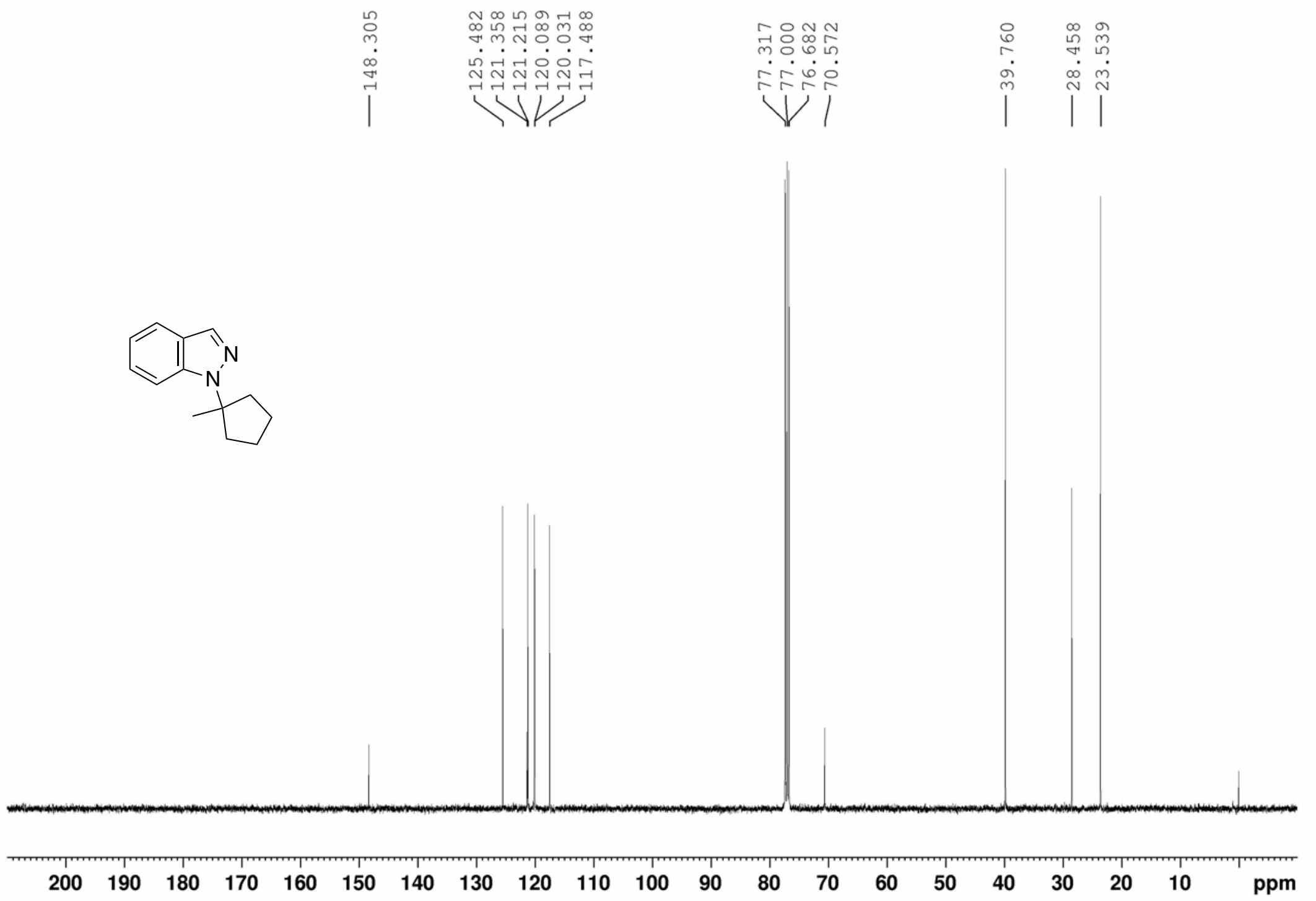

${ }^{13} \mathrm{C}$ NMR spectrum of $\mathbf{3 b f}\left(100.6 \mathrm{MHz}, \mathrm{CDCl}_{3}\right)$ 


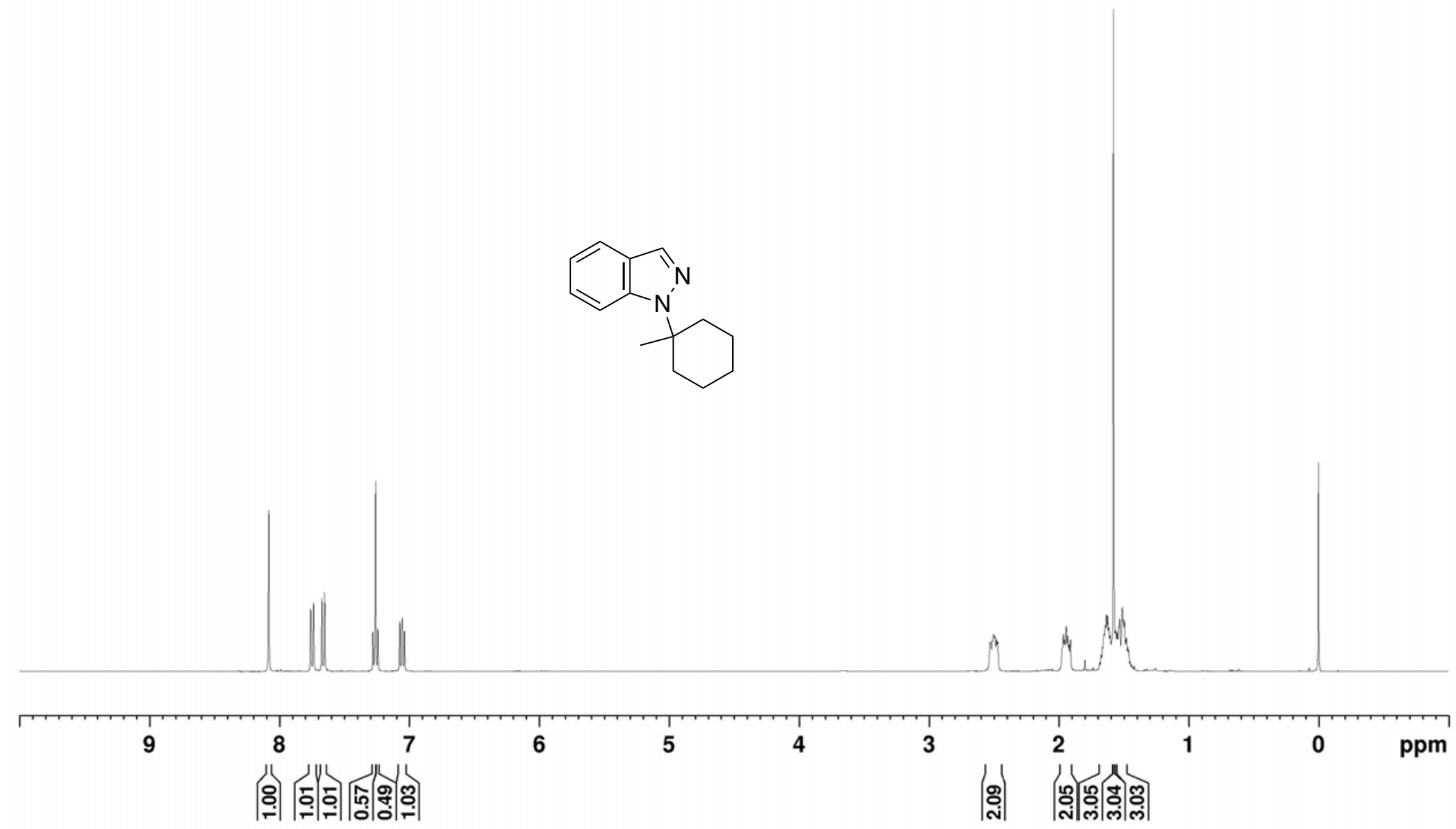

${ }^{1} \mathrm{H}$ NMR spectrum of $\mathbf{3 b g}\left(400 \mathrm{MHz}, \mathrm{CDCl}_{3}\right)$ 


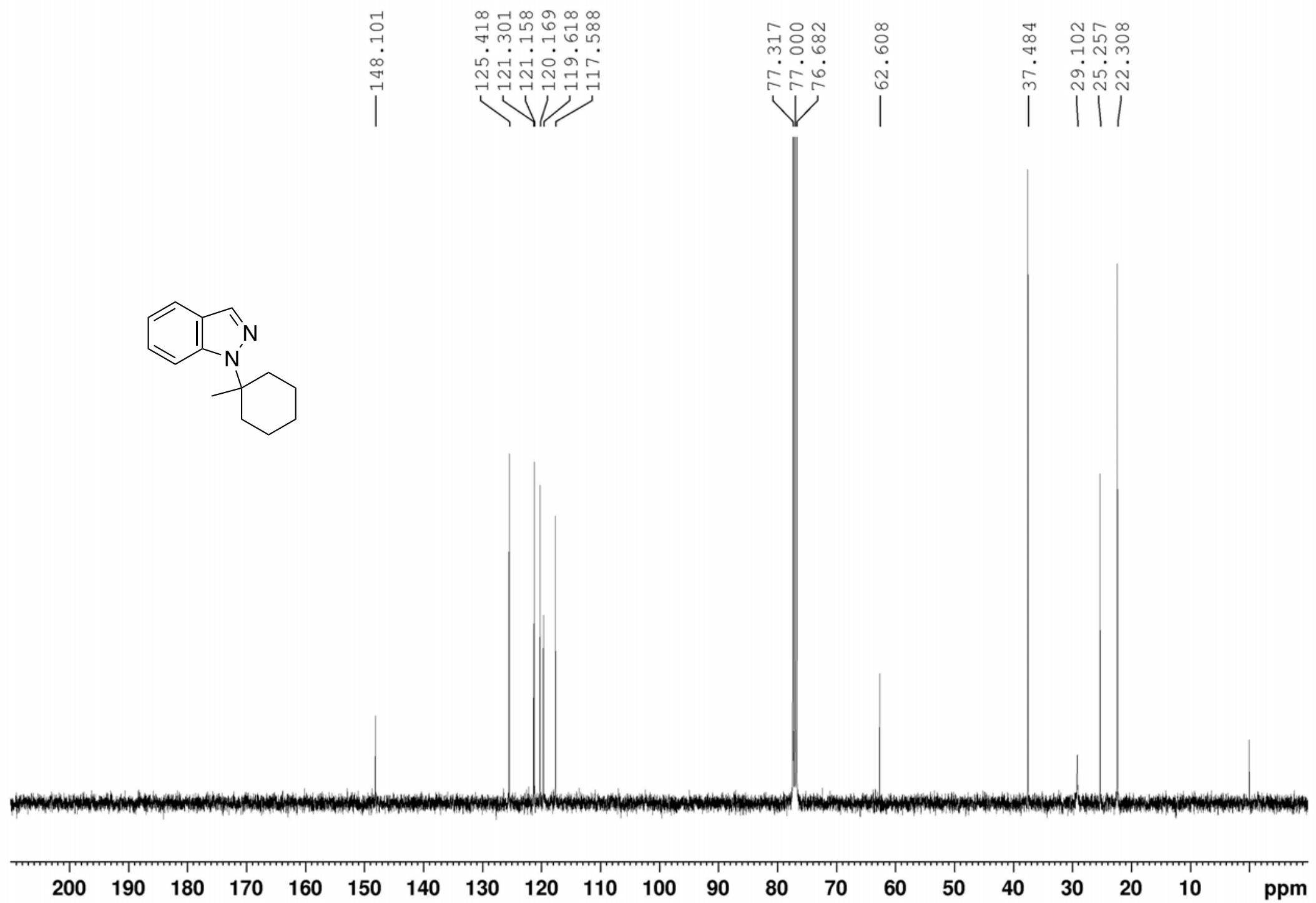

${ }^{13} \mathrm{C}$ NMR spectrum of $\mathbf{3 b g}\left(100.6 \mathrm{MHz}, \mathrm{CDCl}_{3}\right)$ 


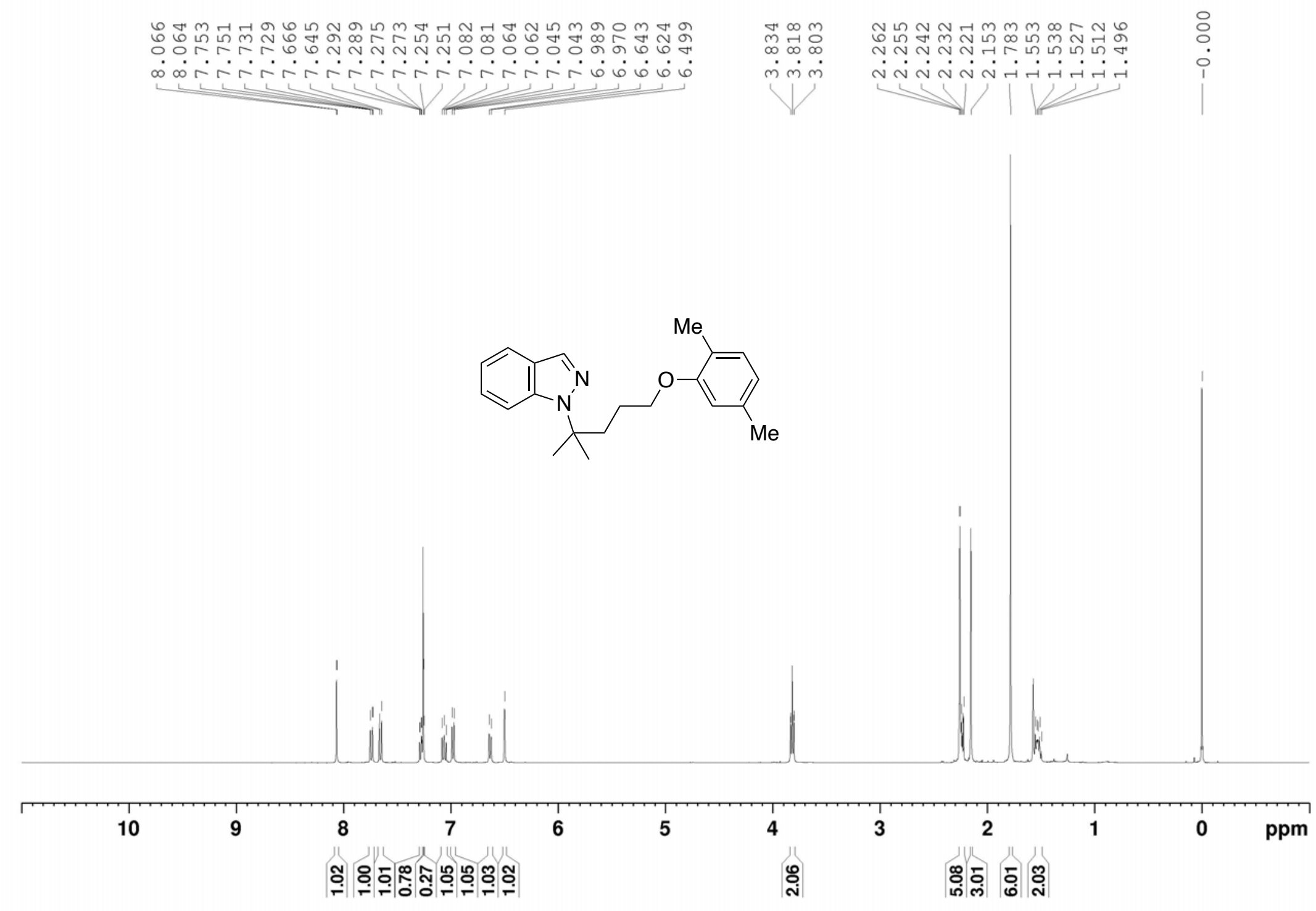

${ }^{1} \mathrm{H}$ NMR spectrum of $\mathbf{3 b h}\left(400 \mathrm{MHz}, \mathrm{CDCl}_{3}\right)$ 


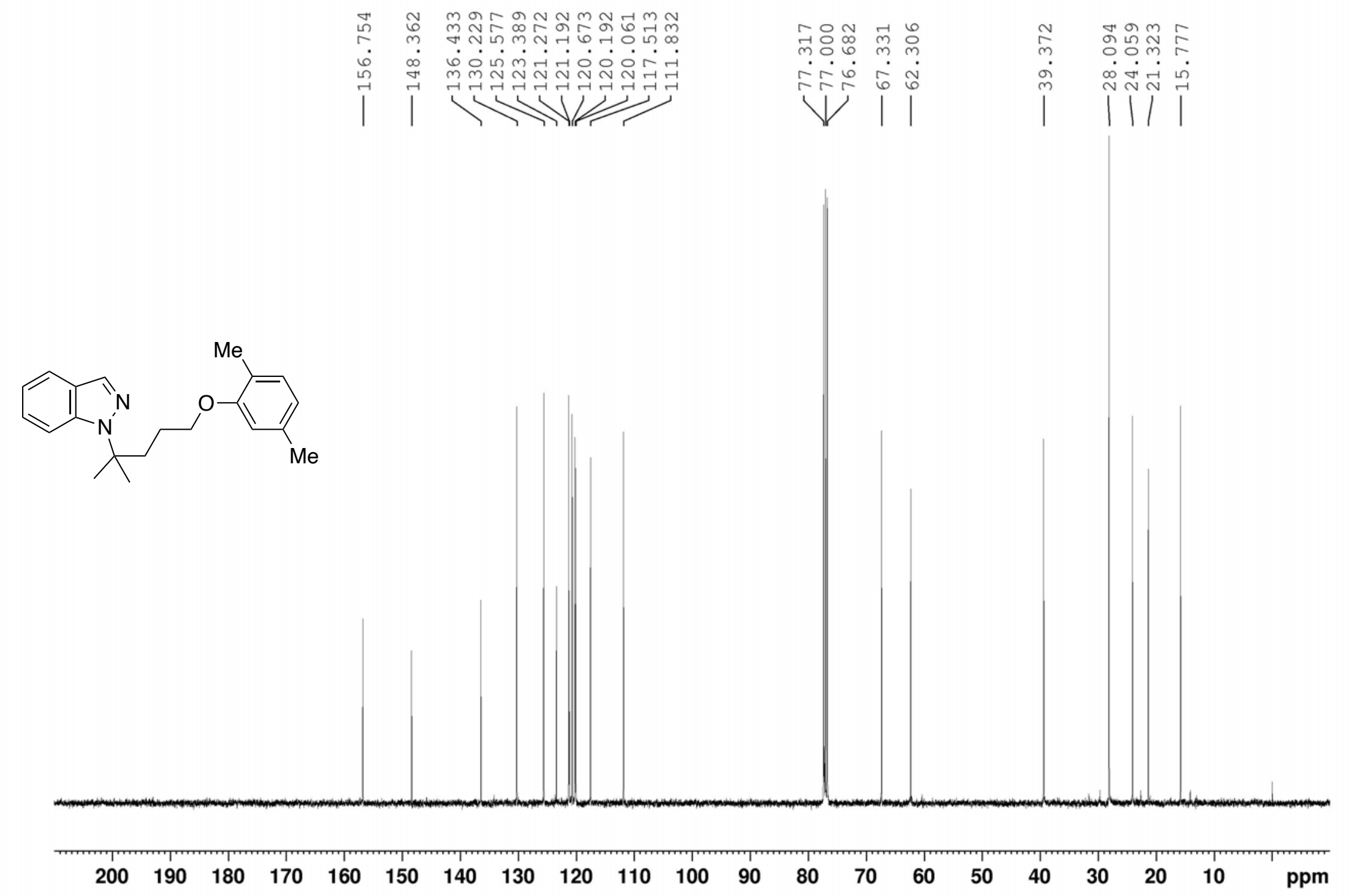

${ }^{13} \mathrm{C}$ NMR spectrum of $\mathbf{3 b h}\left(100.6 \mathrm{MHz}, \mathrm{CDCl}_{3}\right)$ 


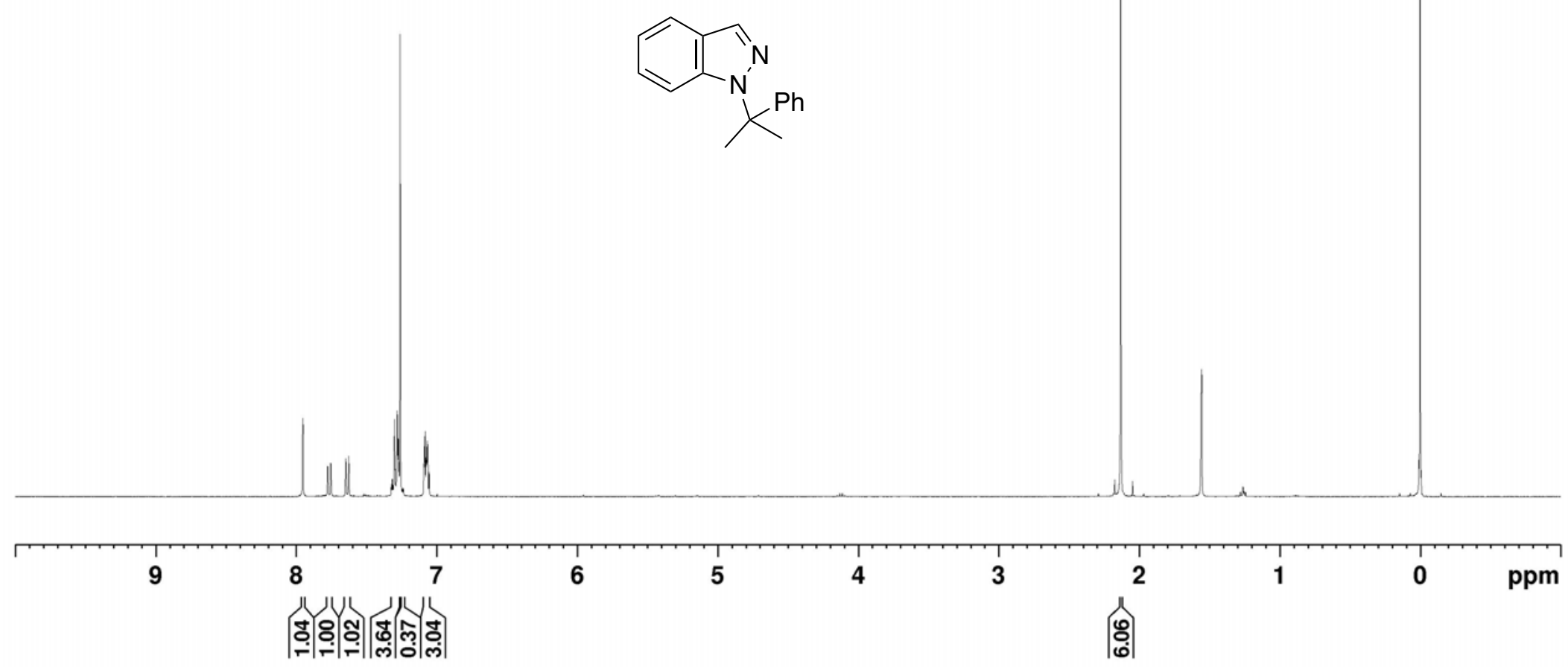

${ }^{1} \mathrm{H}$ NMR spectrum of $\mathbf{3 b b}\left(400 \mathrm{MHz}, \mathrm{CDCl}_{3}\right)$ 


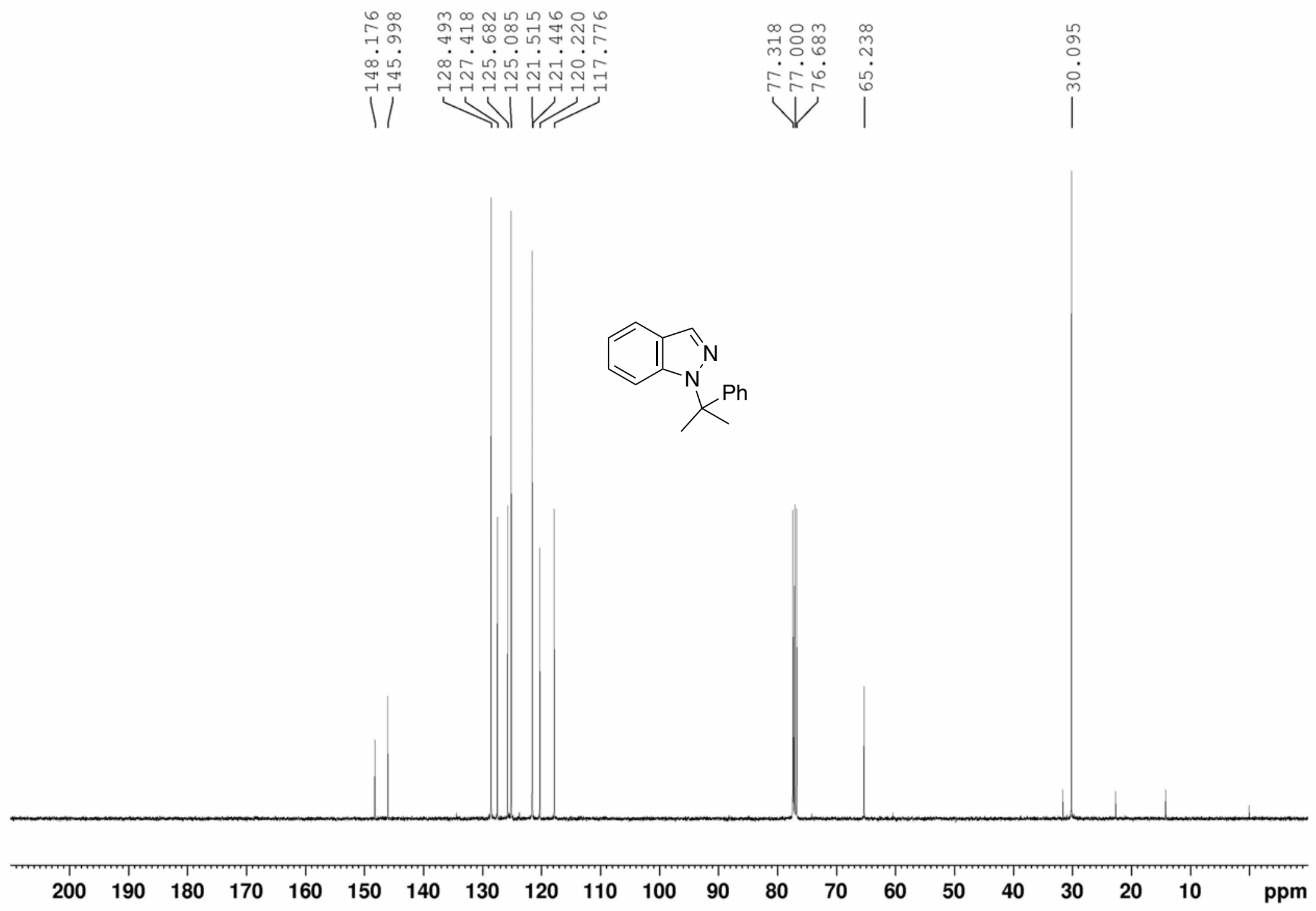

${ }^{13} \mathrm{C}$ NMR spectrum of $\mathbf{3 b b}\left(100.6 \mathrm{MHz}, \mathrm{CDCl}_{3}\right)$ 


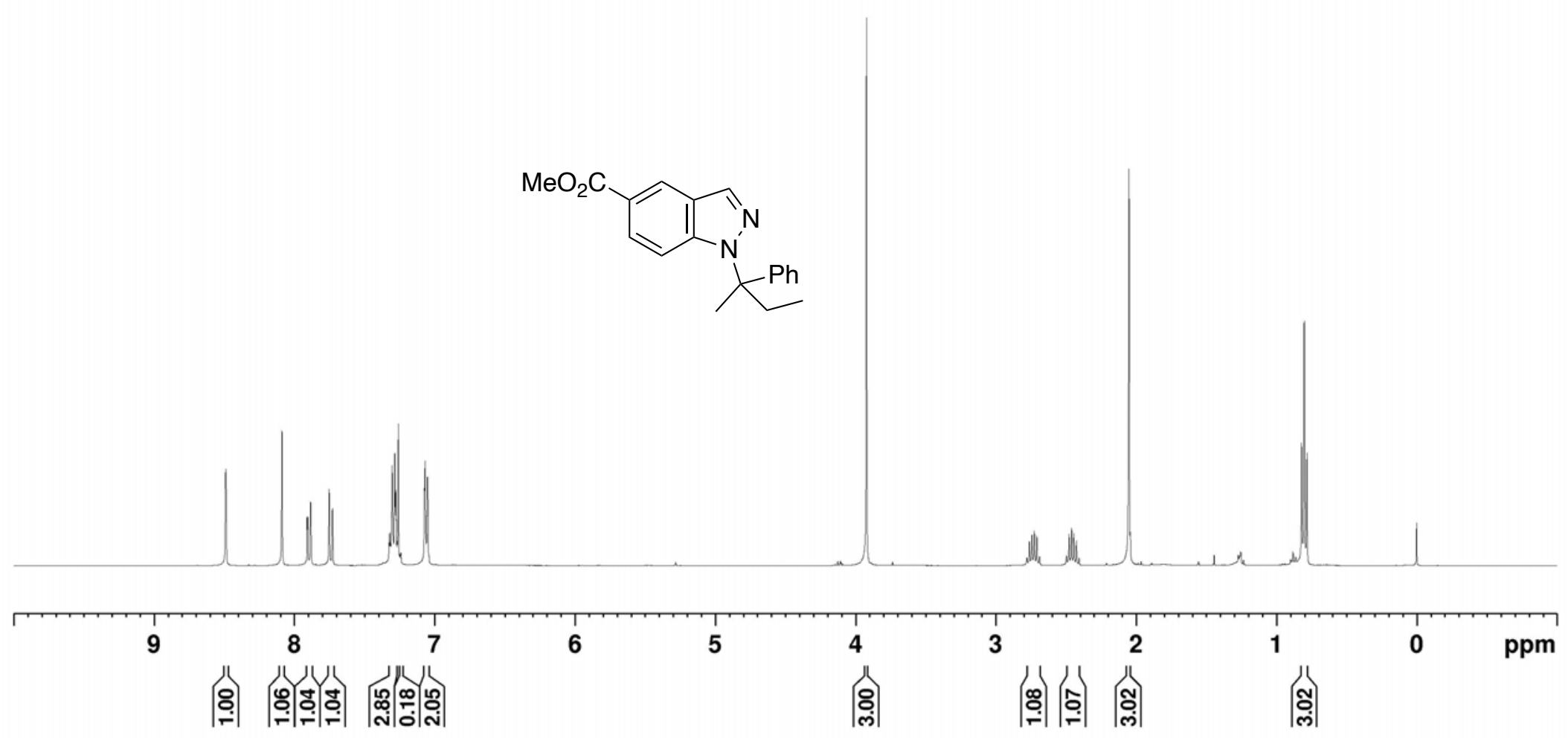

${ }^{1} \mathrm{H}$ NMR spectrum of 3 di $\left(400 \mathrm{MHz}, \mathrm{CDCl}_{3}\right)$ 


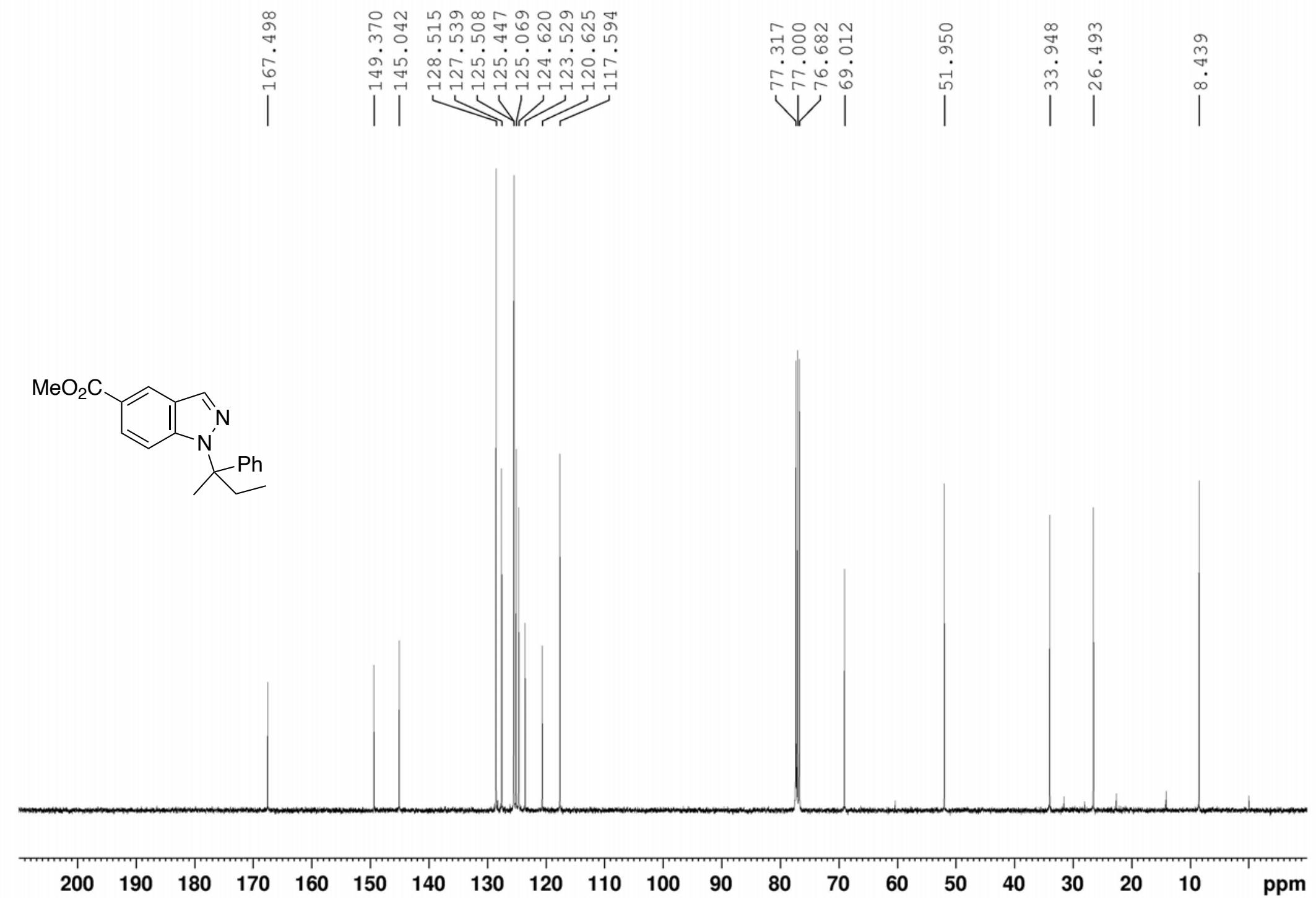

${ }^{13} \mathrm{C}$ NMR spectrum of $\mathbf{3 d i}\left(100.6 \mathrm{MHz}, \mathrm{CDCl}_{3}\right)$ 
જூ mmmmmmmmmm $+1$

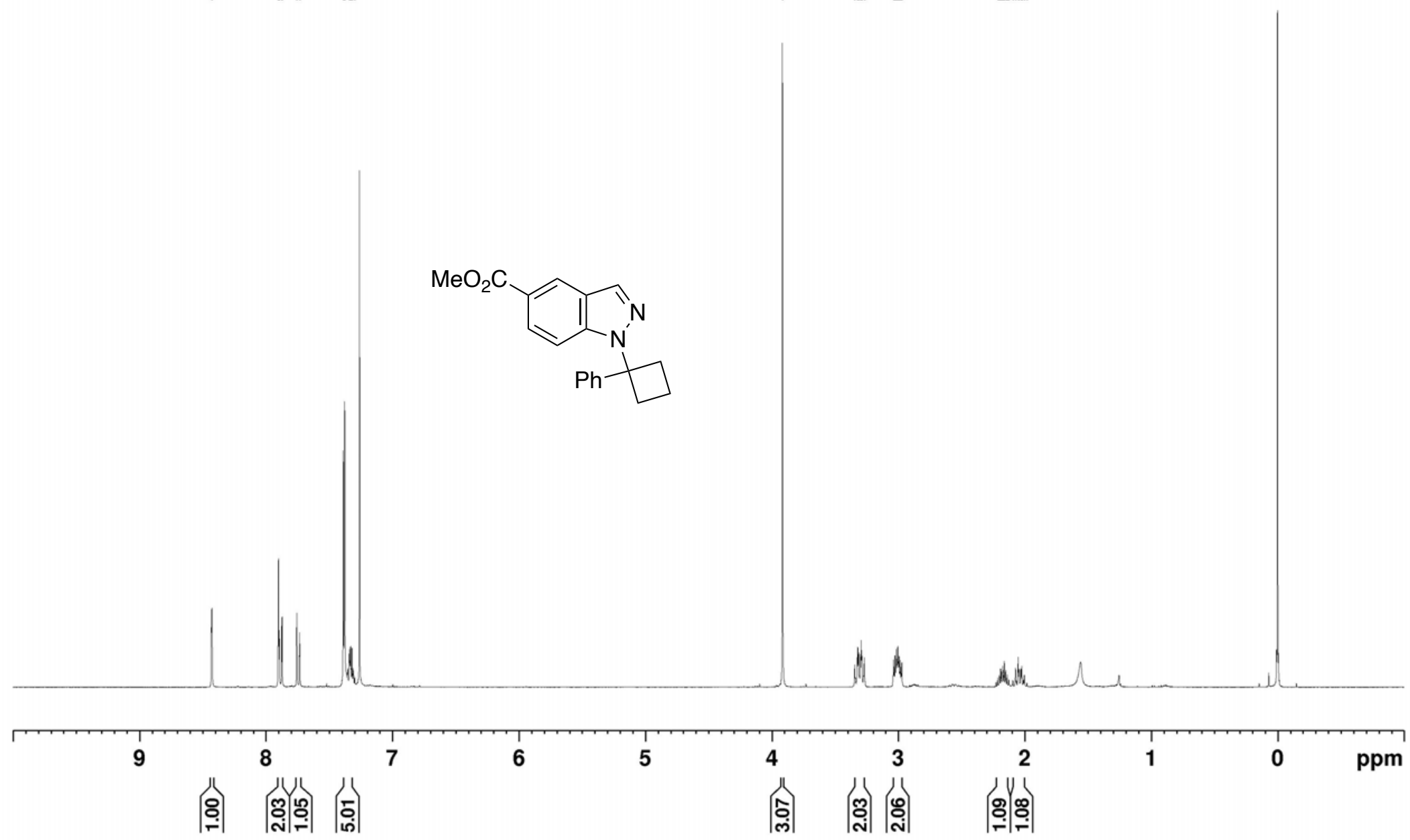

${ }^{1} \mathrm{H}$ NMR spectrum of $\mathbf{3 d j}\left(400 \mathrm{MHz}, \mathrm{CDCl}_{3}\right)$ 


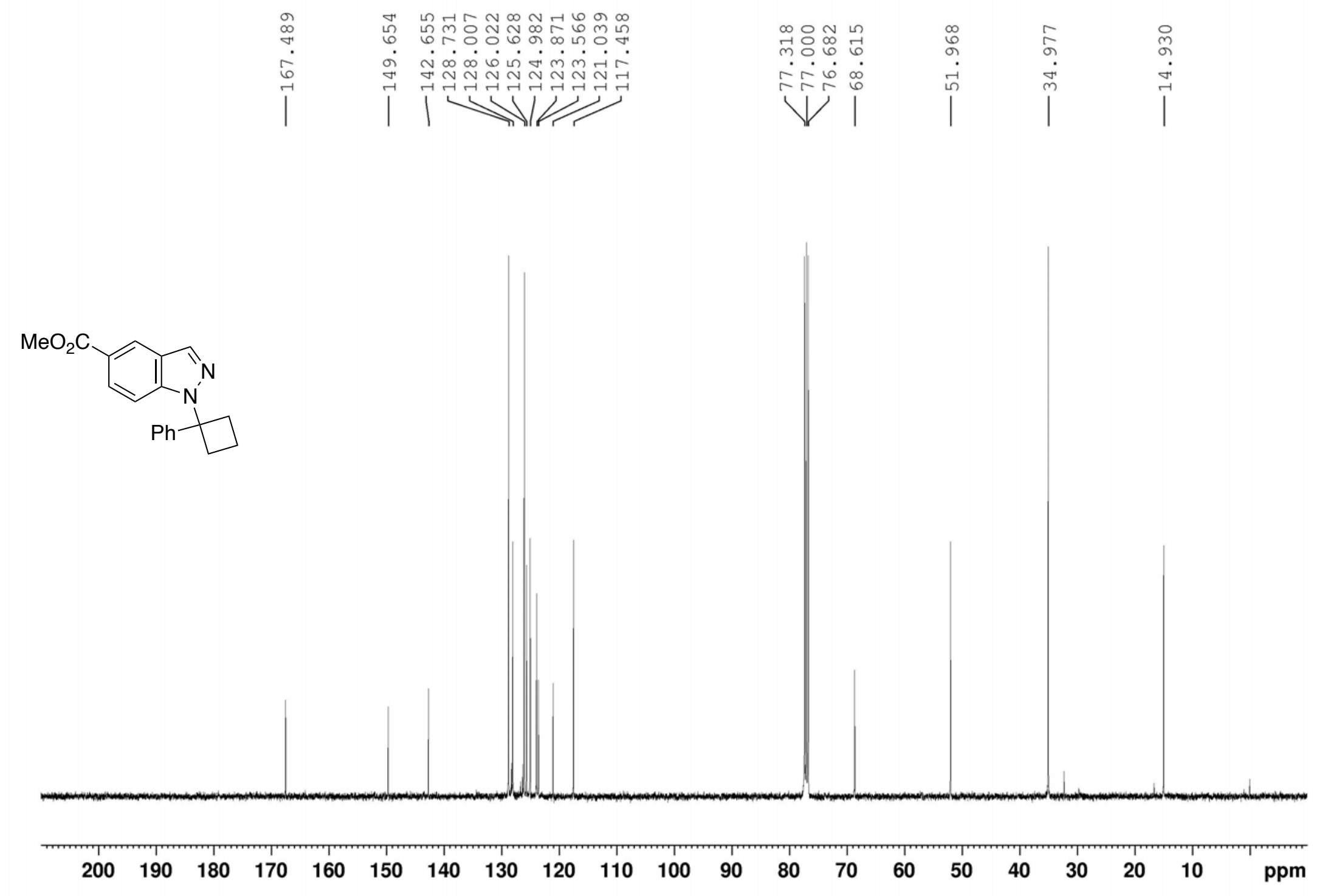

${ }^{13} \mathrm{C}$ NMR spectrum of $\mathbf{3 d j}\left(100.6 \mathrm{MHz}, \mathrm{CDCl}_{3}\right)$ 


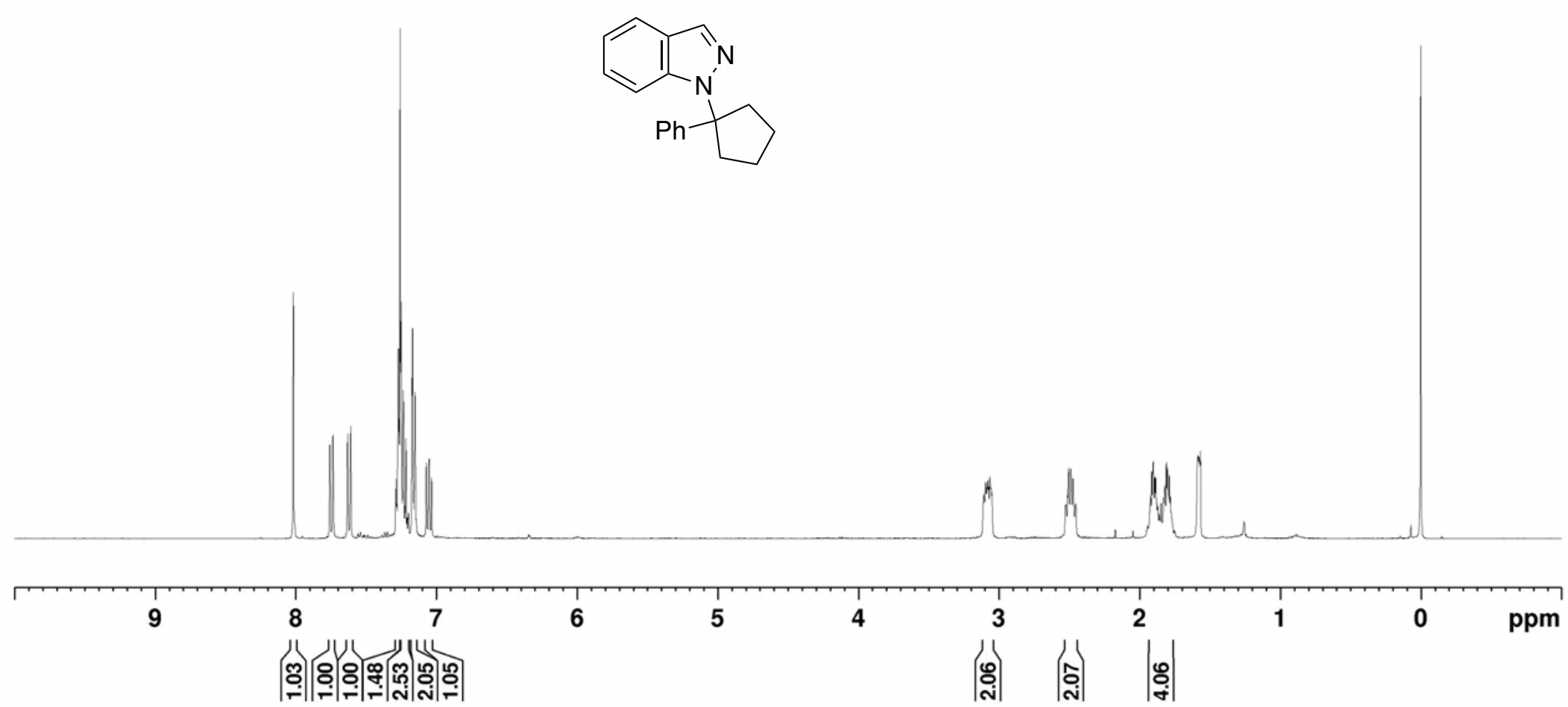

${ }^{1} \mathrm{H}$ NMR spectrum of $\mathbf{3 b k}\left(400 \mathrm{MHz}, \mathrm{CDCl}_{3}\right)$ 


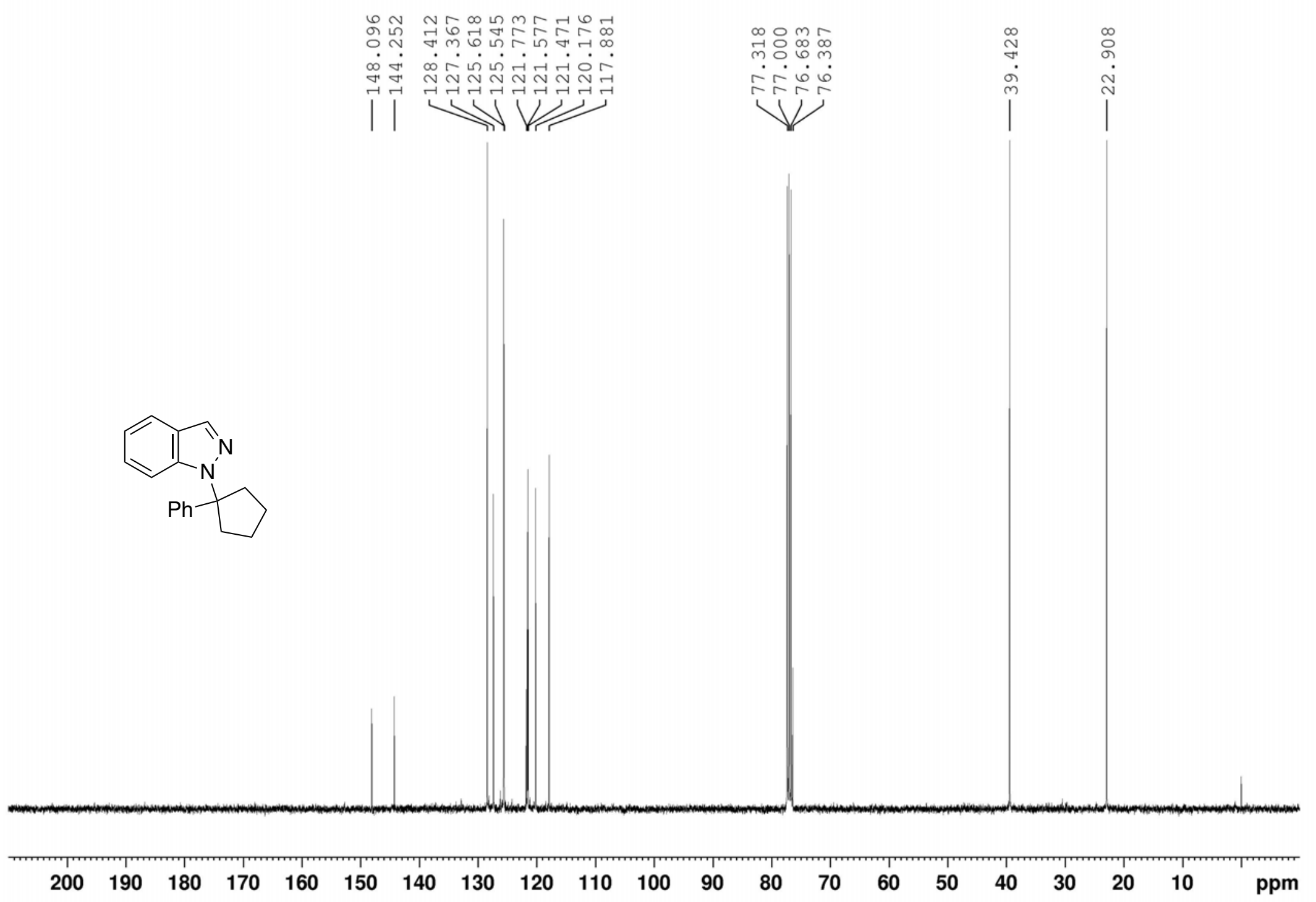

${ }^{13} \mathrm{C}$ NMR spectrum of 3bk $\left(100.6 \mathrm{MHz}, \mathrm{CDCl}_{3}\right)$ 


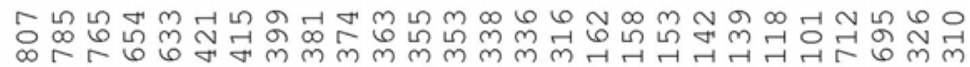

. . . . . .

m

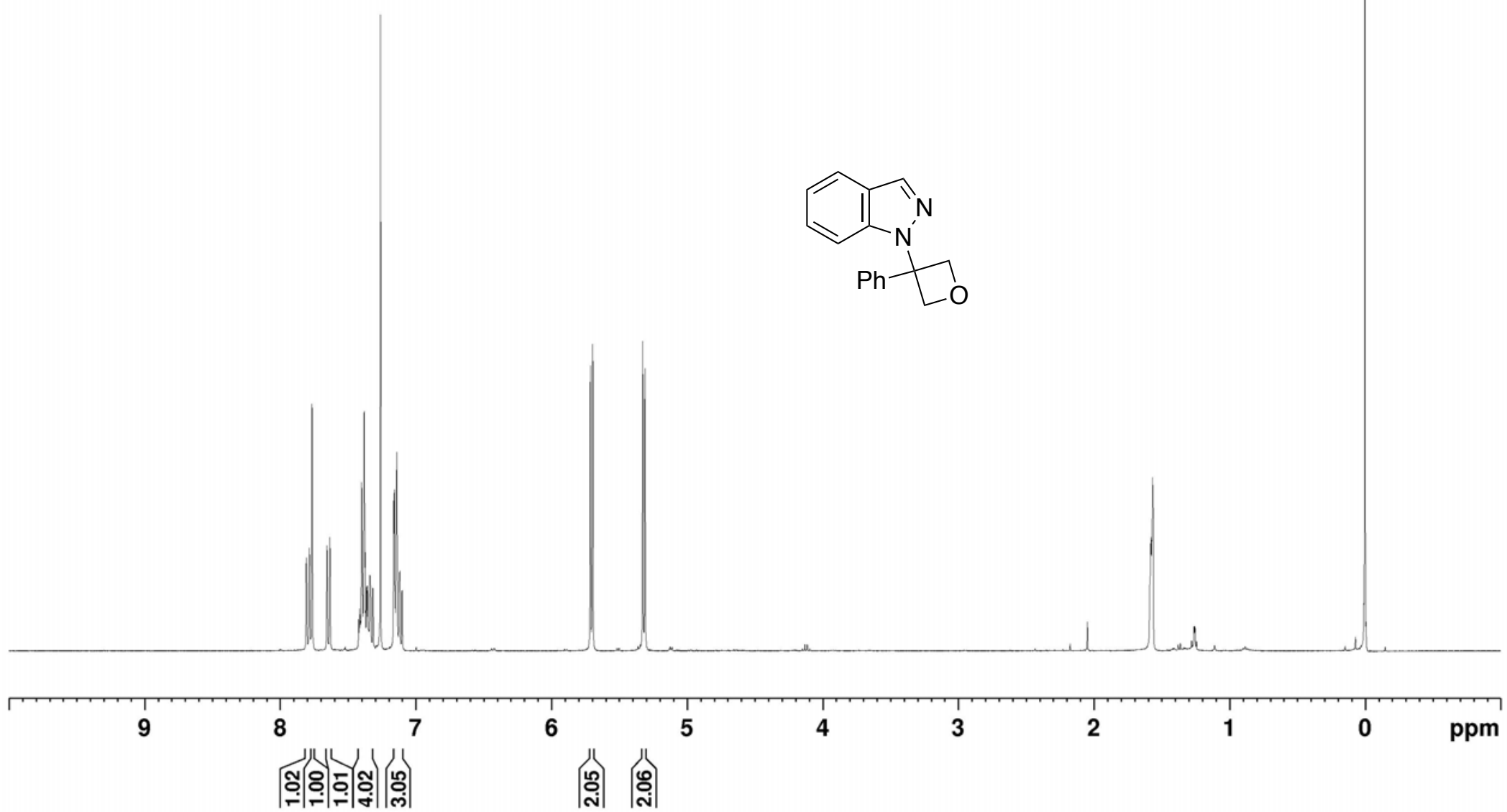

${ }^{1} \mathrm{H}$ NMR spectrum of $\mathbf{3 b l}\left(400 \mathrm{MHz}, \mathrm{CDCl}_{3}\right)$ 


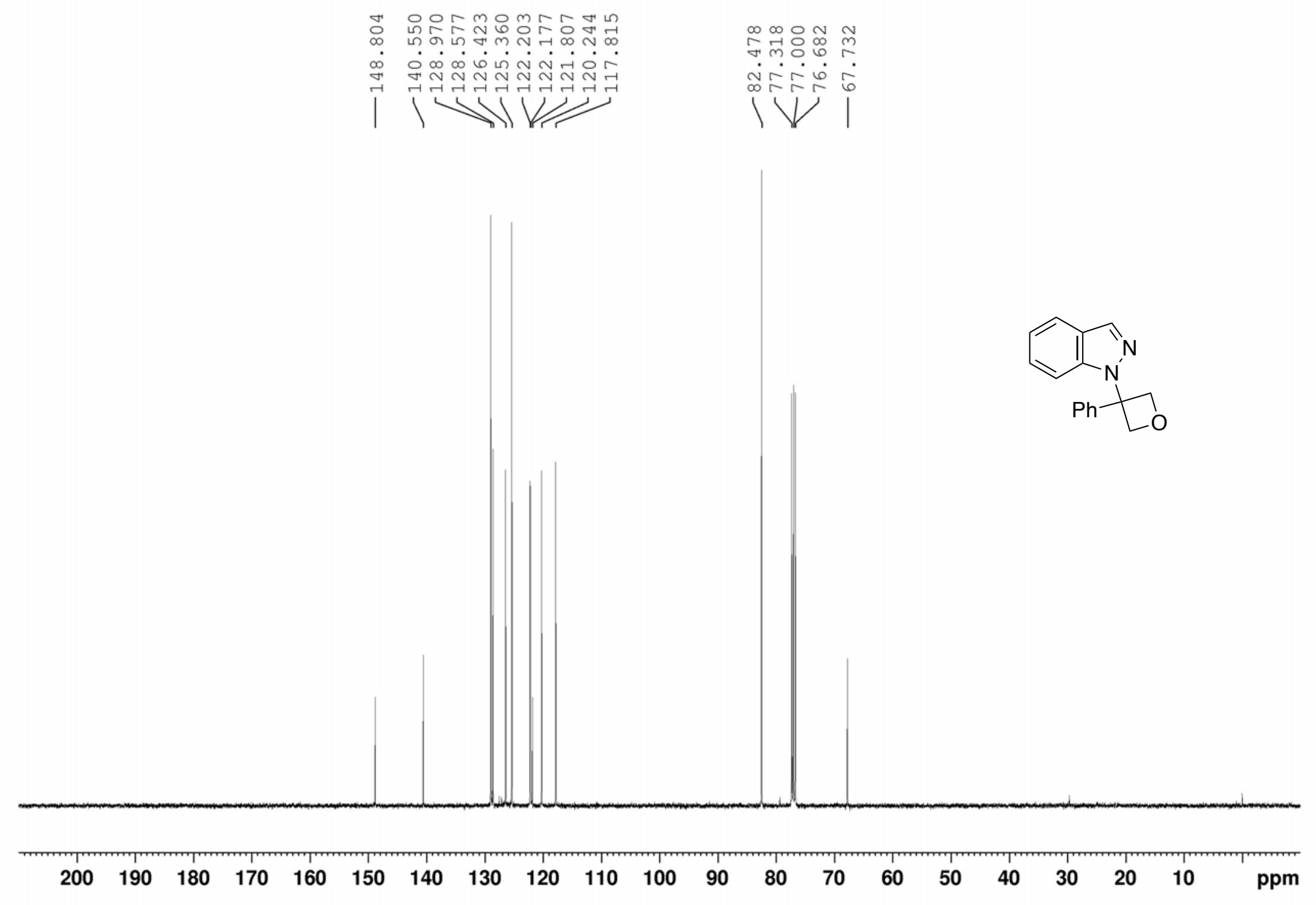

${ }^{13} \mathrm{C}$ NMR spectrum of $\mathbf{3 b l}\left(100.6 \mathrm{MHz}, \mathrm{CDCl}_{3}\right)$ 


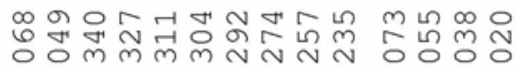

.

$\underbrace{\infty}$

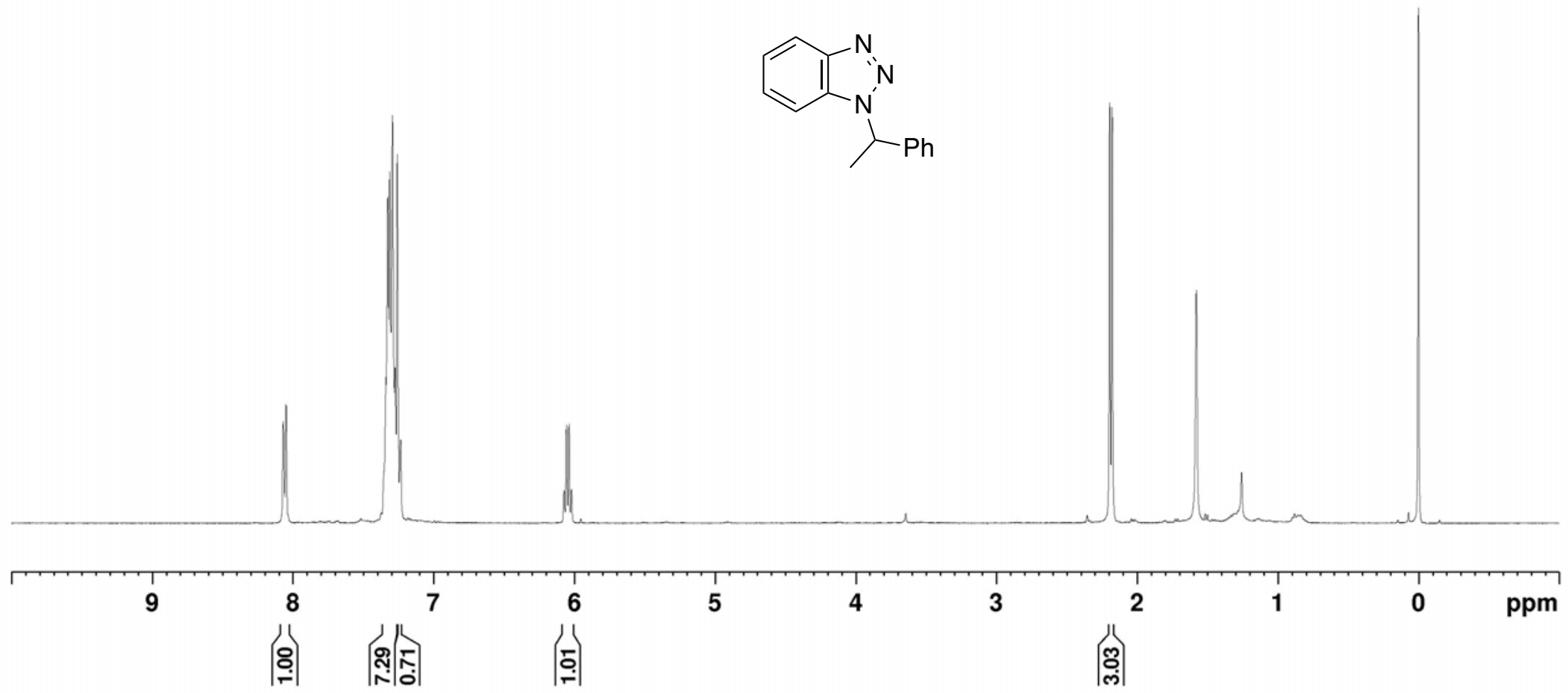

${ }^{1} \mathrm{H}$ NMR spectrum of $\mathbf{3 g m}\left(400 \mathrm{MHz}, \mathrm{CDCl}_{3}\right)$ 


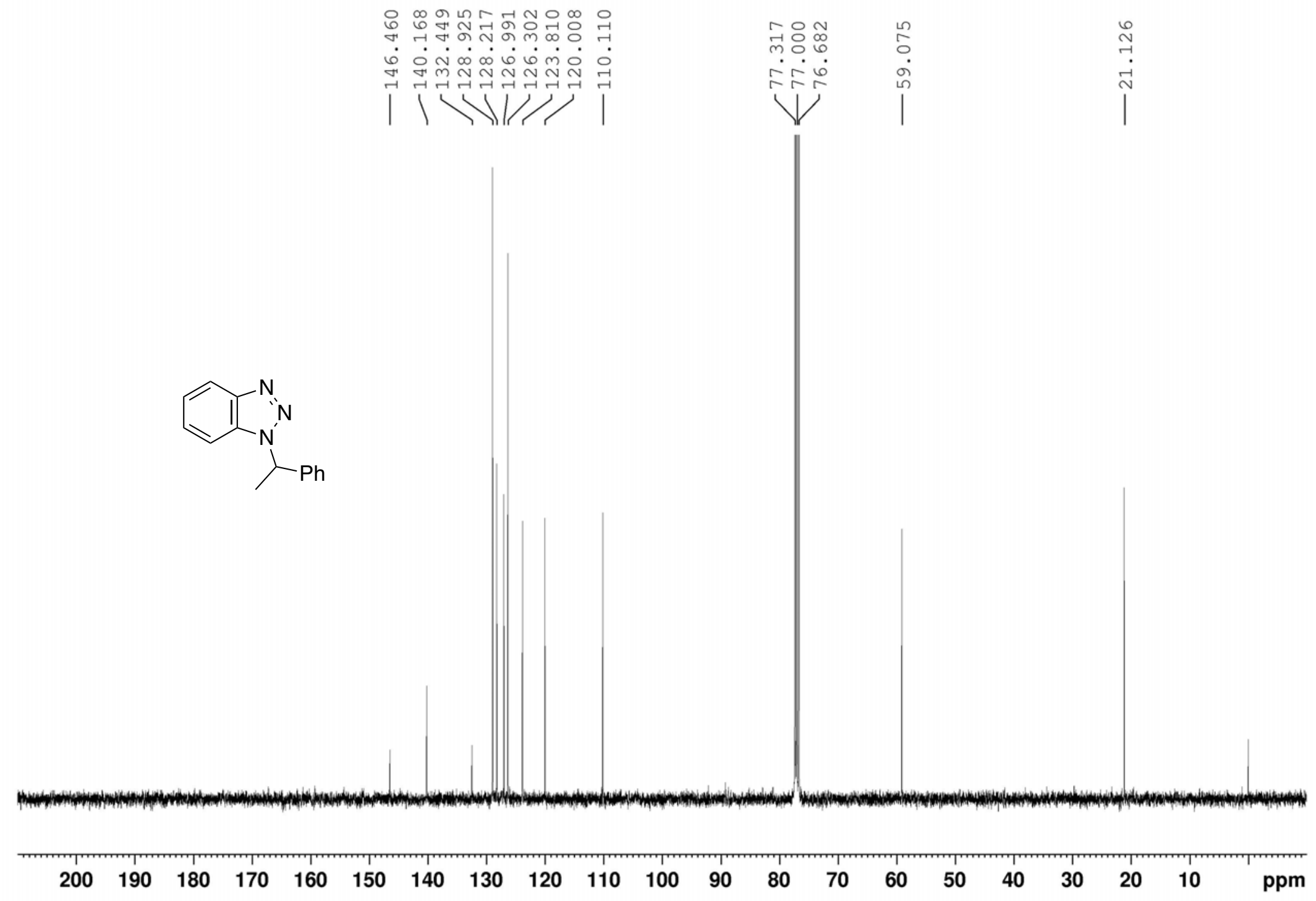

${ }^{13} \mathrm{C}$ NMR spectrum of $\mathbf{3 g m}\left(100.6 \mathrm{MHz}, \mathrm{CDCl}_{3}\right)$ 
东元

.

$\longrightarrow$

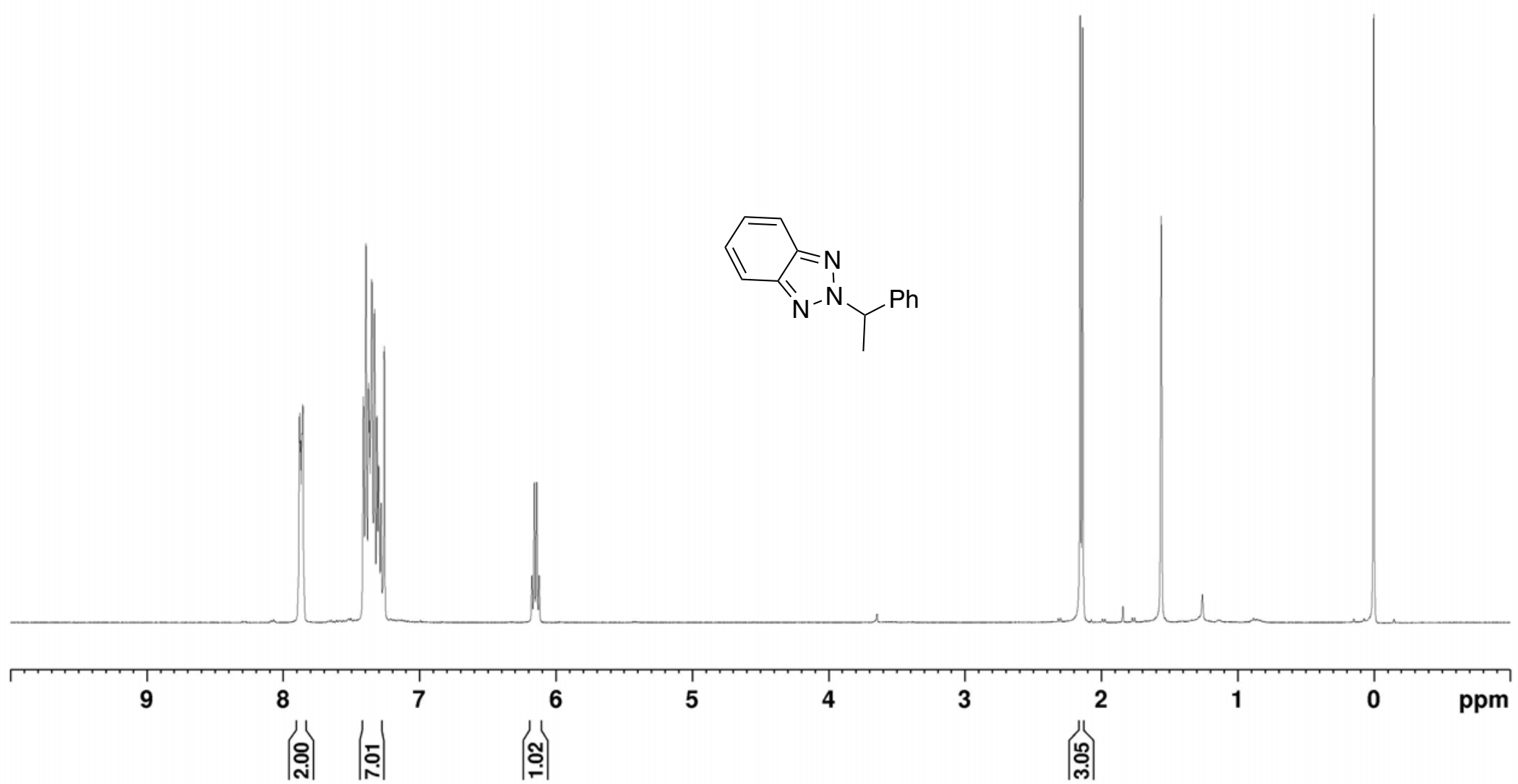

${ }^{1} \mathrm{H}$ NMR spectrum of 3gm' (400 MHz, $\mathrm{CDCl}_{3}$ ) 


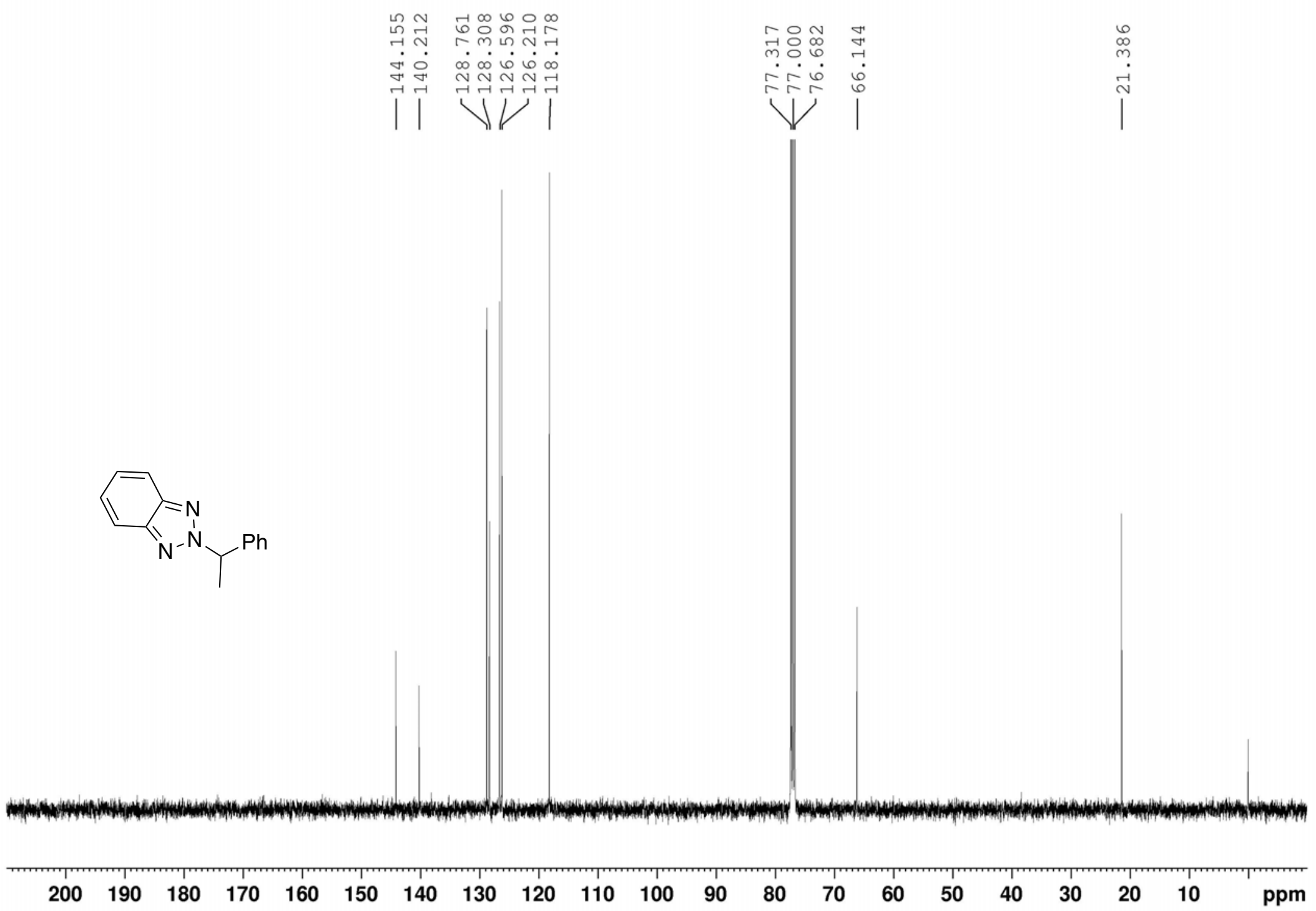

${ }^{13} \mathrm{C}$ NMR spectrum of 3gm' $\left(100.6 \mathrm{MHz}, \mathrm{CDCl}_{3}\right)$ 


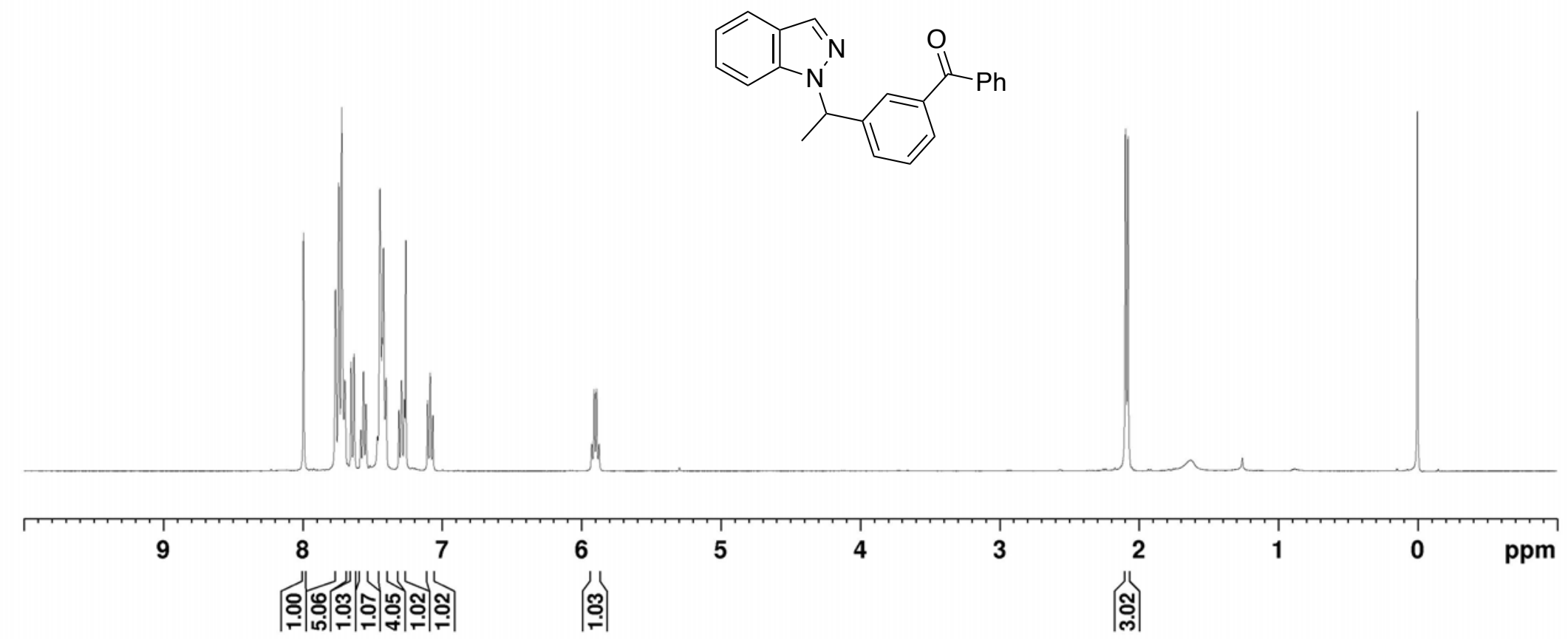

${ }^{1} \mathrm{H}$ NMR spectrum of $\mathbf{3} \mathbf{b n}\left(400 \mathrm{MHz}, \mathrm{CDCl}_{3}\right)$ 


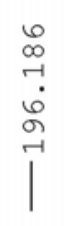

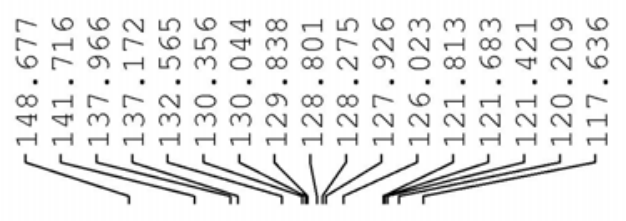<smiles>CC(c1cccc(C(=O)c2ccccc2)c1)n1ncc2ccccc21</smiles>
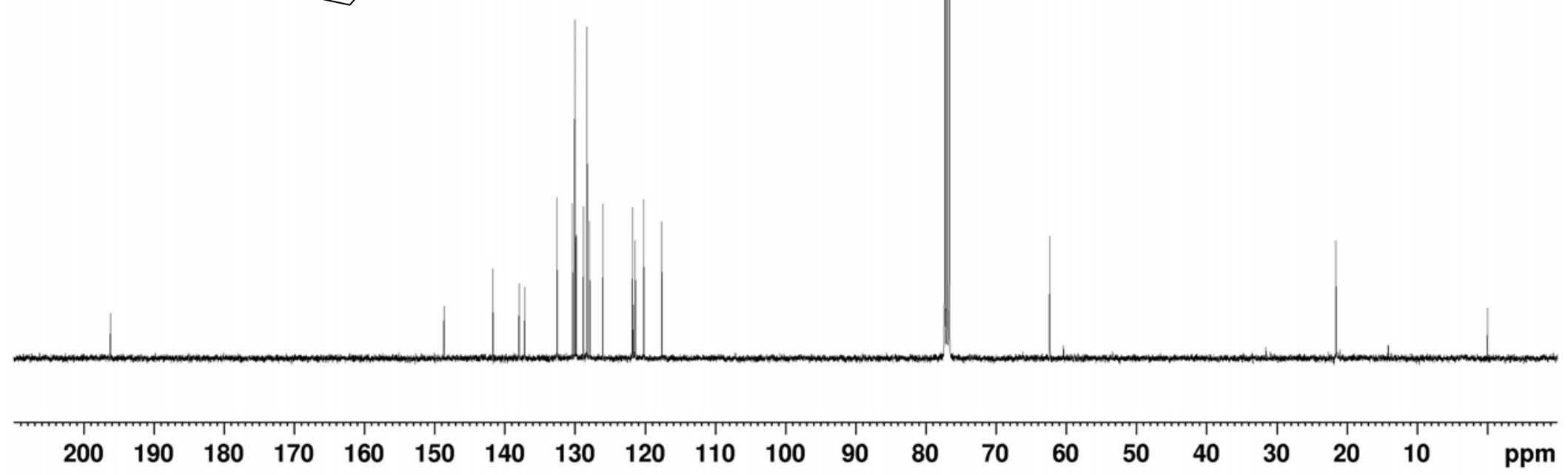

${ }^{13} \mathrm{C}$ NMR spectrum of 3 bn $\left(100.6 \mathrm{MHz}, \mathrm{CDCl}_{3}\right)$

S94 

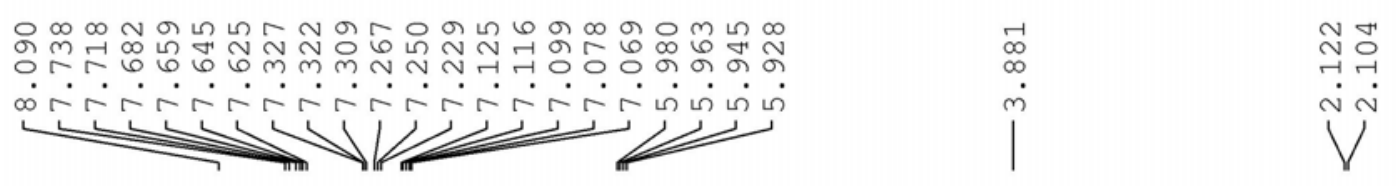

$$
\begin{aligned}
& \circ \\
& \vdots \\
& \vdots \\
& 1
\end{aligned}
$$

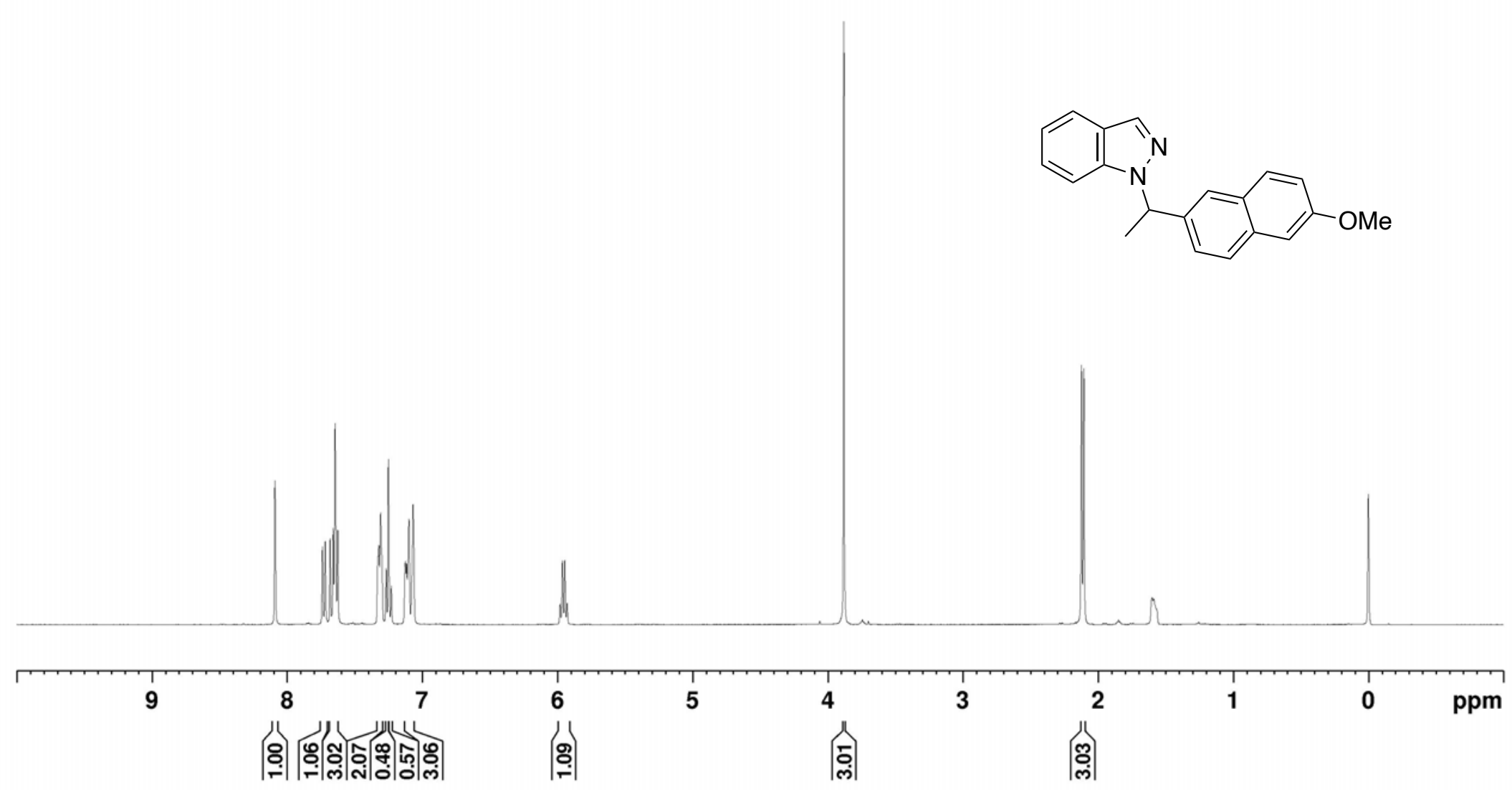

${ }^{1} \mathrm{H}$ NMR spectrum of $\mathbf{3 b o}\left(400 \mathrm{MHz}, \mathrm{CDCl}_{3}\right)$ 
$m$ m

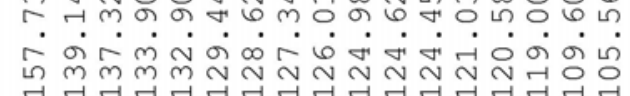

$\mid \underbrace{r+r}$<smiles>COc1ccc2cc(C(C)n3ncc4ccccc43)ccc2c1</smiles>

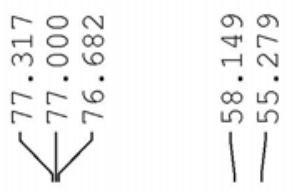

\}

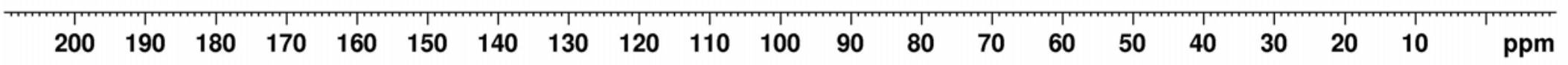

${ }^{13} \mathrm{C}$ NMR spectrum of $3 \mathbf{b o}\left(100.6 \mathrm{MHz}, \mathrm{CDCl}_{3}\right)$

S96 

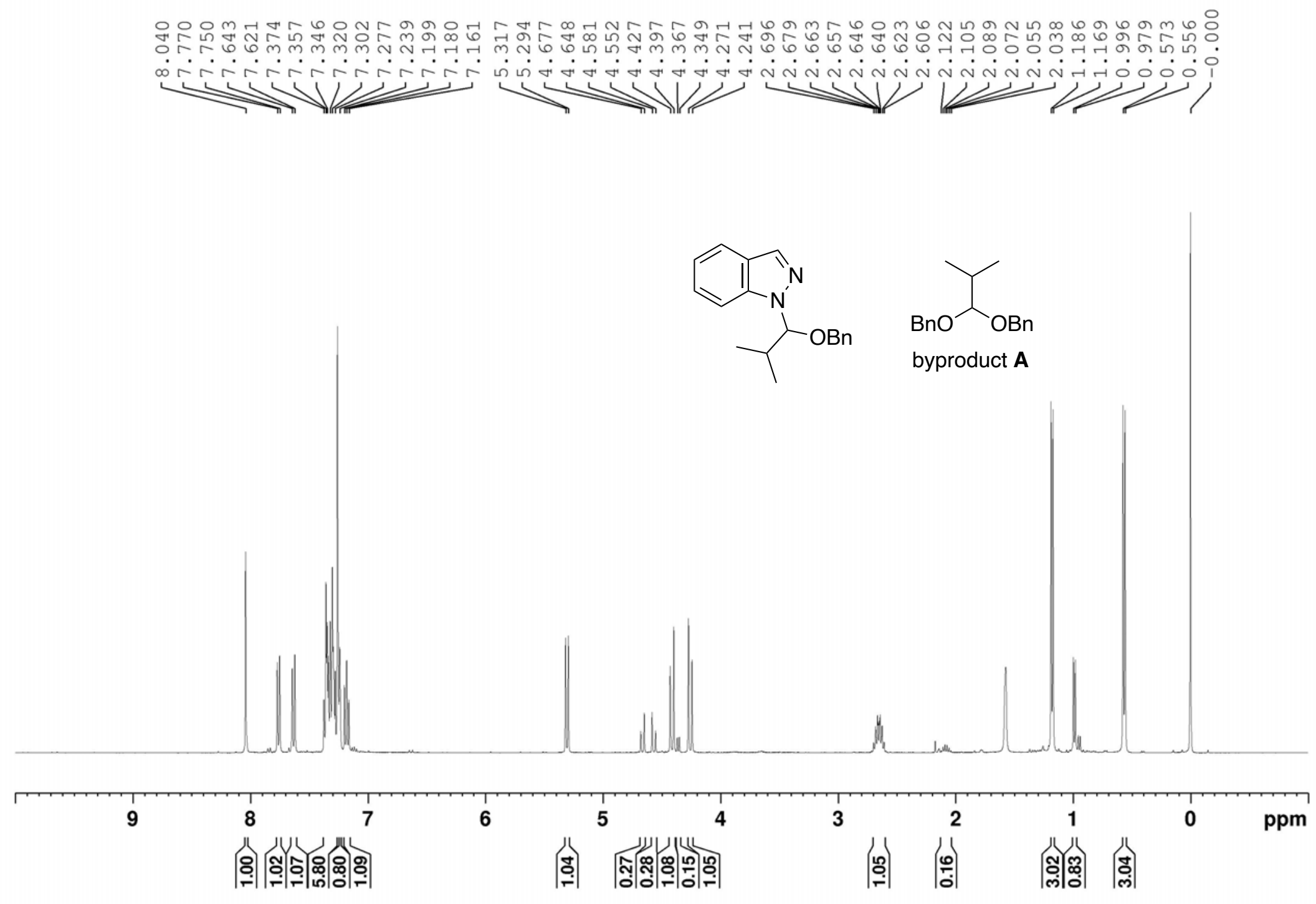

${ }^{1} \mathrm{H}$ NMR spectrum of $\mathbf{3 b p}\left(400 \mathrm{MHz}, \mathrm{CDCl}_{3}\right)$ (The sample contains byproduct $\mathrm{A}$ ) 


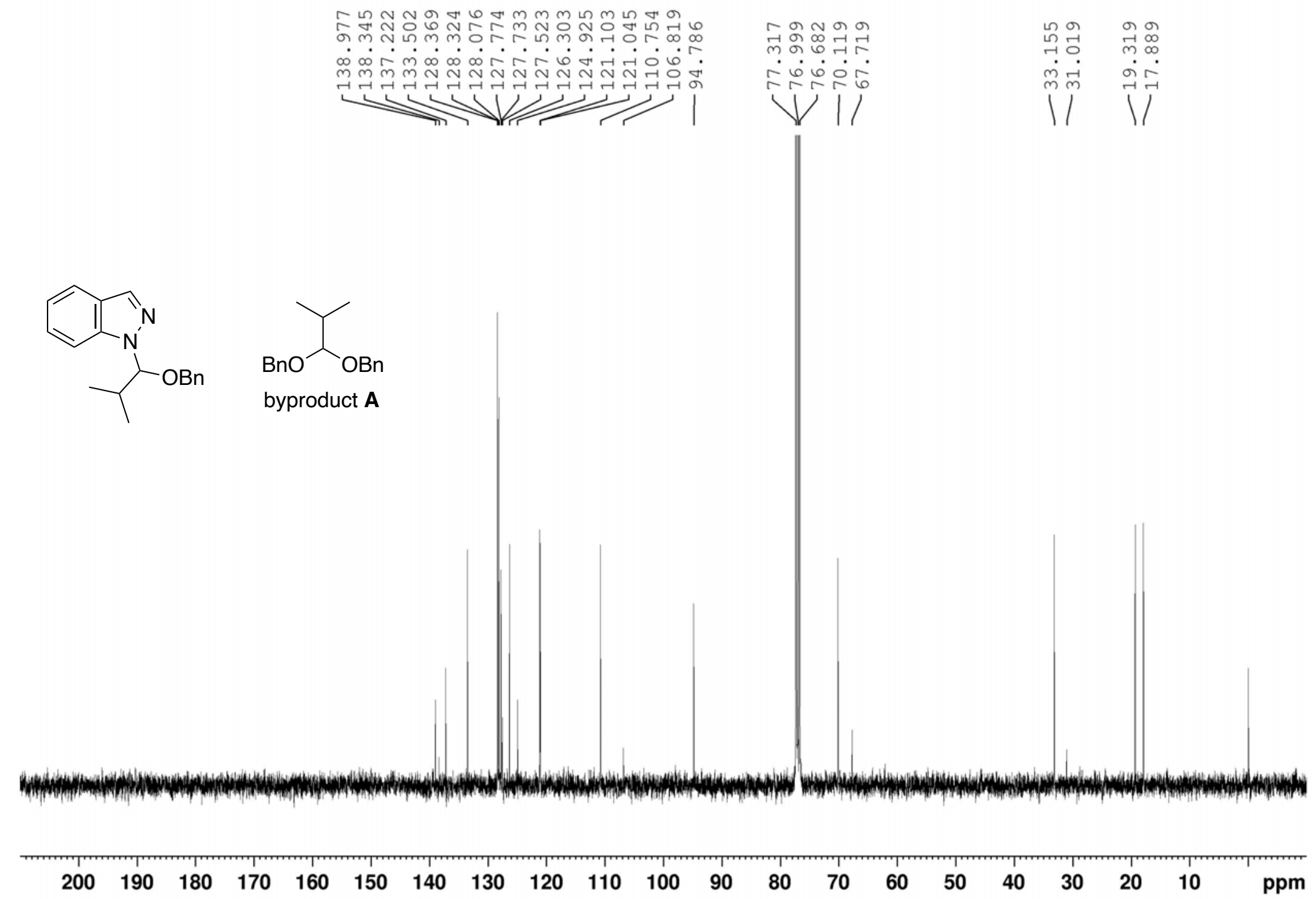

${ }^{13} \mathrm{C}$ NMR spectrum of $\mathbf{3 b p}\left(100.6 \mathrm{MHz}, \mathrm{CDCl}_{3}\right)$ (The sample contains byproduct $\left.\mathrm{A}\right)$ 


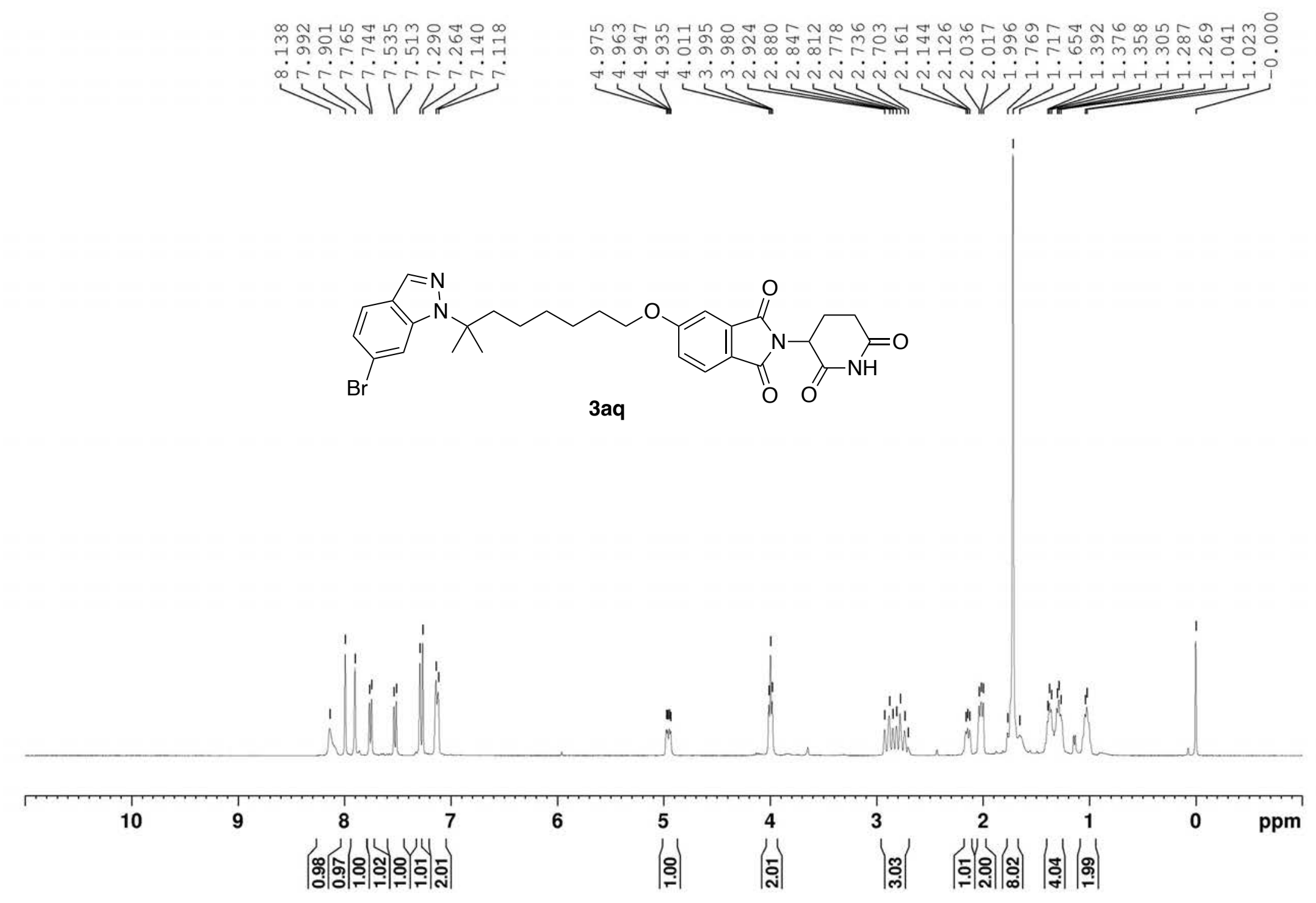

${ }^{1} \mathrm{H}$ NMR spectrum of $\mathbf{3 a q}\left(400 \mathrm{MHz}, \mathrm{CDCl}_{3}\right)$ 


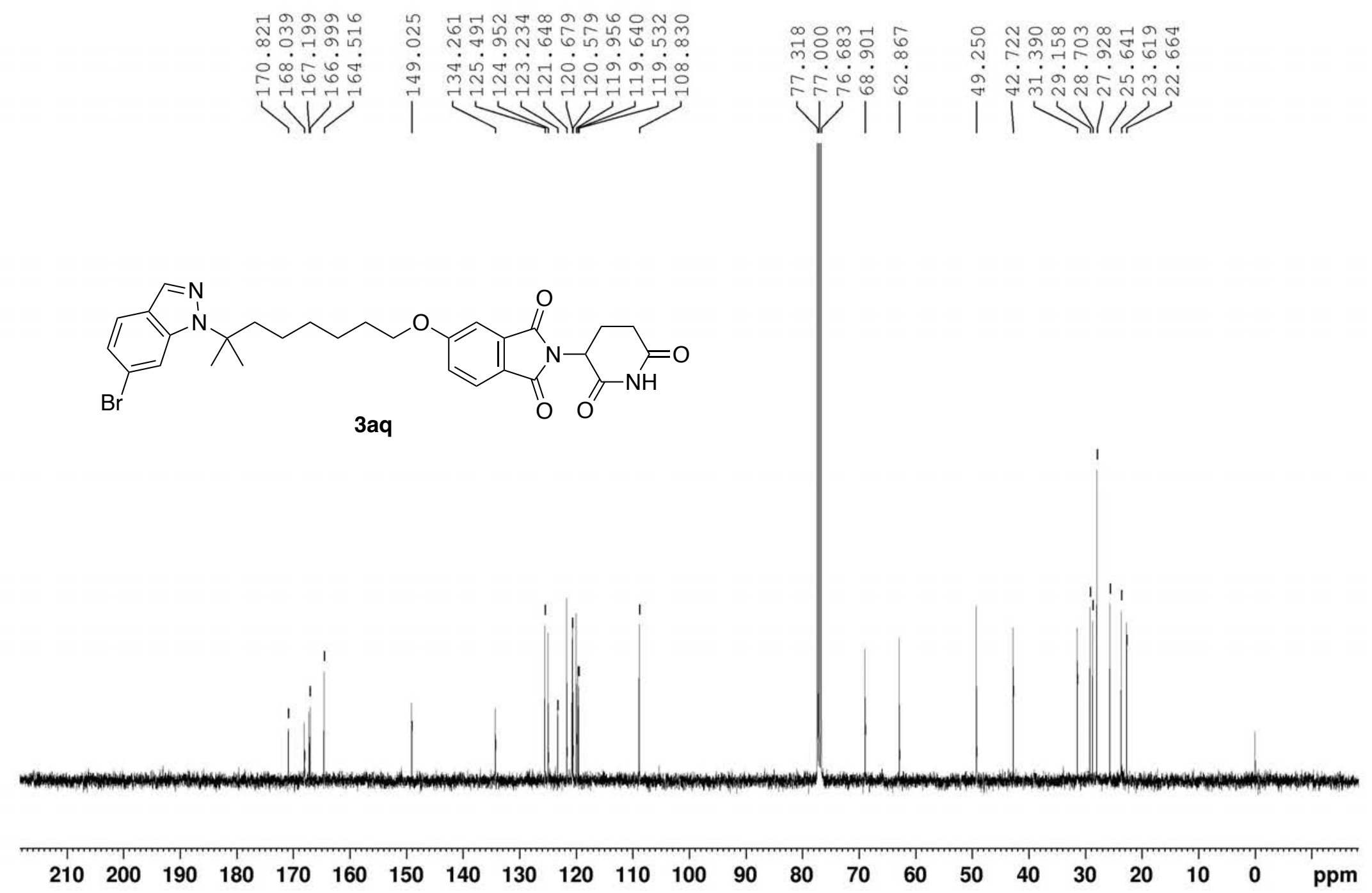

${ }^{13} \mathrm{C}$ NMR spectrum of $\mathbf{3 a q}\left(100.6 \mathrm{MHz}, \mathrm{CDCl}_{3}\right)$ 